\title{
MEETING THE COOL NEIGHBORS. IX. THE LUMINOSITY FUNCTION OF M7-L8 ULTRACOOL DWARFS IN THE FIELD
}

\author{
Kelle L. Cruz, ${ }^{1,2,3,4}$ I. Neill Reid, ${ }^{3,4,5}$ J. Davy Kirkpatrick, ${ }^{6}$ Adam J. Burgasser ${ }^{7}$ James Liebert, ${ }^{3,8}$ \\ Adam R. Solomon, ${ }^{1,9,10}$ Sarah J. Schmidt, ${ }^{1,11,12}$ Peter R. Allen, ${ }^{3,13}$ \\ Suzanne L. Hawley, ${ }^{12}$ and Kevin R. Cover ${ }^{12}$ \\ Received 2006 July 28; accepted 2006 September 23
}

\begin{abstract}
We present a $20 \mathrm{pc}$, volume-limited sample of M7-L8 dwarfs created through spectroscopic follow-up of sources selected from the Two Micron All Sky Survey Second Incremental Release Point Source Catalog. In this paper we present optical spectroscopy of 198 candidate nearby ultracool dwarfs, including 12 late-M and L dwarfs likely to be within $20 \mathrm{pc}$ of the Sun and 94 more distant late-type dwarfs. We have also identified five ultracool dwarfs with spectral signatures of low gravity. Combining these data with previous results, we define a sample of 99 ultracool dwarfs in 91 systems within $20 \mathrm{pc}$. These are used to estimate the $J$ - and $K$-band luminosity functions for dwarfs with optical spectral types between M7 and L8 $\left(10.5<M_{J}<15,9.5<M_{K_{S}}<13\right)$. We find a space density of $4.9 \times 10^{-3} \mathrm{pc}^{-3}$ for late-M dwarfs (M7-M9.5) and a lower limit of $3.8 \times 10^{-3} \mathrm{pc}^{-3}$ for L dwarfs.
\end{abstract}

Key words: Galaxy: stellar content — solar neighborhood — stars: late-type — stars: low-mass, brown dwarfs stars: luminosity function, mass function

Online material: machine-readable tables

\section{INTRODUCTION}

Over the last several years, we have been using the Two Micron All Sky Survey (2MASS; Skrutskie et al. 2006) to carry out a census of late-type dwarfs in the vicinity of the Sun. In particular, we have undertaken the first comprehensive survey of ultracool $\mathrm{M}$ and $\mathrm{L}$ dwarfs in the immediate solar neighborhood (Cruz et al. 2003, [Paper V]). This population of cool, low-luminosity dwarfs includes both very low mass stars and substellar-mass brown dwarfs (Chabrier \& Baraffe 2000; Burrows et al. 2001).

One of the primary aims of our program is the derivation of the luminosity function (the number of objects as a function of absolute magnitude) across the hydrogen-burning limit. The local field luminosity function has been well measured for objects as cool as late-M dwarfs (Reid et al. 2002a), but studies probing to

\footnotetext{
${ }^{1}$ Department of Astrophysics, American Museum of Natural History, New York, NY, USA; kelle@amnh.org.

2 NSF Astronomy and Astrophysics Postdoctoral Fellow.

3 Visiting Astronomer, Kitt Peak National Observatory, National Optical Astronomy Observatory, which is operated by the Association of Universities for Research in Astronomy, Inc., under cooperative agreement with the National Science Foundation.

${ }^{4}$ Visiting Astronomer, Cerro Tololo Inter-American Observatory, National Optical Astronomy Observatory, which is operated by the Association of Universities for Research in Astronomy, Inc., under contract with the National Science Foundation.

5 Space Telescope Science Institute, Baltimore, MD, USA.

6 Infrared Processing and Analysis Center, California Institute of Technology, Pasadena, CA, USA.

7 Massachusetts Institute of Technology, Kavli Institute for Astrophysics and Space Research, Cambridge, MA, USA.

${ }^{8}$ Department of Astronomy and Steward Observatory, University of Arizona, Tucson, AZ, USA.

9 John F. Kennedy High School, Bellmore, NY, USA.

10 Department of Astronomy, Yale University, New Haven, CT, USA.

11 Department of Physics and Astronomy, Barnard College, Columbia University, New York, NY, USA.

12 Department of Astronomy, University of Washington, Seattle, WA, USA.

13 Department of Astronomy and Astrophysics, Pennsylvania State University, University Park, PA, USA.
}

fainter magnitudes have been limited by poor statistics and low sensitivity (Reid et al. 1999; Kirkpatrick et al. 1999, 2000; Gizis et al. 2000; Burgasser 2002). Pushing to lower luminosities and larger volumes is crucial to measuring the contribution of brown dwarfs to the local space density.

The initial results from our program to create a statistically well-defined sample of nearby ultracool dwarfs were presented in Paper V. In that paper we described how we constructed a sample of 630 candidate nearby late-M and L dwarfs from the 2MASS Second Incremental Data Release Point Source Catalog (PSC), designated the 2MU2 sample. We also presented follow-up spectroscopic observations of a subset of the ultracool candidates. In this paper we present the remainder of the far-red optical spectroscopic observations and combine these results with previous observations to construct the $J$ - and $K_{S}$-band luminosity functions. These data include many newly discovered late-type dwarfs, including 12 additions to the core $20 \mathrm{pc} 2 \mathrm{MU} 2$ sample of M7-L8 dwarfs estimated to be within $20 \mathrm{pc}$ and used to measure the luminosity function.

This is the ninth paper in the series and follows directly from Paper V. The first three papers (Reid \& Cruz 2002; Reid et al. 2002b; Cruz \& Reid 2002) and Papers VII and VIII (Reid et al. $2003 b$, 2004) concentrated on proper-motion-selected $\mathrm{K}$ and M dwarfs. Paper IV (Reid et al. 2003a) describes the discovery of 2MASS J18353790+3259545, an M8.5 dwarf within $6 \mathrm{pc}$ of the Sun (which is included in the present analysis). Results from a search for ultracool dwarfs lying close to the Galactic plane are presented in Reid (2003 [Paper VI]).

Here we build on Paper V and present the remainder of the farred optical spectroscopic follow-up of the 2MU2 sample and derive the $J$ - and $K_{S}$-band luminosity functions based on objects estimated to be within $20 \mathrm{pc}$. In $\S 2$ we present an overview of the characteristics of the 2MU2 sample including its creation and follow-up status. Spectroscopic observations from seven telescopes over 16 runs are described in $\S 3$. The results of the spectroscopy and the methods used to estimate spectral types, absolute magnitudes, and distances are in $\S 4$. Interesting individual objects 
are presented in $\S 5$. In $\S 6$ we examine the completeness of the 20 pc 2 MU2 sample and derive the luminosity function. Finally, we discuss these results in $\S 7$ and briefly summarize our findings in $\S 8$.

\section{THE 2MU2 SAMPLE}

The creation of the 2MU2 sample is meticulously discussed in Paper V; however, we briefly summarize the selection criteria and procedures here, making particular note of the minor changes that have since been applied. Eleven million point sources were selected from the 2MASS Second Incremental Data Release PSC with $|b|>10^{\circ},\left(J-K_{S}\right)>1.0$, and $J<16.5$. The sample was narrowed to 1672 objects using optical and near-infrared colorcolor and color-magnitude criteria, ${ }^{14}$ and excluding sources with positional coincidence with star formation regions (e.g., Taurus and Orion), high star density regions (e.g., the Large Magellanic Cloud and M31), and other reddening regions (listed in Paper V, Table 3). Below we discuss the further reduction of the sample to 630 objects and the follow-up status of the 518 candidates requiring additional observations.

\subsection{Narrowing and Refining the Sample}

As discussed in Paper V 588 of the 1672 sources in the 2MU2 sample have $J \leq 9$. Those bright sources were considered separately in the appendix of Paper $V$, and they include no new, nearby late-type dwarfs. Ninety-nine of the remaining 1084 candidates were eliminated based either on previous observations listed in the SIMBAD database ${ }^{15}(24)$, positional coincidence with known interstellar clouds (41), or near-infrared colors consistent with mid-type M dwarfs (34 tagged "bland"). We have eliminated a further 355 stars based on the following criteria:

Visual inspection.-We originally identified 211 sources in Paper V either as artifacts or as being associated with large galaxies or globular clusters. A further eight sources have been added to this list, giving a total of 219 sources eliminated.

$(F-J) /\left(J-K_{S}\right)$.-We cross-referenced the sample against the Hubble Space Telescope Guide Star Catalog, version 2.2 (Morrison et al. 2001), and used the photographic $F$-band (red) magnitudes to eliminate 137 sources as too blue in $(F-J)$. However, one object, 2MASS J07003664+3157266, proved to be matched incorrectly. Observations by Thorstensen \& Kirkpatrick (2003) show that this object is a nearby L dwarf, and it is included in the present sample. We have double-checked the remaining $136(F-J)$ rejectees, paying particular attention to outliers in the $\left[(J-H),\left(H-K_{S}\right)\right]$ plane. No additional ultracool candidates were recovered.

Of the remaining 630 ultracool candidates, 112 objects were already known as nearby, late-type dwarfs at the time of publication of Paper V, thus leaving 518 sources requiring follow-up observations.

\subsection{Follow-up Status}

We presented far-red optical spectroscopy of 298 sources in Paper V; optical and/or near-infrared spectroscopy has since been obtained for the remaining 220 targets. If near-infrared data suggested that a source is an L dwarf within $20 \mathrm{pc}$, additional optical spectroscopy was obtained, since $M_{J}$ of $\mathrm{M}$ and L dwarfs is better correlated with optical spectral types than infrared types.

\footnotetext{
${ }^{14}$ Eq. (1) in Paper V incorrectly stated the slope of the $J,\left(J-K_{S}\right)$ selection criterion as 1.5, when the correct value is 3 . The line is plotted correctly in Fig. 1 of Paper V.

${ }^{15}$ See http://simbad.u-strasbg.fr.
}

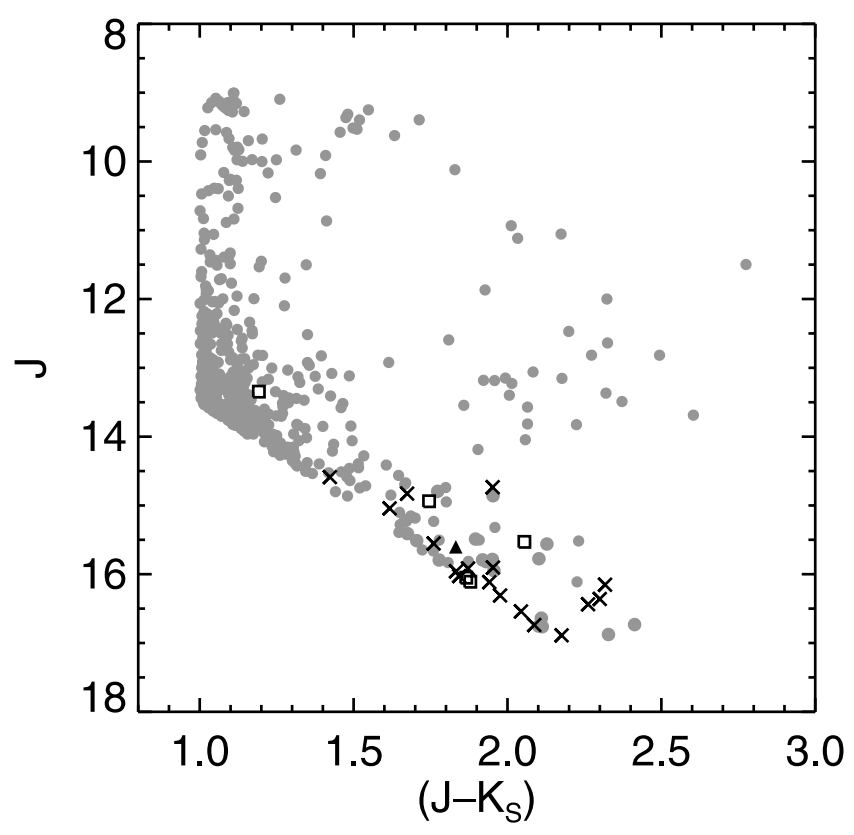

FIG. 1.- Status of the follow-up observations of the 518 candidate ultracool dwarfs in the 2MU2 sample. Far-red optical spectra (circles) have been obtained for 495 objects in the sample. Near-infrared spectra have been used to eliminate 17 fainter targets from the 20 pc 2 MU2 sample (crosses) and to identify one object near the $20 \mathrm{pc}$ distance limit that requires supplemental far-red data to determine its membership (triangle). Five objects have been classified using data from the literature (squares).

Figure 1 summarizes the current status of our observations. Optical spectroscopy has been obtained for 495 objects. Based on near-infrared spectroscopy, we eliminate 17 sources as either extragalactic objects or ultracool dwarfs well beyond our $20 \mathrm{pc}$ distance limit. One source, 2MASSI J0028208+224905, has nearinfrared spectroscopy indicative of possible membership in the 20 pc 2MU2 sample and still requires optical observations. For five objects we list data from the literature: three from Keck (Kirkpatrick et al. 2001b; J. Kirkpatrick et al. 2007b, in preparation) and two from the Sloan Digital Sky Survey (SDSS; Fan et al. 2000; Hawley et al. 2002).

Our infrared spectroscopic observations of ultracool dwarfs will be discussed in a future paper (K. Cruz et al. 2007, in preparation). Here we present far-red optical spectra for 197 sources from the 2MU2 sample that previously lacked sufficient data.

\section{FAR-RED SPECTROSCOPIC OBSERVATIONS}

We have obtained new optical spectra for 198 objects from the 2MU2 sample (including one reobservation of an object originally reported in Paper V) using NOAO facilities and the $3.5 \mathrm{~m}$ Apache Point Observatory (APO) telescope. ${ }^{16}$ The instrumental setups and data reduction techniques used for these data are the same as those described in Paper V. The objects we used as spectral standards are listed in Table 1.

For all observations, Tables 2-6 list the coordinates (as the 2MASS designation) and the near-infrared photometry from the 2MASS Second Incremental Data Release PSC, ${ }^{17}$ date of observation, and telescope used. Several objects were observed on

\footnotetext{
${ }^{16}$ Spectra have been made available from DwarfArchives.org and on request from K. L. C.

17 There are usually small differences between the astrometry and photometry given in the 2MASS Second Release and the values in the final All-Sky Data Release.
} 
TABLE 1

Spectral Standards Used

\begin{tabular}{|c|c|c|c|}
\hline Optical Spectral Type & Object & Telescope & Reference \\
\hline 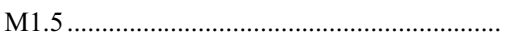 & G1 205 & $\mathrm{KP} 2.1 \mathrm{~m}$ & 1 \\
\hline М2.5 & Gl 250B & CT $1.5 \mathrm{~m}$ & 1 \\
\hline M4.5 & Gl 83.1 & CT $4 \mathrm{~m}$ & 1 \\
\hline 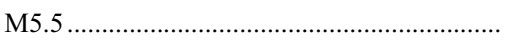 & Gl 65 & CT $4 \mathrm{~m}$ & 1 \\
\hline 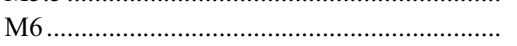 & LHS $1326^{\mathrm{a}}$ & $\mathrm{KP} 2.1 \mathrm{~m}$ & 2 \\
\hline M7 & VB 8 & $\mathrm{KP} 4 \mathrm{~m}$ & 1 \\
\hline 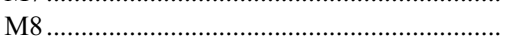 & VB 10 & $\mathrm{KP} 4 \mathrm{~m}$ & 1 \\
\hline 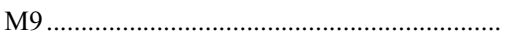 & LHS 2065 & CT $4 \mathrm{~m}$ & 1 \\
\hline L0 & 2MASS J03454316+2540233 & Keck I & 3 \\
\hline L1 „.. & 2MASS J14392837+1929150 & Keck I & 3 \\
\hline 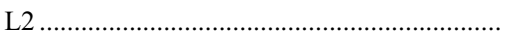 & Kelu 1 & Keck I & 3 \\
\hline 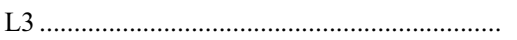 & DENIS-P J1058.7-1548 & Keck I & 3 \\
\hline 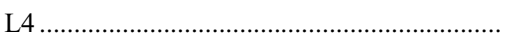 & 2MASS J11550087+2307058 & Keck I & 3 \\
\hline L4.5 & 2MASS J06523073+4710348 & GN & 4 \\
\hline L5 & DENIS-P J1228.2-1547 & Keck I/GS & 3 \\
\hline 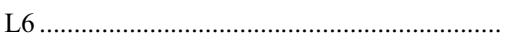 & 2MASS J08503593+1057156 & Keck I & 3 \\
\hline L7 . & DENIS-P J0205.4-1159 & Keck I/GS & 3 \\
\hline L8 & 2MASS J16322911+1904407 & Keck I/GN & 3 \\
\hline
\end{tabular}

NoтE.-While M and L dwarf spectral standards were taken during the course of our observations, we supplemented our data with the publicly available high signal-to-noise ratio Keck I LRIS spectra (DwarfArchives.org).

${ }^{\text {a }}$ Not actually a spectral standard.

References.-(1) Kirkpatrick et al. 1991; (2) Reid et al. 1995; (3) Kirkpatrick et al. 1999; (4) Reid et al. 2007, in preparation.

multiple observing runs to improve the signal-to-noise ratio of the spectra or to monitor for $\mathrm{H} \alpha$ emission-line variability; all observations are listed, and the active objects are noted.

Observations were obtained with the R-C spectrograph on the Kitt Peak $2.1 \mathrm{~m}$ telescope (KP $2.1 \mathrm{~m}$ ) during four runs: 2001 November 1-6, 2002 July 3-8, 2003 March 13-15, and 2003 October 8-12. For all runs, the 400 line $\mathrm{mm}^{-1}$ grating, blazed at $8000 \AA$, was used with the OG 550 order blocking filter to give spectra covering 6000-10000 A. Observations were made using a $1.2^{\prime \prime}-1.5^{\prime \prime}$ slit widths to accommodate various conditions. We obtained an average resolution of $5.8 \AA$ (3.1 pixels). Internal quartz flats and $\mathrm{HeNeAr}$ arcs were taken at each position to correct for fringing for all targets except for the one object observed during 2001 November (2MASSI J0959560+200234), for which calibration frames were taken nightly.

The Double Imaging Spectrograph (DIS II) on the $3.5 \mathrm{~m}$ telescope at APO was used on 2002 April 10, 2002 May 14, 2002 May 30, and 2002 July 10-11. A $1^{\prime \prime}$ wide slit and the mediumresolution grating with 300 line $\mathrm{mm}^{-1}$ on the red camera was used to cover $6000-10000 \AA$ at a resolution of $7.3 \AA$ (2.4 pixels). Conditions were mostly clear with $0.8^{\prime \prime}-1.2^{\prime \prime}$ seeing.

The MARS instrument on the Mayall $4 \mathrm{~m}$ telescope on Kitt Peak (KP 4 m) was used for three runs: 2002 September 25-28, 2003 July 9-14, and 2004 February 10-12. The VG0850-450 grism was used for all three runs with $1.5^{\prime \prime}-2^{\prime \prime}$ slit widths, to cover $6300-10000 \AA$ at a spectral resolution $\sim 10 \AA$ (3.3 pixels). A CCD detector was used in 2002 September that was older than that used in the two subsequent runs. The main difference between the two detectors is the cosmic-ray event rate, which does not affect the spectral data presented here. The conditions in 2003 July were significantly hampered by the combination of a nearly full moon and smoke from the nearby Aspen fire. The conditions were clear for the other runs.

Observations were obtained on 2003 April 20-23 and 2006 January 13-14 with the R-C spectrograph and Loral $3 \mathrm{~K} \mathrm{CCD} \mathrm{on}$ the Cerro Tololo Inter-American Observatory (CTIO) Blanco $4 \mathrm{~m}$ telescope (CT $4 \mathrm{~m}$ ). We used a 1" wide slit, an OG 515 filter to block higher orders, and a 315 line $\mathrm{mm}^{-1}$ grating blazed at $7500 \AA$ to cover the range 5500-10000 $\AA$ with a resolution of $7 \AA$ (3.5 pixels). All six nights were clear, with seeing ranging from $0.5^{\prime \prime}$ to $1.5^{\prime \prime}$.

Data were obtained with the CTIO $1.5 \mathrm{~m}$ (CT $1.5 \mathrm{~m})$ telescope on 2003 November 7-11 with a Loral $1 \mathrm{~K}$ CCD and the R-C spectrograph. A total lunar eclipse on 2003 November 9 enabled the observations of several fainter objects. We employed a $1.5^{\prime \prime}$ wide slit, an OG 530 filter to block higher orders, and a 400 line $\mathrm{mm}^{-1}$ grating blazed at $8000 \AA$ to cover $6300-9000 \AA$ at a resolution of $6.5 \AA$ (3 pixels). Conditions were clear, and the seeing ranged from $0.7^{\prime \prime}$ to $0.9^{\prime \prime}$.

The Gemini Multi-Object Spectrometer (GMOS; Hook et al. 2004) was used on both Gemini North (GN) and South (GS) during queue observations taken during 2004 September-2005 March (program IDs: GN-2004B-Q-10 and GS-2004B-Q-30). The RG610_G0307 filter and R400_G5305 disperser were used on GN, while on GS the RG610_G0331 filter and R400_G5325 disperser were implemented to cover $6000-10000 \AA$ A. On both telescopes the nod and shuffle mode was used with a $0.75^{\prime \prime}$ wide slit to provide good sky subtraction and a resolution of $5.5 \AA$ (4 pixels). In addition, two consecutive observations were taken with different central wavelengths to obtain spectral coverage over the chip gaps.

A comparison between the GN data from semester 2004B and other observations of ultracool dwarfs reveals an inconsistency in the flux calibration. For example, 2M 0025+47 (L4) was observed with GN and, as can be seen in Figure 2, has a significantly steeper spectral slope longward of $8700 \AA$ than the two other L4 dwarfs plotted, both of which were observed with GS. While we are continuing to investigate the source of this problem with the aim of correcting it, our ability to judge spectral type is not hindered since we observed spectral standards with the same observational setup and data reduction procedure. (This sound methodology also enabled us to recognize that it was a systematic problem and not a true property of the new objects observed.) 
TABLE 2

M6-L8 Dwarfs Discovered within 20 pc

\begin{tabular}{|c|c|c|c|c|c|c|c|c|c|c|c|}
\hline \multirow[b]{2}{*}{ 2MASSI DeSIGNATION ${ }^{\mathrm{a}}$} & \multirow[b]{2}{*}{ 2MUCD No. } & \multirow[b]{2}{*}{ Other NAME } & \multicolumn{3}{|c|}{ 2MASS } & \multirow[b]{2}{*}{ Obs. Date (UT) } & \multirow[b]{2}{*}{ Telescope } & \multirow{2}{*}{$\begin{array}{c}\text { Optical } \\
\text { Spectral Type }\end{array}$} & \multirow[b]{2}{*}{$M_{J}$} & \multirow{2}{*}{$\begin{array}{c}d \\
(\mathrm{pc})\end{array}$} & \multirow[b]{2}{*}{ Other ReFs } \\
\hline & & & $J$ & $J-H$ & $J-K_{S}$ & & & & & & \\
\hline $0123112-692138 \ldots \ldots \ldots \ldots \ldots \ldots$ & 13056 & $\ldots$ & 12.335 & 0.619 & 1.034 & $\begin{array}{l}2003 \text { Nov } 8 \\
2006 \text { Jan } 15\end{array}$ & $\begin{array}{l}\text { CT } 1.5 \mathrm{~m} \\
\text { CT } 4 \mathrm{~m}\end{array}$ & M8 & $11.16 \pm 0.18$ & $17.2 \pm 1.4$ & \\
\hline $0138215-732058 \ldots \ldots \ldots \ldots \ldots \ldots$ & 13063 & $\ldots$ & 11.508 & 0.720 & 1.056 & $\begin{array}{l}2003 \text { Nov } 9 \\
2006 \text { Jan } 15\end{array}$ & $\begin{array}{l}\text { CT } 1.5 \mathrm{~m} \\
\text { CT } 4 \mathrm{~m}\end{array}$ & M6 & $10.12 \pm 0.37$ & $19.0 \pm 3.3$ & \\
\hline $0318540-342129$ & 10176 & $\ldots$ & 15.530 & 1.221 & 2.055 & 2005 Oct 10 & GS & L7 & $14.45 \pm 0.30$ & $16.5 \pm 2.3$ & 1 \\
\hline 0340094-672405 ………........... & 10202 & $\ldots$ & 14.740 & 1.172 & 1.799 & 2006 Jan 14 & CT $4 \mathrm{~m}$ & L7:: & $14.45 \pm 0.90$ & $11.4 \pm 4.7$ & \\
\hline 0544115-243301_.................... & 10444 & $\ldots$ & 12.518 & 0.656 & 1.079 & 2002 Sep 25 & KP $4 \mathrm{~m}$ & M8 & $11.16 \pm 0.18$ & $18.7 \pm 1.6$ & \\
\hline $0700366+315726 \ldots \ldots \ldots \ldots \ldots \ldots \ldots$ & 10617 & $\ldots$ & 12.922 & 0.967 & 1.614 & $\begin{array}{l}2003 \text { Oct } 12 \\
2004 \text { Feb } 10\end{array}$ & $\begin{array}{l}\text { KP } 2.1 \mathrm{~m} \\
\mathrm{KP} 4 \mathrm{~m}\end{array}$ & L3.5 comb $^{\text {b }}$ & $12.49 \pm 0.06^{\mathrm{b}}$ & $12.2 \pm 0.3^{\mathrm{b}}$ & 2,3 \\
\hline $1043075+222523 \ldots \ldots \ldots \ldots \ldots \ldots \ldots$ & 10926 & $\ldots$ & 15.947 & 1.202 & 1.956 & 2004 Nov 7 & GN & L8 & $14.77 \pm 0.50$ & $17.2 \pm 4.0$ & \\
\hline $1440229+133923 \ldots \ldots \ldots \ldots \ldots \ldots \ldots$ & 11230 & LSPM J1440+1339 & 12.379 & 0.635 & 1.088 & 2002 Jul 5 & $\mathrm{KP} 2.1 \mathrm{~m}$ & M8 & $11.16 \pm 0.18$ & $17.6 \pm 1.5$ & 4,5 \\
\hline $1534570-141848 \ldots \ldots \ldots \ldots \ldots \ldots . .$. & 11346 & $\cdots$ & 11.390 & 0.659 & 1.079 & $\begin{array}{l}2002 \mathrm{Jul} 4 \\
2006 \mathrm{Jan} 14\end{array}$ & $\begin{array}{l}\text { KP } 2.1 \mathrm{~m} \\
\text { CT } 4 \mathrm{~m}\end{array}$ & M7 & $10.73 \pm 0.25$ & $13.5 \pm 1.6$ & 6 \\
\hline $2002507-052152 \ldots \ldots \ldots \ldots \ldots \ldots$ & 11946 & $\ldots$ & 15.320 & 1.093 & 1.959 & $\begin{array}{l}2002 \text { Sep } 27 \\
2003 \text { Apr } 23\end{array}$ & $\begin{array}{l}\text { KP } 4 \mathrm{~m} \\
\text { CT } 4 \mathrm{~m}\end{array}$ & L6 & $14.02 \pm 0.30$ & $18.2 \pm 2.6$ & \\
\hline $2325453+425148 \ldots \ldots \ldots \ldots \ldots \ldots \ldots$ & 13227 & $\ldots$ & 15.512 & 1.052 & 1.705 & 2004 Sep 20 & GN & L8 & $14.77 \pm 0.30$ & $14.1 \pm 2.0$ & \\
\hline $2351504-253736 \ldots \ldots \ldots \ldots \ldots \ldots . .$. & 12220 & $\ldots$ & 12.458 & 0.731 & 1.170 & 2002 Jul 5 & $\mathrm{KP} 2.1 \mathrm{~m}$ & M8 & $11.16 \pm 0.18$ & $18.2 \pm 1.5$ & \\
\hline
\end{tabular}

Noте.-Table 2 is also available in machine-readable form in the electronic edition of the Astronomical Journal.

a The 2MASS designation is 2MASSI Jhhmmss[.]s \pm ddmmss.

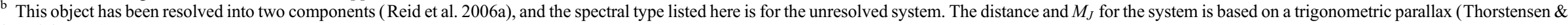
Kirkpatrick 2003).

REFERENCES.-(1) J. Kirkpatrick et al. 2007b, in preparation; (2) Thorstensen \& Kirkpatrick 2003; (3) Reid et al. 2006a; (4) Lépine \& Shara 2005; (5) Lépine 2005; (6) Gizis 2002. 


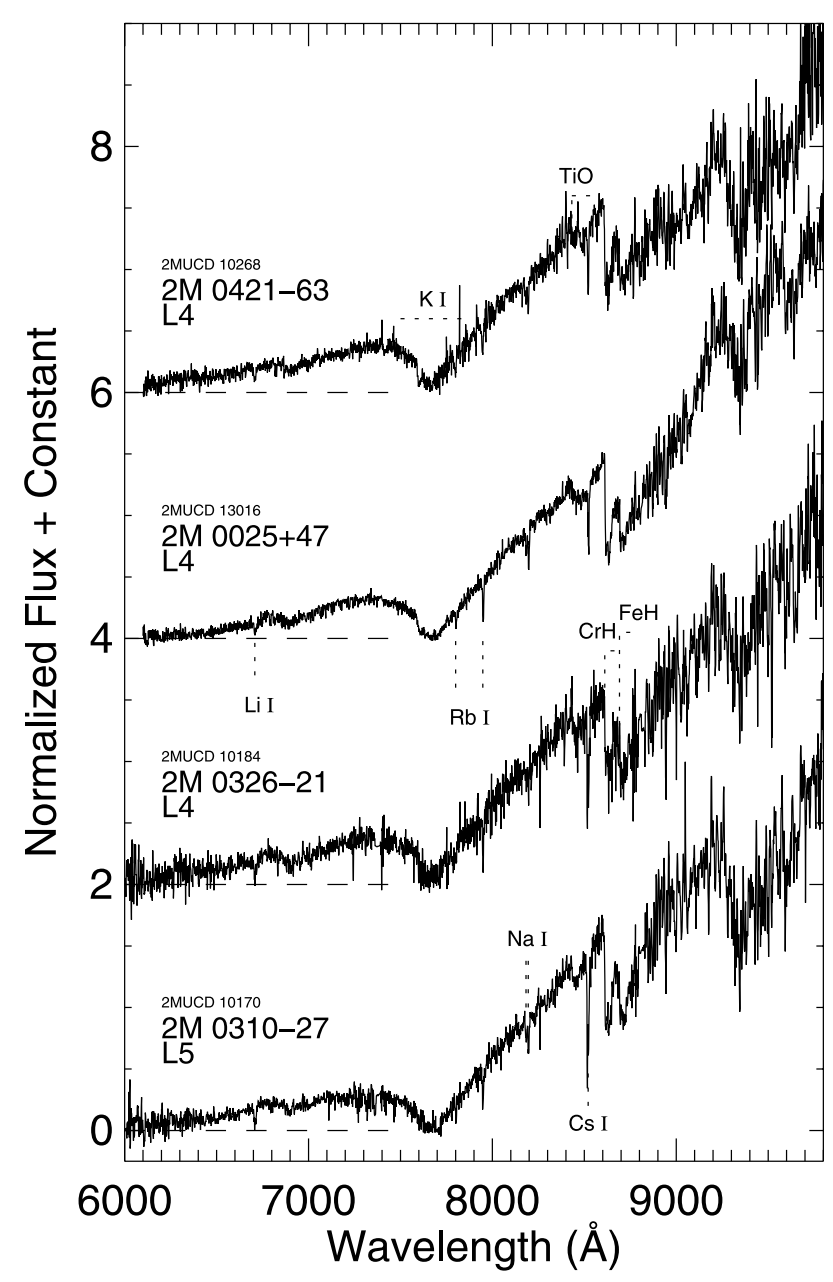

Fig. 2.- Gemini spectra of mid-L dwarfs for which lithium absorption is

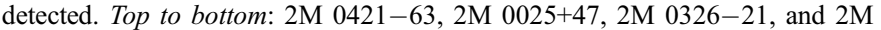
$0310-27$. The steep red slope of $2 \mathrm{M} 0025+47$ is due to a problem with our GN flux calibration. The bottom spectrum is not offset, and the zero points of the offset spectra are shown by dashed lines.

All of the various data were bias subtracted, flat fielded, wavelength calibrated, and flux calibrated using IRAF. ${ }^{18}$ The CCDRED package and the doslit routine were used for the KPNO, CTIO, and APO data, while the Gemini GMOS package was used for the Gemini data.

To wavelength calibrate, measurements of $\mathrm{HeNeAr}$ or $\mathrm{CuAr}$ lamps were taken nightly for most of the observations, at each position for the KP $2.1 \mathrm{~m}$ data and only a few times over the semester for the Gemini queue observations. Flux calibration was done using observations of the flux standards BD $+262606, \mathrm{BD}+17$ 4708, Feige 56, Feige 110, HD 19445, Hiltner 600, G191-B2B, and LTT 2415 (Oke \& Gunn 1983; Massey et al. 1988; Massey \& Gronwall 1990; Hamuy et al. 1994). None of the spectra were corrected for telluric absorption.

\section{RESULTS: SPECTRAL TYPES, MAGNITUDES, AND DISTANCES}

We have measured spectral types, estimated absolute magnitudes, and derived distances for all the observed dwarfs in the

\footnotetext{
${ }^{18}$ IRAF is distributed by the National Optical Astronomy Observatory, which is operated by the Association of Universities for Research in Astronomy, Inc., under cooperative agreement with the National Science Foundation.
}

same manner as described in Paper V. For the late-type dwarfs (spectral types M7 and later), we have assigned a unique, five digit 2MUCD (2MASS Ultracool Dwarf) reference number in addition to the full 2MASS designation. These data are listed in Tables 2-4.

Spectral types are determined via side-by-side comparison with spectra of spectral standards. The spectral standards we used are listed in Table 1. Data for many spectral standards were taken during the course of our observations. ${ }^{19}$ However, we supplemented our data with high signal-to-noise ratio Keck I LRIS data available for $\mathrm{L}$ dwarf spectral standards. ${ }^{20}$

The resulting uncertainty in spectral type is \pm 0.5 subtypes except where low signal-to-noise ratio data result in uncertainties of one or two types, noted in the tables by a single or double colon, respectively. ${ }^{21}$ The spectral types of objects with multiple observations were estimated from the higher signal-to-noise ratio data; typically, this is the second observation, where the spectrum was obtained with a larger aperture telescope than the first.

For objects with spectral types M6 and later, $M_{J}$ is estimated from the spectral-type $/ M_{J}$ calibration derived in Paper V. This $M_{J}$ is combined with photometry from the 2MASS Second Incremental Data Release PSC to yield $M_{K_{S}}$ and spectrophotometric distances. The uncertainties in both the estimated absolute magnitudes and distance are dominated by the uncertainty in the spectral type. Table 2 lists the data for 12 objects with types M6 and later that appear to be within $20 \mathrm{pc}$. Data for 94 more distant late-type objects are listed in Table 3.

Absolute $J$ magnitudes for objects with spectral types earlier than $\mathrm{M} 6$ are estimated using the $\mathrm{TiO}$, $\mathrm{CaH} 2$, and $\mathrm{CaOH}$ spectral indices as described in Cruz \& Reid (2002 [Paper III]). For four objects (2MASSI J0510239-280053, M4; 2MASSI J0544167204909, M5; 2MASSI J1242271+445140, M5; 2MASSI J1436418-153048, M5), at least two of the three spectralindex relations yield two estimates for $M_{J}$. Since these objects fall well outside our distance limit and spectral-type criteria, we have chosen to adopt $M_{J}=8.5 \pm 0.7$ and list the distance as a range. We list data for 34 distant early-to-mid $M$ dwarfs in Table 4.

Not surprisingly, spectroscopy revealed a number of the candidates to be distant giants or carbon stars. Rough spectral types $( \pm 1)$ for the 33 giants were measured via comparison to giant standards (Kirkpatrick et al. 1997; Garcia 1989) and are listed in Table 5. The 17 carbon stars are listed in Table 6.

In Table 7 we list new spectral data for six dwarfs that were presented in Paper V. For five of these we previously listed data from the literature, but we have since reobserved them with our instrumental setup to maintain consistency in the sample. One object, 2MASSI J0326422-210205, was reobserved to obtain a higher signal-to-noise ratio spectrum than that presented in Paper V; these new data revealed a lithium absorption feature (discussed in $\S 5.1$ ). There is general agreement between our new spectral types and those previously quoted.

Five objects with spectral features indicative of low gravity are listed in Table 8 and discussed below in $\S 5.3$. Included among the low-gravity spectra is DENIS J043627.8-411446, which was listed in Paper V with data from the literature, and 2MASSI

\footnotetext{
${ }^{19}$ Data for these and other spectral standards are available from http://research .amnh.org/ kelle/M_standards/.

${ }^{20}$ Available from DwarfArchives.org.

${ }^{21}$ In previous papers in this series, a question mark was used to indicate uncertainty in the spectral type due to a low signal-to-noise spectrum. Here we adopt the more standard notation of a colon.
} 

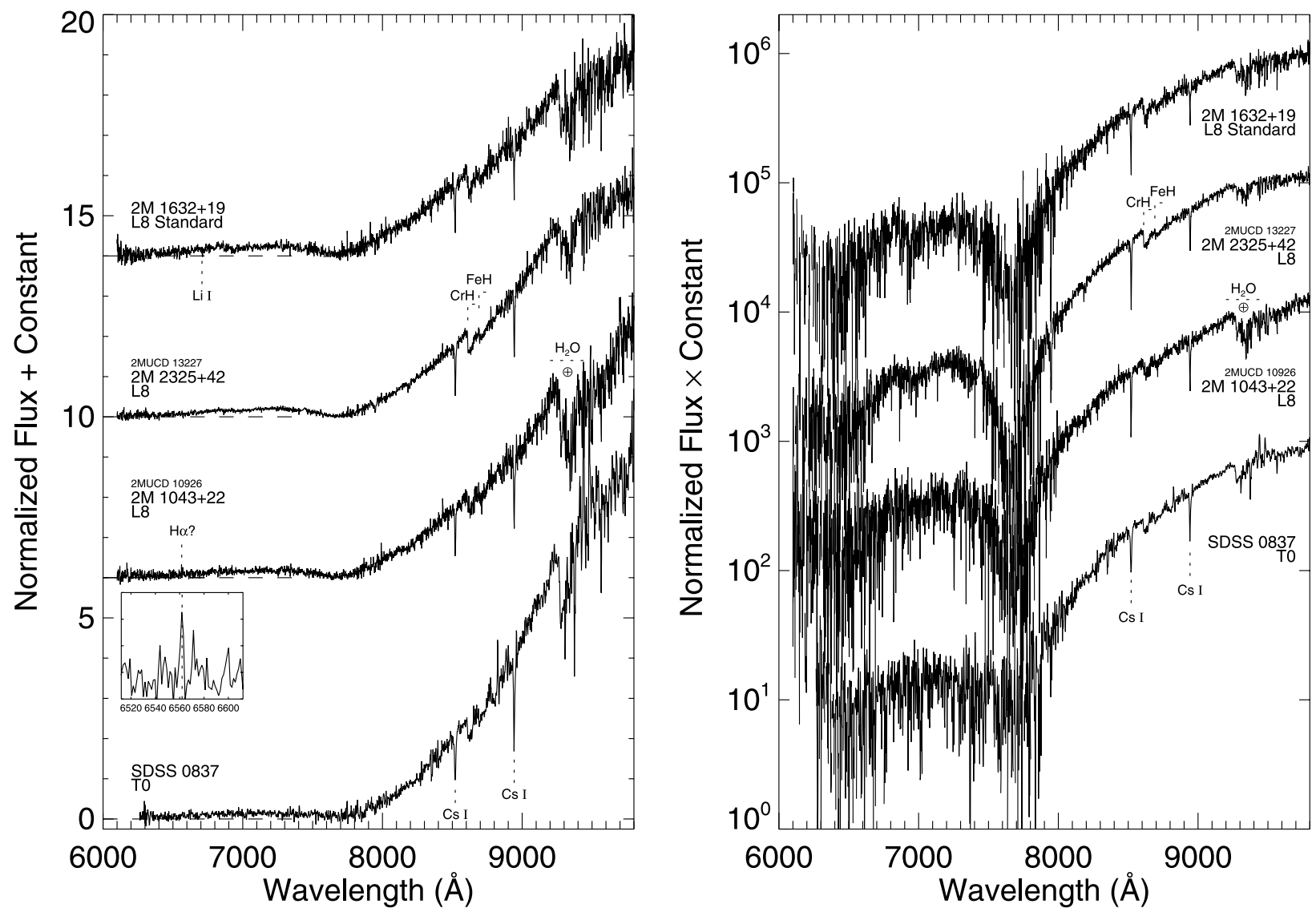

Fig. 3.- Spectra of the two latest-type objects in the present sample, 2M 2325+42 (second from top) and 2M 1043+22 (second from bottom), with the L8 spectral standard 2M 1632+19 (top; Kirkpatrick et al. 1999) and early T dwarf SDSS 0837-00 (bottom; Leggett et al. 2000; Burgasser et al. 2003a; J. Kirkpatrick et al. 2007c, in preparation). The bottom spectrum is not offset, and the zero points of the offset spectra are shown by dashed lines.

J04433761+0002051, which was previously classified as a normal dwarf in Paper V.

\section{INTERESTING INDIVIDUAL OBJECTS}

\subsection{Lithium Detections}

We have detected the Li I absorption line at $6708 \AA$ in six objects in the entire 2MU2 sample. Two of these detections were included in Paper $\mathrm{V}$ and are in the $20 \mathrm{pc}$ sample, 2MASSI J0652307+471034 and 2MASSI J2057540-025230.

Here we present four new detections, although all lie beyond $20 \mathrm{pc}$. Figure 2 displays the spectra of these objects. All four have strong lithium absorption and are spectral type L4-L5: 2MASSI $\mathrm{J} 0025036+475919$, with $\mathrm{EW}=10 \pm 2 \AA$ (also a candidate wide companion; see $\S 5.5$ below), 2MASSI J0421072-630602, with $\mathrm{EW}=6 \pm 2 \AA$, 2MASSI J0310140-275645, with EW $=10 \pm$ $1 \AA$, and 2MASSI J0326422-210205, with EW = $11 \pm 5 \AA$.

The DUSTY theoretical models by Chabrier et al. (2000) suggest that the continued presence of undepleted lithium indicates that these dwarfs are less than 1 Gyr old. Given the absolute magnitudes and effective temperatures inferred from the spectral types, we find that undepleted lithium suggests that these objects are 500 Myr old with masses of $50 M_{\text {Jup }}$.

Our original observations of $2 \mathrm{M} 0326-21,{ }^{22}$ with the CTIO $4 \mathrm{~m}$, lacked sufficient signal-to-noise ratio to permit detection of the

\footnotetext{
22 Source designations in this article are abbreviated in the manner $2 \mathrm{M} \mathrm{hhmm} \pm \mathrm{dd}$, where $2 \mathrm{MASS}$ has been further abbreviated as $2 \mathrm{M}$; the suffix is the sexagesimal right ascension (hours and minutes) and declination (degrees) at J2000.0 equinox.
}

lithium line, and based on those data we estimated a spectral type of L5:. New observations with GS not only reveal lithium absorption but also provide an improved spectral type of L4 (Table 7).

It is noteworthy that all of these new detections were obtained with Gemini $8 \mathrm{~m}$ telescopes. The majority of our observations of L dwarfs were obtained with 4 m class telescopes that are simply not sensitive enough to reliably detect the relatively weak $\mathrm{Li}$ I absorption line.

\subsection{L/T Transition Objects}

The optical spectra of the two latest objects in the new data presented here, 2MASSI J1043075+222523 and 2MASSI J2325453+ 425148 , are shown in Figure 3. The spectrum of $2 \mathrm{M} 2325+42$ is consistent with a spectral type of L8. On the other hand, the red spectrum of $2 \mathrm{M} 1043+22$ is significantly steeper than that of both 2M 2235+42 and the L8 spectral standard. (All three spectra were obtained with the same instrumental setup and data-reduction procedure.)

Infrared spectra show that $2 \mathrm{M} 1043+22$ lacks significant methane absorption in the $H$ band, thus ruling out a T spectral type (K. Cruz et al. 2007, in preparation). As a result, we adopt a spectral type of L8 for 2M 1043+22. We note, however, that it is the reddest L8 dwarf (where the spectral type is based on optical data).

One possible explanation for the unusual spectrum of $2 \mathrm{M} 1043+$ 22 is that it is an unresolved binary similar to SDSS J042348.57041403.5 (Burgasser et al. 2005b) and DENIS-P J225210.7173013 (Reid et al. 2006b). The binary frequency among latetype L dwarfs/early-type T dwarfs has been found to be twice as 


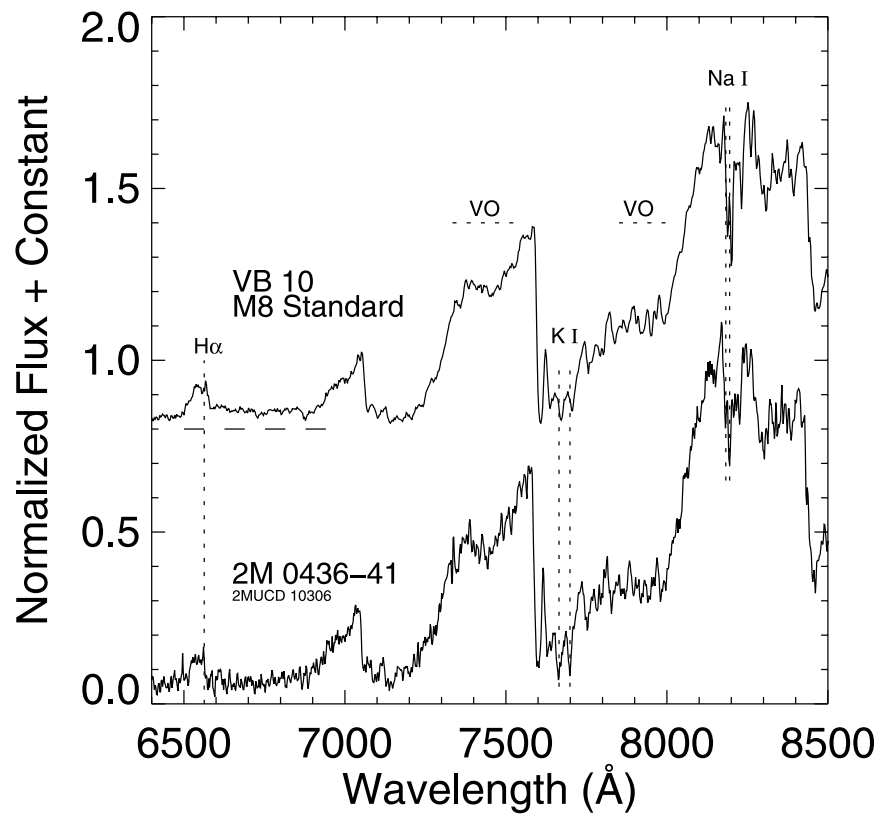

FIG. 4.- Spectrum of the low-gravity dwarf 2M 0436-41 with the M8 standard VB 10. Pressure-/gravity-sensitive features are marked. The bottom spectrum is not offset, and the zero point of VB 10 is shown by a dashed line.

high as that of all other L and T dwarfs (Burgasser et al. 2006b; Liu et al. 2006), and high-resolution imaging observations of 2M 1043+22 are warranted.

In addition, our optical spectrum of $2 \mathrm{M} 1043+22$ shows a feature near the wavelength of $\mathrm{H} \alpha$ that might be consistent with emission. This would be similar to the unusual T dwarfs $2 \mathrm{MASS}$ J10475385+2124234 and SDSS J125453.90-012247.4 found with weak $\mathrm{H} \alpha$ emission by Burgasser et al. (2003a). Further observations are required to verify the reality of the emission in $2 \mathrm{M} 1043+22$.

\subsection{Low-Gravity Objects}

Objects with spectral features indicating low surface gravities $[\log (g)<5$ (cgs units) $]$ are of particular interest, as low gravity implies low mass. In addition, low-mass brown dwarfs with latetype $\mathrm{M}$ and early-type $\mathrm{L}$ spectral types must be younger than their equivalently classified higher mass counterparts. Thus, low gravity suggests both low mass and youth. A growing number of latetype dwarfs with low-gravity features are now being uncovered in field samples (Gizis 2002; Kirkpatrick et al. 2006; J. Kirkpatrick et al. $2007 \mathrm{~b}$, in preparation), and their study is emerging as a new way to probe the evolutionary properties of brown dwarfs and to study their age and mass distributions.

Through comparison to both late-type giants and objects found in young clusters, several gravity-sensitive spectral features have been identified. In particular, we have used enhanced VO bands (7330-7530, 7850-8000 $⿱$ ) , the less broad K I doublet (7665, $7699 \AA)$, and the weaker Na I doublet $(8183,8195 \AA)$ as diagnostics (Martín et al. 1996; Gizis et al. 1999; Gorlova et al. 2003; McGovern et al. 2004; Kirkpatrick et al. 2006).

Five objects with these low-gravity features were presented in Paper V, and we present an additional five here. They are listed along with their proper motions in Table 8 , and their spectra are displayed in Figures 4-7.

The spectrum of 2MASSI J0443376+000205 most resembles an M9, and using this spectral type results in a distance estimate within 20 pc. Even though this object has low-gravity features,

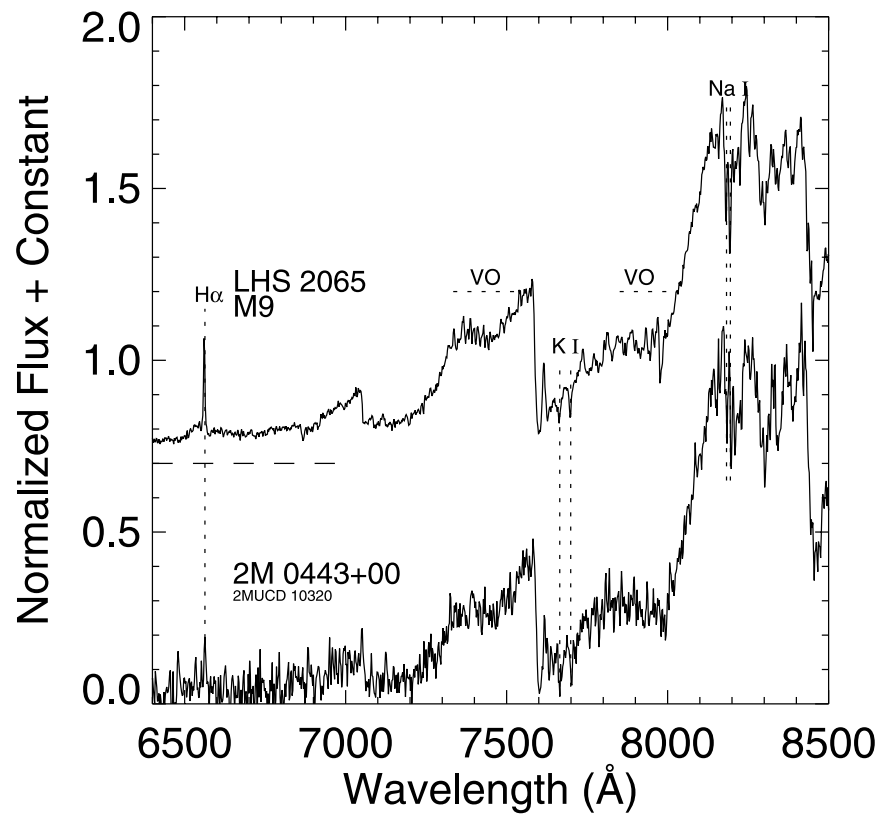

FIG. 5.- Spectrum of the low-gravity dwarf $2 \mathrm{M} 0443+00$ with the M9 standard LHS 2065. Pressure-/gravity-sensitive features are marked. The bottom spectrum is not offset, and the zero point of LHS 2065 is shown by a dashed line.

we include it in the sample used to measure the luminosity function.

The spectra of the two objects in Figure 6, 2MASSI J0241115032658 and 2MASSI J2213449-213607, are later than any of the low-gravity objects from Paper V. While noisy, these spectra are comparable to the spectrum of 2MASS J01415823-4633574, recently described by Kirkpatrick et al. (2006) and estimated to have an age of $1-50 \mathrm{Myr}$ and a mass of $6-25 M_{\text {Jup }}$. All three objects are most comparable to an L1 dwarf but exhibit strong VO molecular

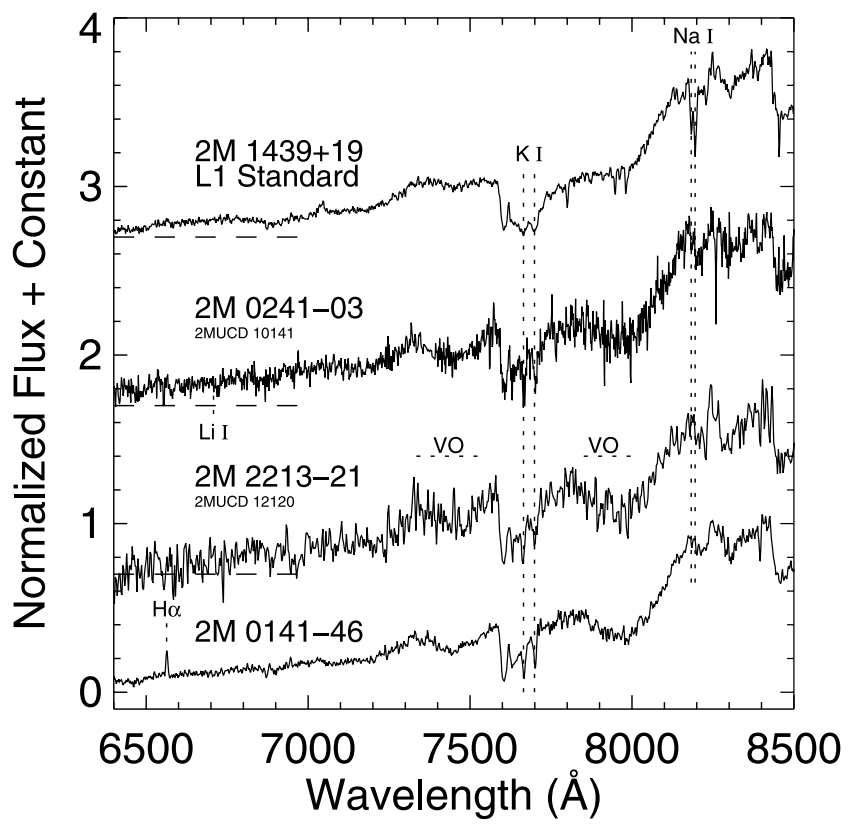

FIG. 6.- Spectrum of the low-gravity dwarfs 2M 0241-03 (second from top) and 2M 2213-21 (second from bottom), with the L1 standard 2M 1439+19 (top; Kirkpatrick et al. 1999) and the young field dwarf 2M 0141-46 (bottom; Kirkpatrick et al. 2006). Pressure-/gravity-sensitive features are marked. The locations of the $\mathrm{H} \alpha$ emission line and $\mathrm{Li}$ I absorption line are labeled, but these features are not present in all spectra. The bottom spectrum is not offset, and the zero points of the offset spectra are shown by dashed lines. 


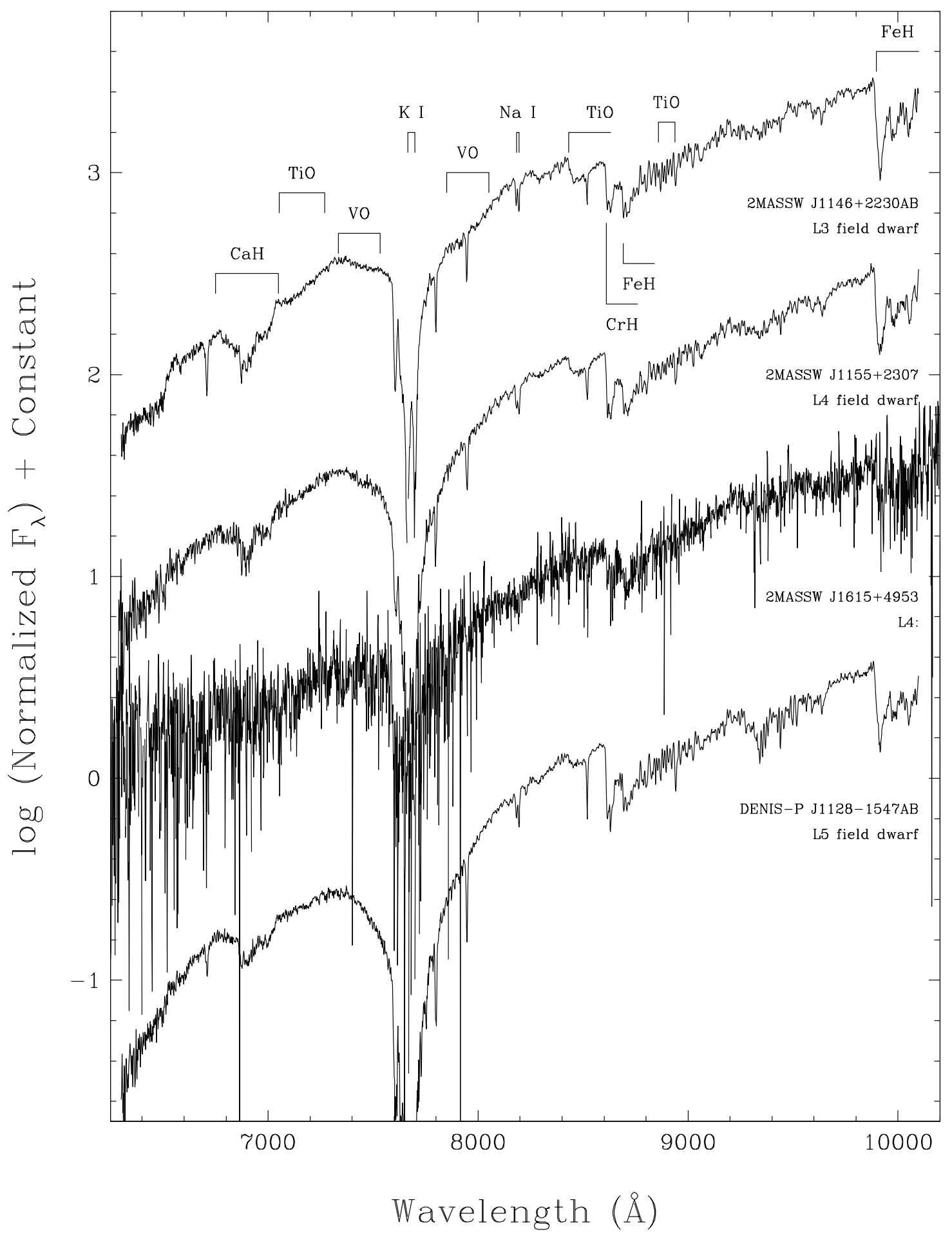

FIG. 7.- Spectrum of the low-gravity dwarf 2M 1615+49 compared to spectral standards of types L3, L4, and L5 from Kirkpatrick et al. (1999). The flux of each spectrum has been normalized to unity at $8250 \AA$, and constant offsets have been added.

absorption and weak $\mathrm{K}$ I and $\mathrm{Na}$ I absorption lines. Unlike 2M 0141$46, \mathrm{H} \alpha$ emission is not detected in either of our new objects.

The spectrum of 2MASSI J1615425+495321 most resembles an L4 dwarf (Fig. 7), but despite the poorer signal of the spectrum departures from the spectral signatures of normal (old) field dwarfs are evident. In particular, the hydride bands of $\mathrm{CaH}, \mathrm{CrH}$, and $\mathrm{FeH}$ are much weaker, as are the core and wings of the normally strong $\mathrm{K}$ I doublet. The bluer slope at the shortest wavelengths can likewise be attributed to weaker absorption by the normally broad $\mathrm{Na}$ I $\mathrm{D}$ doublet located off the short-wavelength end of the plot.
Weaker alkali lines and hydride bands are hallmarks of lower gravity, leading us to believe that this is a young field $\mathrm{L}$ dwarf.

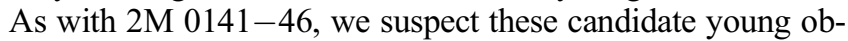
jects to be possible members of the nearby young associations such as the Tucana/Horologium association or the $\beta$ Pic moving group. We are currently in the process of measuring their $U V W$ space motions to definitively test for membership; proper motions are already in hand, and radial velocity measurements of the brighter candidates are underway. In addition, these objects are the focus of highresolution imaging programs searching for even fainter companions. 


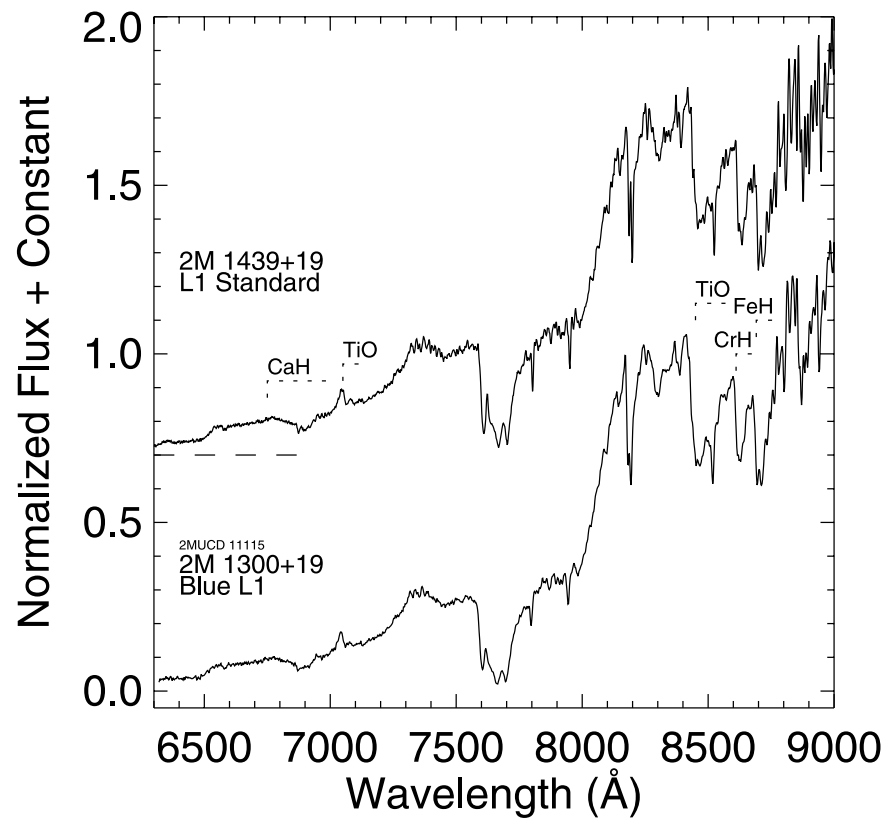

FIG. 8. - Spectrum of blue L1 dwarf $2 \mathrm{M} 1300+19$ with the L1 standard 2M 1439+19 (Kirkpatrick et al. 1999). Metallicity-sensitive spectral features are marked. Despite blue near-infrared colors and fast kinematics, the spectrum of $2 \mathrm{M} \mathrm{1300+19} \mathrm{does} \mathrm{not} \mathrm{exhibit} \mathrm{any} \mathrm{unusual} \mathrm{features.} \mathrm{The} \mathrm{bottom} \mathrm{spectrum} \mathrm{is} \mathrm{not}$ offset, and the zero point of the spectral standard is shown by a dashed line.

\subsection{Two Unusually Blue L Dwarfs: Metal-poor?}

We have identified two objects in the 20 pc 2MU2 sample (included in Table 9 and Figs. 8 and 9) that have unusually blue colors for their spectral type: 2MASSI J1300425+191235 (L1) and 2MASSI J1721039+334415 (L3). We first pointed out these objects in Paper V. The $\left(J-K_{S}\right)$ colors for these two objects are 0.3 and 0.6 mag bluer, respectively, than the mean for their spectral types (Kirkpatrick et al. 2000), a significant deviation suggesting unusual atmospheric properties.

In L dwarfs, variations in $\left(J-K_{S}\right)$ color for a given $T_{\text {eff }}$ can be linked to differences in condensate opacity and metallicity. Condensates are largely responsible for the red near-infrared colors of these objects, with the collective dust particles acting as a warm pseudoblackbody source that radiates predominately at near- and mid-infrared wavelengths (Ackerman \& Marley 2001). A reduction in the condensates in the photosphere, due perhaps to more efficient sedimentation to lower layers in the atmosphere, can lead to bluer near-infrared colors (see Marley et al. 2002, Fig. 1). Knapp et al. (2004) and Chiu et al. (2006) suggested this scenario for seven blue L dwarfs identified in the Sloan Digital Sky Survey.

As one of the brightest L dwarfs, 2M 1300+19 has been moderately well studied. Gelino et al. (2002) and Maiti et al. (2005) both found nonperiodic variability, with the former study suggesting that such variability is evidence for an atmospheric event such as the creation or dissipation of a large storm. If the condensate cloud layer on this object is optically thinner (fewer condensates), then variations in cloud coverage may be more readily detectable. McLean et al. (2003) acquired a $J$-band spectrum of $2 \mathrm{M} 1300+19$ and noted that this object has "the highest equivalent widths in K I of any L1, or indeed almost any other L type." As the $J$ band is highly sensitive to condensate opacity in L dwarfs (Ackerman \& Marley 2001), a reduction in overall condensates may explain the greater optical depth of this line.

A second possibility is reduced atmospheric metallicity, a characteristic of low-mass subdwarfs. With fewer metal species, most molecular opacity is diminished with the exception of $\mathrm{H}_{2}$ absorp-

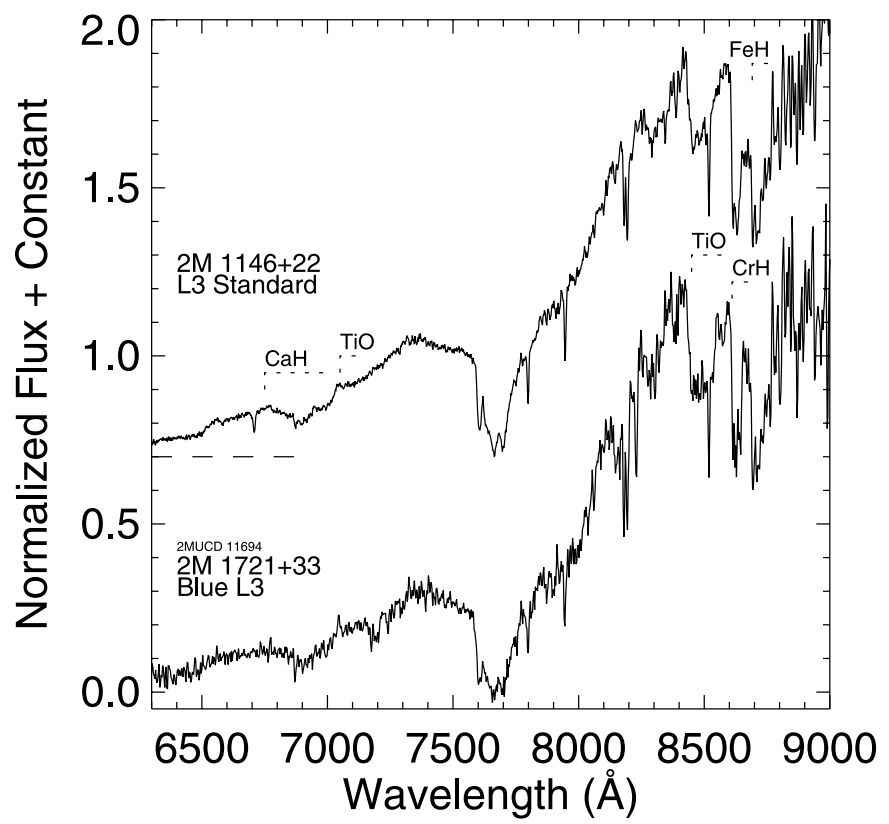

FIG. 9.- Spectrum of blue L3 dwarf $2 \mathrm{M} 1721+33$ with the L3 standard 2M 1146+22 (Kirkpatrick et al. 1999). Metallicity-sensitive spectral features are marked. Despite blue near-infrared colors and fast kinematics, the spectrum of

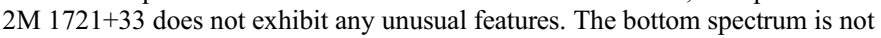
offset, and the zero point of the spectral standard is shown by a dashed line.

tion at $K_{S}$ band. The combination of weaker opacity from metal molecules and enhanced $\mathrm{H}_{2}$ leads to bluer $\left(J-K_{S}\right)$ colors (Linsky 1969; Saumon et al. 1994; Borysow et al. 1997).

Two metal-poor L subdwarfs have already been identified in 2MASS data, 2MASS J05325346+8246465 (Burgasser et al. 2003b) and 2MASS J16262034+3925190 (Burgasser 2004a), and both are quite blue, $(J-K)=0.26$ and -0.03 , respectively. ${ }^{23}$ The redder colors of $2 \mathrm{M} 1300+19$ and $2 \mathrm{M} 1721+33$ suggest that they are not as metal-poor as these L subdwarfs, and their optical spectra do not exhibit the stronger metal-hydride bands $(6750 \AA$ $\mathrm{CaH}, 8611 \AA \mathrm{CrH}, 8692 \AA \mathrm{FeH}$ ) and weaker metal oxides bands (7053, $8432 \AA \mathrm{TiO})$ expected for metal-poor ultracool dwarfs (Burgasser et al. 2006a). Nevertheless, the possibility remains that these objects could be "mild" subdwarfs, with sufficient metal deficiency to enhance $\mathrm{H}_{2}$ absorption.

In addition to being outliers in color space, 2M 1300+19 and $2 \mathrm{M} 1721+33$ stand out kinematically. By combining our spectrophotometric distance with proper motions, we have been investigating the kinematics of the 20 pc $2 \mathrm{MU} 2$ sample (Schmidt et al. 2006). These two objects have two of the three highest tangential velocities in the entire sample: $V_{\tan }=139 \pm 15$ and $98 \pm$ $8 \mathrm{~km} \mathrm{~s}^{-1}$ for $2 \mathrm{M} 1721+33$ and $2 \mathrm{M} 1300+19$, respectively. (The second fastest is 2MASSI J0251148-035245 with $V_{\text {tan }}=125 \pm$ $13 \mathrm{~km} \mathrm{~s}^{-1}$.) These kinematics suggest thick disk membership, which would be consistent with slight metal deficiency $([\mathrm{Fe} / \mathrm{H}] \approx$ $-0.5)$.

The blue colors of these peculiar L dwarfs may also result from a combination of slight metal deficiency and reduced condensate formation. Burgasser et al. (2003b, 2006a) found evidence that condensate formation was inhibited in L subdwarf atmospheres due to the persistence of $\mathrm{TiO}$ molecular bands and $\mathrm{Ti}$ I and $\mathrm{Ca}$ I lines in their optical spectra. These species are generally

\footnotetext{
${ }^{23}$ LSR 1610-0040 was identified as an L subdwarf by Lépine et al. (2003a), but recent work has concluded that it is more likely to be a metal-poor M6 dwarf (Cushing \& Vacca 2006; Reiners \& Basri 2006) or a peculiar binary (A. Burgasser 2006, private communication).
} 
absent in L dwarf spectra due to their incorporation into condensates. While we find no evidence for these features in the spec-

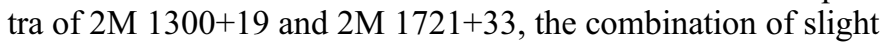
metal deficiency and somewhat reduced condensate formation may tip the near-infrared colors of these objects significantly blueward. Because metallicity significantly impacts the composition and chemistry of low-temperature atmospheres, identifying and studying metallicity effects in blue L dwarfs provide critical empirical constraints for the next generation of theoretical atmosphere models.

\subsection{Candidate Wide Ultracool Companions}

Low-mass stars and brown dwarfs found as wide companions to higher mass main-sequence stars provide opportunities to test evolutionary and atmospheric models since the objects are assumed to be coeval and thus have the same age and metallicity. We have uncovered three ultracool dwarfs, included in Table 3, that are likely wide companions to higher mass stars. These discoveries and others will be discussed by A. Solomon et al. (2007, in preparation).

HD 225118 and 2MASSI J0003422-282241.- These G8 and M7.5 stars constitute a common proper-motion pair separated by $1.1^{\prime}(1700-2600 \mathrm{AU})$. We measured the proper motion of the

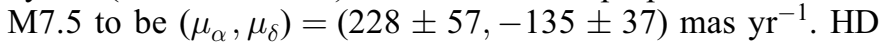
225118 was observed by Hipparcos (Perryman et al. 1997) and was found to have $\left(\mu_{\alpha}, \mu_{\delta}\right)=(280 \pm 1.2,-143 \pm 0.73)$ mas $\mathrm{yr}^{-1}$; in good agreement with the motion of the ultracool dwarf. The parallax of HD 225118 measured by Hipparcos yields a distance of $39.5 \pm 1.7 \mathrm{pc}$. Adopting this distance for the ultracool dwarf yields $M_{J}=10.1 \pm 0.1$, nearly $1 \mathrm{mag}$ brighter than what is expected for an M7.5. Thus, we suspect that the wide ultracool companion might be an unresolved binary itself. An M7- or M8-type companion would bring the spectrophotometric distance into agreement with the Hipparcos distance estimate. If resolved, this would further support the higher binary fraction among widely separated companions found by Burgasser et al. (2005a). For HD 225118, Nordström et al. (2004) estimated a mass of $\sim 0.9 M_{\odot}$, and the age is not well constrained (upper limit of $15.8 \mathrm{Gyr})$.

HD 2057 and 2MASSI J0025036+475919-These L4 and F8 dwarfs appear to be common proper-motion companions separated by $3.6^{\prime}$ (7000-9000 AU). The Hipparcos proper motion for the F8,

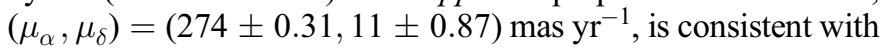
our measured value for the ultracool dwarf, $\left(\mu_{\alpha}, \mu_{\delta}\right)=(312 \pm$ $39,-9 \pm 44)$ mas $\mathrm{r}^{-1}$. While the projected physical separation is double that of the widest currently known multiple system with an ultracool dwarf component, Gl 584C at 3600 AU (Kirkpatrick et al. 2001a; Reid et al. 2001), it is not unreasonable based on the log-normal relation between maximum separation and the total mass of the system found by Reid et al. (2001).

Again, our spectrophotometric distance and the Hipparcos distance are discrepant. This time, however, we have already resolved the ultracool wide companion into an equal-luminosity binary (Reid et al. 2006a). Taking this binarity into account yields a spectrophotometric distance of $32 \pm 7 \mathrm{pc}$, within $1.5 \sigma$ of the Hipparcos distance of $42 \pm 2 \mathrm{pc}$. In addition, as mentioned above in $\S 5.1$, lithium absorption present in the spectrum of the ultracool dwarf implies an age less than $1 \mathrm{Gyr}$ and probably closer to 500 Myr. For HD 2057, Nordström et al. (2004) find an age of $\sim 1.1$ Gyr. However, with an upper limit of 3.6 Gyr and no lower limit given this age is uncertain.

$B D+131727$ and 2MASSI J0739438+130507.- These M8 and K5 dwarfs are a common proper-motion pair with a sepa- ration of $10.5^{\prime \prime}$ (380 AU). Their common proper motions were recognized while cross-referencing our sample with the Lépine and Shara Proper Motions (LSPM) catalog (Lépine \& Shara 2005). The Tycho-2 (Høg et al. 2000) proper motion for BD +13 1727, $\left(\mu_{\alpha}, \mu_{\delta}\right)=(-76.1 \pm 1.3,-156.5 \pm 1.2)$ mas $\mathrm{yr}^{-1}$, agrees well with the LSPM proper motion of the ultracool dwarf, $\left(\mu_{\alpha}, \mu_{\delta}\right)=$ $(-69 \pm 8,-145 \pm 8)$ mas $\mathrm{yr}^{-1}$. Our spectrophotometric distance for the M8 is $36.3 \pm 3.1 \mathrm{pc}$. No parallax or additional information on the primary could be found in the literature.

\subsection{Suspected Unresolved Ultracool Binaries}

Ultracool binaries with small separations, like wide systems, place constraints on star formation models (Burgasser et al. 2007). We have noticed two objects, originally presented in Paper V, that based on trigonometric parallax measurements appear to be significantly overluminous, suggesting that they are unresolved binaries. Both of these objects are being targeted for groundbased, high-resolution imaging.

These two objects were also misclassified by Reid et al. (1995, hereafter PMSU) as having earlier spectral types than revealed by our new spectra. The PMSU spectral types are based on the TiO5 index-spectral-type relation, which turns around at M7 (Cruz \& Reid 2002, their Fig. 3). Using the early-type branch of the calibration resulted in the assignment of a too-early spectral type. The spectral types estimated from our new spectra agree well with those predicted by the late-type TiO5-spectral-type relation derived by Cruz \& Reid.

LHS 1604 (2M 0351-00).-While PMSU assigned a spectral type of $\mathrm{M} 6(\mathrm{TiO} 5=0.18)$, our observations reveal a spectral type of M7.5 (Cruz et al. 2003). Using our spectral-type/ $M_{J}$ relation, we estimate $M_{J}=10.96 \pm 0.21$. However, the parallax of $68.1 \pm$ 1.8 mas (van Altena et al. 1995) combined with the 2MASS $J$ magnitude of $11.262 \pm 0.023$, implies $M_{J}=10.43 \pm 0.06$. This $\sim 0.6$ mag overluminosity suggests that the object might be an unresolved binary. We find that an M7.5/M9 pair would be consistent with the astrometric results. While this object is included in our total space density estimates, it is not included in our analysis of space density per magnitude because the parallax indicates a magnitude brighter than our brightest $M_{J}$ bin $\left(M_{J}=10.5\right)$.

LHS $3406(2 M 1843+40)$-For this object, PMSU estimated a spectral type of M5.5 ( TiO5 =0.24), while we find a spectral type of M8, indicating $M_{J}=11.16 \pm 0.18$ (Cruz et al. 2003). However, the parallax of $70.7 \pm 0.8$ (Monet et al. 1992) and the 2MASS $J$ magnitude of $11.299 \pm 0.028$ yield $M_{J}=10.55 \pm$ 0.04 . This overluminosity by $\sim 0.6$ mag could be explained if the object is an M8/M9 unresolved binary pair. In addition, this object was misclassified as a dwarf nova (U Gem variable; Downes et al. 1997) but has since been recognized as an $M$ dwarf by the cataclysmic-variable community (Liu et al. 1999). Unfortunately, we were unable to locate the original data that the dwarf nova classification was based on to investigate the possibility of a flare event.

\section{THE 20 pc 2MU2 SAMPLE AND THE LUMINOSITY FUNCTION}

As described below, the $2 \mathrm{MU} 2$ sample covers $36 \%$ of the celestial sphere. Within that area, we identify 99 objects in 91 systems with spectral types between M7 and L8 and estimated distances within 20 pc of the Sun; we dub these the 20 pc 2 MU2 sample. Over half of these systems were added to this sample through the observations described in this series of papers. Table 9 lists relevant data for all objects in the $20 \mathrm{pc} 2 \mathrm{MU} 2$ sample. In addition, we list systems with distance estimates within $1 \sigma$ of $20 \mathrm{pc}$ in Table 10. 

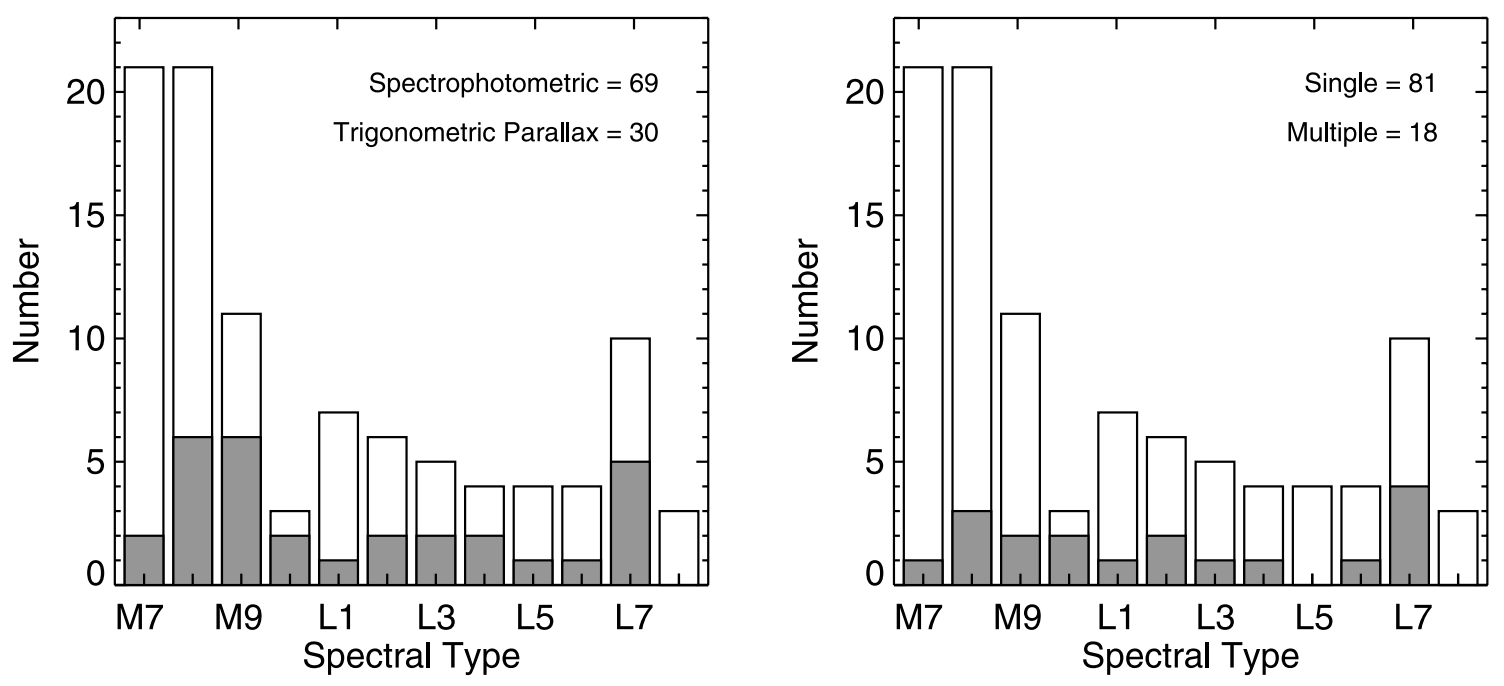

FIg. 10.- Spectral-type distribution of the $20 \mathrm{pc} 2 \mathrm{MU} 2$ sample. Left : Objects with distances based on trigonometric parallax measurements (shaded ) are distinguished from those with spectrophotometric distance estimates (not shaded). Right: Objects in multiple systems (shaded) are distinguished from single objects (not shaded).

In Figure 10, we show the spectral type distribution of the 20 pc sample with both the distance source and multiplicity properties distinguished. Trigonometric parallaxes are available for 30 systems; distances for the remaining sources are spectrophotometric. Among the 91 systems, 10 are multiple and contribute eight additional objects. The color-magnitude and color-color diagrams of the sample are shown in Figure 11. In Figure 12 we show the $\left(J-K_{S}\right)$ color distribution for different spectral types.

We use these data to build on the space-density analysis given in Paper V and derive an improved estimate of the $J$ - and $K$-band luminosity functions for ultracool dwarfs. We describe how we estimate our sky coverage in $\S 6.1$. Sample completeness is clearly an important factor in this analysis, and we address that issue in $\S 6$ 6.2. The Malmquist bias corrections appropriate to the sample are described in $\S 6.3$, binarity is discussed in $\S 6.4$, and the derived luminosity function is presented in $\S 6.5$.

\subsection{Areal Sky Coverage}

The total areal sky coverage of the 2MASS Second Incremental Data Release PSC is 19,641.6 deg ${ }^{2}$, imaged in 27,493 $6^{\circ} \times 8.5^{\prime}$ tiles. From this we excluded 3807 tiles with central Galactic latitudes within $10^{\circ}$ of the Galactic plane, reducing the coverage by $2878.1 \mathrm{deg}^{2}$. Parts of the remaining $16,763.5 \mathrm{deg}^{2}$ were excluded to eliminate star formation regions, the Magellanic Clouds, and other highly reddened or crowded regions (listed in Cruz et al. 2003, Tables 2 and 3). However, these regions were selected by Galactic coordinates, whereas the 2MASS imaging tiles are mapped in equatorial coordinates. In addition, many of these regions had only partial tile coverage in the 2MASS Second Incremental Data Release PSC.

In order to quantify the areal coverage excluded in these regions, we made use of the hist_nd algorithm written by J. D. Smith to create a histogram the coordinates of the point sources eliminated by the positional cuts onto a two-dimensional grid in a rectangular region aligned with the coordinate axes and of easily calculable area. The fraction of the nonzero elements in the resulting array was used as the estimate of the fraction of the area filled by the eliminated point sources and thus provided a measure of the area of the excluded region, taking into account the discontinuous sky coverage of the 2MASS Second Incremental Data Release PSC.
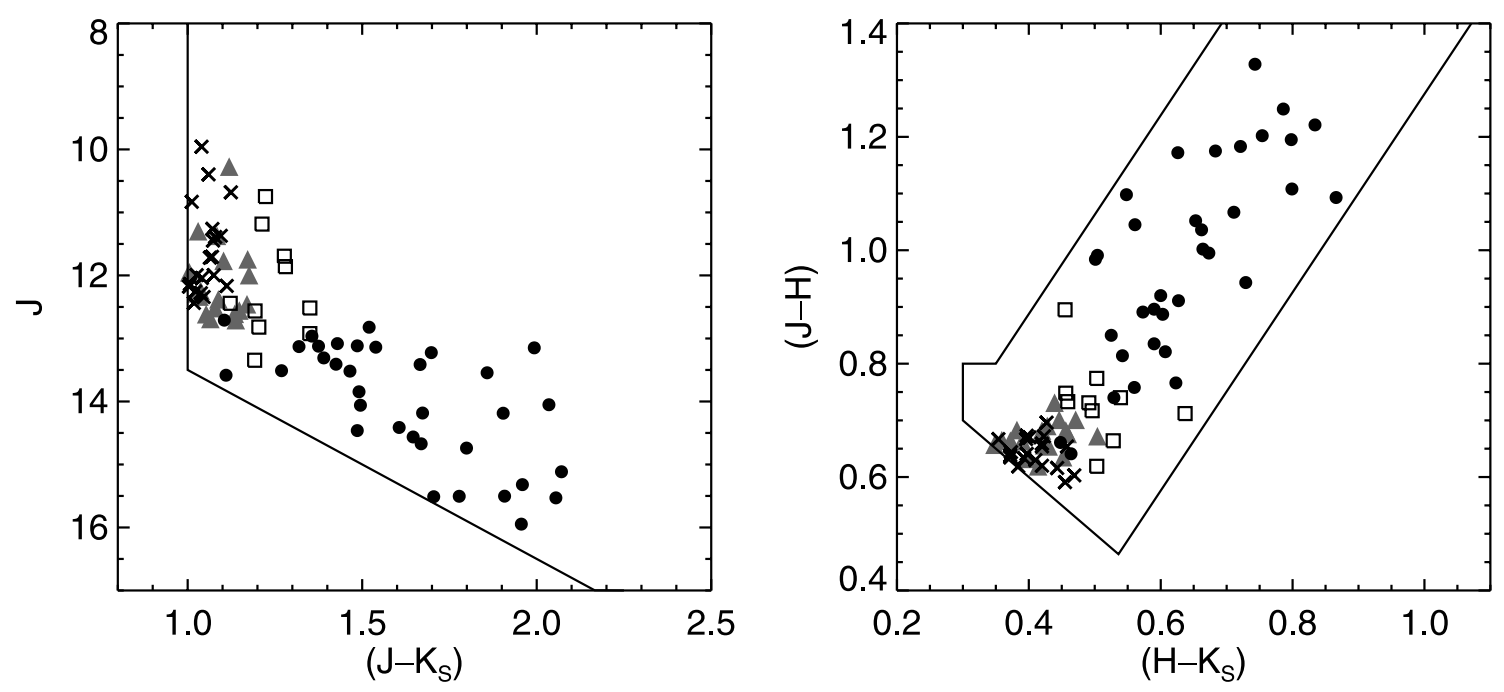

FIG. 11.-Color-magnitude and color-color selection criteria (solid lines) and distribution of the $20 \mathrm{pc} 2 \mathrm{MU} 2$ sample grouped by spectral type: M7 and M7.5 (crosses), M8 and M8.5 (triangles), M9 and M9.5 (squares), and L dwarfs (circles). The two blue L dwarfs near $\left(J-K_{S}\right)=1.1$ in the left panel and $\left(H-K_{S}\right)=0.45$ in the right panel are discussed in $\S 5.4$. 

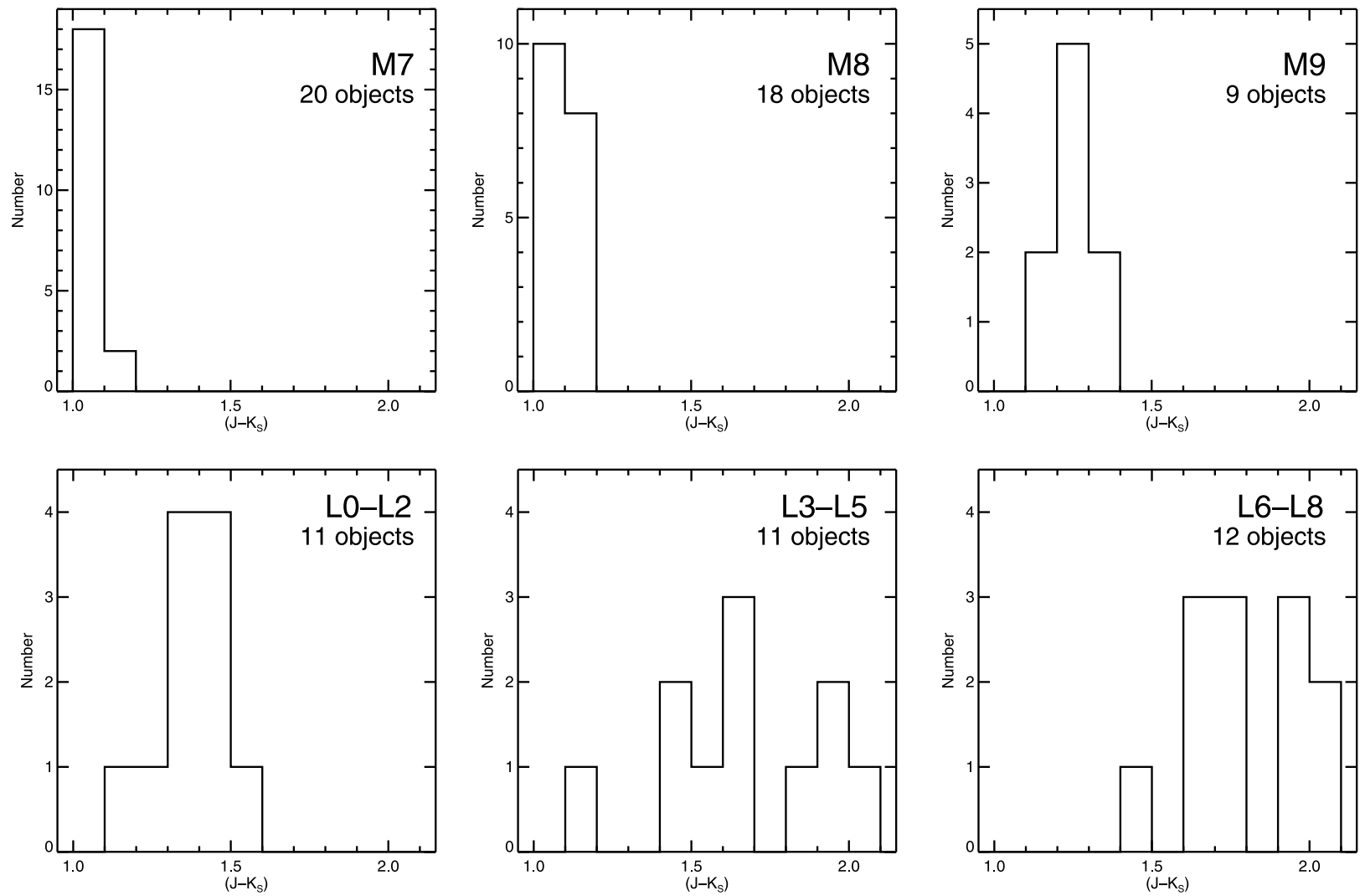

FIG. 12.- $\left(J-K_{S}\right)$ distribution for single dwarfs in the $20 \mathrm{pc} 2 \mathrm{MU} 2$ sample.

While the resulting measurement of the area is very sensitive to the histogram bin size chosen, the results are accurate to a few degrees, sufficient for our purposes. As many of the regions are aligned with the coordinate axes in one system but not in the other, the areas were measured in both Galactic and celestial systems to give an estimate of the uncertainties. The resulting measurement of the area removed due to positional cuts is $1940 \pm 10 \mathrm{deg}^{2}$.

Bright stars $(K<4)$ represent a further minor source of confusion, since their extended halos on the 2MASS scans rule out the possibility of detecting faint sources within as much as a few arcminutes. However, the total area obscured in this manner is less than $15 \mathrm{deg}^{2}$, producing negligible impact on our statistics. The final areal coverage of the 2MU2 sample is $14,823.5 \mathrm{deg}^{2}$, or $36 \%$ of the celestial sphere $\left(9 \%\right.$ smaller than the $16,350 \mathrm{deg}^{2}$ previously stated in Paper V).

\subsection{Completeness}

The 2MU2 sample has been carefully constructed, and extensive spectroscopic follow-up observations have been completed to yield a complete sample of M7-L8 type dwarfs to 20 pc over $36 \%$ of the sky. Below we discuss our observational, spectraltype, and resulting volume completeness.

\subsubsection{Observational Completeness}

As discussed in $\S 2$, we lack sufficient data for one object, 2MASSI J0028208+224905, that infrared spectroscopy identifies as a late-type L dwarf at $20-25$ pc. It is possible that optical spectroscopy might result in a spectral type of L7 or later, moving it within the $20 \mathrm{pc}$ limit. If this is the case, it would contribute to the 14.25 or $14.75 M_{J}$ bin of $\Phi\left(M_{J}\right)$. For this reason, and other more significant ones discussed in $\S 6.5$, our mea- surement of $\Phi\left(14<M_{J}<15\right)$ and $\Phi\left(12.5<M_{K_{S}}<13\right)$ are lower limits.

\subsubsection{Spectral-Type Incompleteness}

The 2MU2 sample is a color- and magnitude-defined sample. These selection criteria result in incompleteness at the extremes of the range of spectral type covered by our survey. For example, our sample is incomplete for M7 dwarfs due to the $\left(J-K_{S}\right)>$ 1.0 selection criterion; several M7 dwarfs are known with $\left(J-K_{S}\right)$ colors bluer than this limit, including VB 8, the M7 archetype.

We have quantified this incompleteness through analysis of a sample of ultracool dwarfs with $J<16.5$ and trigonometric parallax measurements from Dahn et al. (2002) or Vrba et al. (2004). We obtained photometry for these objects from the 2MASS Second Incremental Data Release PSC where available and otherwise used the data from the 2MASS All-Sky PSC. We refer to this sample as the "ultracool dwarf trigonometric" (UCDt) sample. Adjusting the measured $M_{J}$ to a distance of $20 \mathrm{pc}$ shows the systems as they would appear at the far edge of our volume limit. Figure 13 displays those data against our selection criteria, marking objects that are excluded. Blue M7 and M8 dwarfs, some L7 and L8 dwarfs, and unusual objects are likely to be excluded from the 2MU2 sample.

\subsubsection{Late-M Dwarfs}

Considering earlier types two M7 dwarfs in the UCDt sample, GRH 2208-2007 (M7.5) and VB 8 (M7), are excluded by both the $J /\left(J-K_{S}\right)$ and $(J-H) /\left(H-K_{S}\right)$ selection criteria. While all of the M8 dwarfs in the UCDt sample fall within our criteria, they also appear to be cut off by the $\left(J-K_{S}\right)=1.0$ limit (Fig. 11). To see this more clearly, in Figure 12 we show the $\left(J-K_{S}\right)$ 

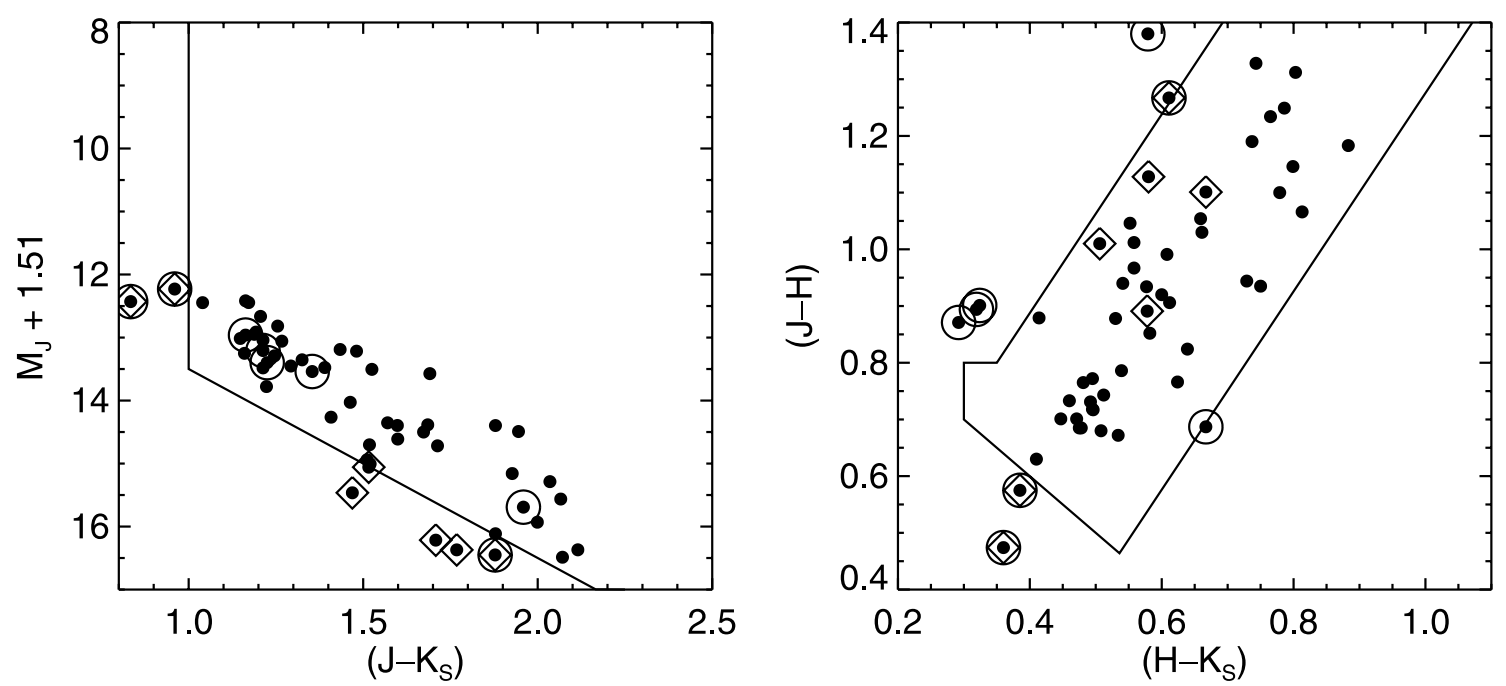

FIG. 13.-Color-magnitude and color-color diagram of M7-L8 dwarfs with parallax measurements and $J<16.5$ (UCDt sample, filled circles), and our selection criteria (solid lines). $M_{J}$ has been shifted by 1.51 to reflect our distance limit of $20 \mathrm{pc}$. Objects that are excluded by our selection criteria in $J /\left(J-K_{S}\right)($ diamonds) and $(J-H) /\left(H-K_{S}\right)$ (open circles) are marked in both panels and discussed in $\S$ 6.2.2.

distribution for M7, M8, and M9 dwarfs in the $20 \mathrm{pc} 2 \mathrm{MU} 2$ sample; while the M7 and M8 distributions appear truncated, the M9 distribution does not.

We cannot use the UCDt sample to estimate the resulting incompleteness, since it includes only three M7 and six M8 dwarfs. Instead, we have compiled data for M7 and M8 dwarfs listed in the literature that were not selected using $\left(J-K_{S}\right)$ color as a criterion. In Figure 14 we examine the $\left(J-K_{S}\right)$ distribution of these objects. Three of the $14 \mathrm{M} 7$ dwarfs have $\left(J-K_{S}\right)<1$, and we use a binomial distribution to estimate $78.6_{-14.3}^{+7} \%$ of M7 dwarfs are redder than $(J-K)=1$. Applying this correction to the observed number of $21 \mathrm{M} 7 \mathrm{dwarfs}$ in the $20 \mathrm{pc} 2 \mathrm{MU} 2$ sample yields a corrected number of $26.7 \mathrm{dwarfs}$, and the luminosity functions are changed accordingly. None of the $12 \mathrm{M} 8 \mathrm{dwarfs}$ found in the literature have $\left(J-K_{S}\right)<1$. While the $20 \mathrm{pc} 2 \mathrm{MU} 2$ sample is likely incomplete for M8 dwarfs the effect is small $(\sim 10 \%)$, and we do not correct the luminosity function for missing M8 dwarfs.

\subsubsection{Late-L Dwarfs}

At later types, the 2MU2 sample is incomplete since pressureinduced $\mathrm{H}_{2}$ absorption and perhaps dust settling (Knapp et al.

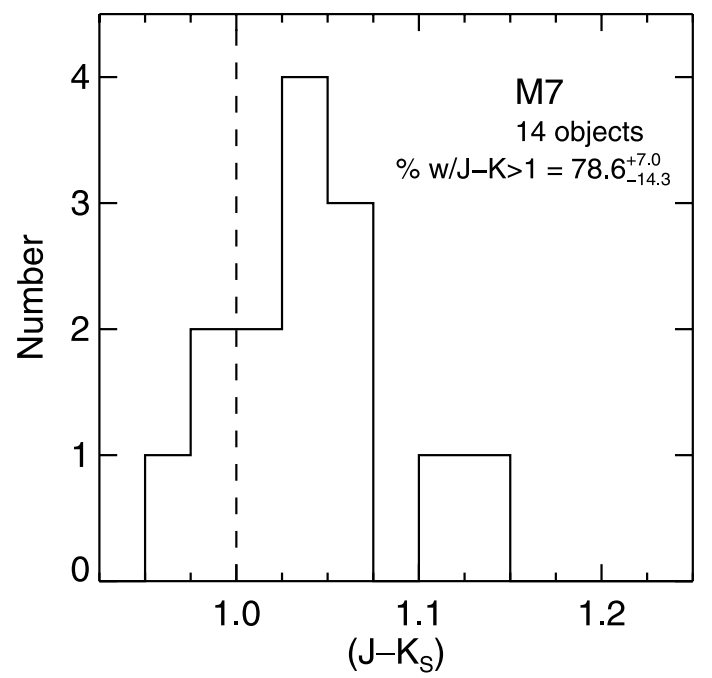

2004) leads to some L7 and L8 dwarfs having colors bluer than our selection criteria. The UCDt sample includes four L7 and six L8 dwarfs; of these, one L7.5 and four L8 dwarfs are excluded in the $\left[J,\left(J-K_{S}\right)\right]$ plane. While this incompleteness affects $14<$ $M_{J}<15$ and $12.5<M_{K_{S}}<13$, we have not computed explicit completeness corrections for those spectral types for two reasons. First, the incompleteness is by magnitude, and is therefore distance dependent; there is no incompleteness for late-type sources within $11 \mathrm{pc}$, for example. Second, and more significantly, early $\mathrm{T}$ dwarfs, which are not included in our survey, contribute to the space density of ultracool dwarfs in the same $M_{J}$ range as L7 and L8 dwarfs $\left(14<M_{J}<15\right)$. In the $K_{S}$ band, on the other hand, $\mathrm{T}$ dwarfs are fainter than L dwarfs and do not contribute to the space density in our $M_{K_{S}}$ range of interest (Vrba et al. 2004). Thus, we take our measured $\Phi\left(14<M_{J}<15\right)$ as a lower limit due to missing both $\mathrm{L}$ and $\mathrm{T}$ dwarfs, while $\Phi\left(12.5<M_{K_{S}}<13\right)$ is incomplete only due to missing late-L dwarfs.

\subsubsection{Unusual M9 and L0 Dwarfs}

One M9 dwarf and two L0 dwarfs in the UCDt sample have unusual $\left(H-K_{S}\right)$ colors $(\sim 0.35)$ : PC 0025+0447 (M9.5), SDSS

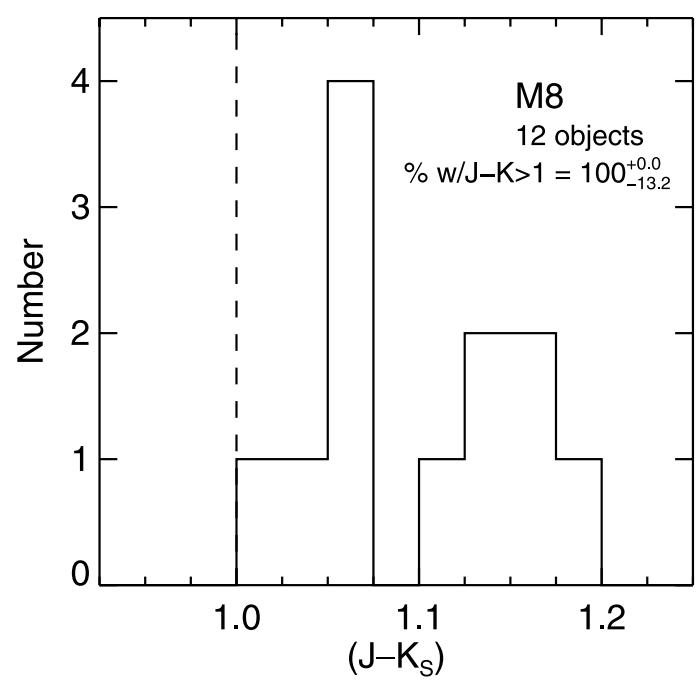

Fig. 14.- $\left(J-K_{S}\right)$ distribution for M7 and M8 dwarfs not discovered using $\left(J-K_{S}\right)$ color as a search criterion. The resulting fraction of M7 dwarfs with $\left(J-K_{S}\right)>1$ is applied as a correction to our derived $\Phi\left(M_{J}\right)$ and $\Phi\left(M_{K_{S}}\right)$. 
TABLE 3

M7-L8 Dwarfs Discovered outside 20 pc

\begin{tabular}{|c|c|c|c|c|c|c|c|c|c|c|c|}
\hline \multirow[b]{2}{*}{ 2MASSI Designation ${ }^{\mathrm{a}}$} & \multirow[b]{2}{*}{ 2MUCD No. } & \multirow[b]{2}{*}{ OtheR NAMES } & \multicolumn{3}{|c|}{ 2MASS } & \multirow[b]{2}{*}{ Obs. Date (UT) } & \multirow[b]{2}{*}{ Telescope } & \multirow{2}{*}{$\begin{array}{c}\text { Optical } \\
\text { Spectral Type }\end{array}$} & \multirow[b]{2}{*}{$M_{J}$} & \multirow{2}{*}{$\begin{array}{c}d \\
(\mathrm{pc})\end{array}$} & \multirow[b]{2}{*}{ OtHER REFS. } \\
\hline & & & $J$ & $J-H$ & $J-K_{S}$ & & & & & & \\
\hline $0000286-124515 \ldots \ldots \ldots$ & 10001 & 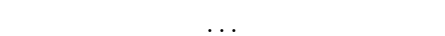 & 13.166 & 0.746 & 1.223 & $2002 \mathrm{Jul} 7$ & $\mathrm{KP} 2.1 \mathrm{~m}$ & M8.5 & $11.32 \pm 0.15$ & $23.4 \pm 1.7$ & \\
\hline $0003422-282241 \ldots \ldots \ldots$ & 10002 & HD $225118 \mathrm{~B} ?^{\mathrm{b}}$ & 13.063 & 0.699 & 1.108 & 2002 Sep 25 & KP 4 m & M7.5 & $10.96 \pm 0.21$ & $26.3 \pm 2.6$ & \\
\hline $0007078-245804 \ldots \ldots \ldots$ & 10003 & LEHPM 193 & 13.147 & 0.684 & 1.095 & 2002 Sep 26 & $\mathrm{KP} 4 \mathrm{~m}$ & M7 & $10.73 \pm 0.25$ & $30.4 \pm 3.5$ & 1,2 \\
\hline $0010001-203112 \ldots \ldots \ldots$. & 10006 & $\ldots$ & 14.153 & 0.761 & 1.285 & 2002 Sep 25 & $\mathrm{KP} 4 \mathrm{~m}$ & Lo & $11.73 \pm 0.13$ & $30.5 \pm 1.9$ & \\
\hline $0021162-664405 \ldots \ldots \ldots .$. & 13013 & $\ldots$ & 13.663 & 0.715 & 1.269 & 2004 Aug 9 & CT $4 \mathrm{~m}$ & M7:: & $10.73 \pm 1.01$ & $38.5 \pm 17.9$ & \\
\hline $0023475-323925 \ldots \ldots \ldots$. & 10017 & $\ldots$ & 12.646 & 0.641 & 1.002 & 2002 Sep 28 & $\mathrm{KP} 4 \mathrm{~m}$ & M7 & $10.73 \pm 0.25$ & $24.1 \pm 2.8$ & \\
\hline $0025036+475919 \ldots \ldots \ldots$. & 13016 & HD 2057B $?^{b}$ & 14.859 & 1.210 & 1.953 & 2004 Sep 22 & GN & L4: ${ }^{\mathrm{c}}$ & $13.09 \pm 0.22$ & $22.5 \pm 2.4$ & \\
\hline $0050244-153818 \ldots \ldots \ldots$. & 10045 & SIPS $0050-1538$ & 13.765 & 0.673 & 1.126 & 2002 Sep 26 & $\mathrm{KP} 4 \mathrm{~m}$ & L1: & $12.00 \pm 0.29$ & $22.5 \pm 3.0$ & 3 \\
\hline $0053540+500149 \ldots \ldots \ldots$. & 10049 & & 13.702 & 0.686 & 1.071 & 2002 Sep 25 & $\mathrm{KP} 4 \mathrm{~m}$ & M7.5 & $10.96 \pm 0.21$ & $35.3 \pm 3.4$ & \\
\hline $0055046-305200 \ldots \ldots \ldots$. & 10050 & $\ldots$ & 13.062 & 0.772 & 1.137 & 2002 Sep 26 & $\mathrm{KP} 4 \mathrm{~m}$ & M8: & $11.16 \pm 0.35$ & $24.1 \pm 3.9$ & \\
\hline $0103079+450929 \ldots \ldots \ldots$. & 10058 & $\ldots$ & 13.704 & 0.745 & 1.265 & 2002 Sep 25 & $\mathrm{KP} 4 \mathrm{~m}$ & M9 & $11.47 \pm 0.14$ & $28.0 \pm 1.9$ & \\
\hline $0107160-151757 \ldots \ldots \ldots$ & 10066 & $\ldots$ & 13.341 & 0.625 & 1.059 & 2002 Sep 26 & $\mathrm{KP} 4 \mathrm{~m}$ & M7 & $10.73 \pm 0.25$ & $33.2 \pm 3.9$ & \\
\hline $0112216-703123 \ldots \ldots \ldots . .$. & 13050 & $\ldots$ & 13.498 & 0.681 & 1.128 & 2003 Nov 9 & CT $1.5 \mathrm{~m}$ & M7: & $10.73 \pm 0.50$ & $35.7 \pm 8.3$ & \\
\hline $0121525-685518 \ldots \ldots \ldots$ & 13054 & $\ldots$ & 12.885 & 0.676 & 1.044 & 2003 Nov 8 & CT $1.5 \mathrm{~m}$ & M7 & $10.73 \pm 0.25$ & $26.9 \pm 3.1$ & \\
\hline $0131183+380155 \ldots \ldots \ldots$ & 13061 & $\ldots$ & 14.694 & 1.022 & 1.666 & $2004 \mathrm{Feb} 12$ & $\mathrm{KP} 4 \mathrm{~m}$ & L4: & $13.09 \pm 0.44$ & $20.9 \pm 4.3$ & \\
\hline $0230155+270406 \ldots \ldots \ldots$. & 10119 & $\ldots$ & 14.255 & 0.770 & 1.279 & $2002 \operatorname{Jan} 25,2002 \operatorname{Sep} 26$ & $\mathrm{KP} 4 \mathrm{~m}$ & L0: & $11.73 \pm 0.26$ & $32.0 \pm 3.9$ & \\
\hline $0310140-275645 \ldots \ldots \ldots$. & 10170 & $\ldots$ & 15.813 & 1.151 & 1.873 & $\begin{array}{l}2002 \text { Sep } 28 \\
2005 \text { Oct } 11\end{array}$ & $\begin{array}{l}\mathrm{KP} 4 \mathrm{~m} \\
\mathrm{GS}\end{array}$ & $\mathrm{L}^{\mathrm{c}}$ & $13.55 \pm 0.24$ & $28.3 \pm 3.2$ & \\
\hline $0320171-102612 \ldots \ldots \ldots .$. & 10177 & $\ldots$ & 13.865 & 0.747 & 1.149 & 2002 Sep 26 & $\mathrm{KP} 4 \mathrm{~m}$ & M8 & $11.16 \pm 0.18$ & $34.8 \pm 2.9$ & \\
\hline $0325013+225303 \ldots \ldots \ldots .$. & 13119 & $\ldots$ & 15.410 & 1.095 & 1.675 & 2004 Oct 19 & GN & L3 & $12.67 \pm 0.20$ & $35.3 \pm 3.3$ & \\
\hline $0342162-681732 \ldots \ldots \ldots$. & 10204 & $\ldots$ & 16.876 & 1.507 & 2.328 & 2004 Dec 11 & GS & L2: & $12.31 \pm 0.34$ & $81.9 \pm 13.8$ & \\
\hline $0355201+143929 \ldots \ldots \ldots$. & 10223 & $\ldots$ & 13.814 & 0.706 & 1.106 & 2002 Sep 28 & $\mathrm{KP} 4 \mathrm{~m}$ & M8 & $11.16 \pm 0.18$ & $34.0 \pm 2.9$ & \\
\hline $0407089-234829 \ldots \ldots \ldots$. & 10240 & $\ldots$ & 13.767 & 0.698 & 1.148 & 2002 Sep 26 & KP $4 \mathrm{~m}$ & M8: & $11.16 \pm 0.35$ & $33.3 \pm 5.4$ & \\
\hline $0417474-212919 \ldots \ldots \ldots$. & 10264 & $\ldots$ & 13.854 & 0.728 & 1.177 & 2002 Sep 26 & KP $4 \mathrm{~m}$ & M8 & $11.16 \pm 0.18$ & $34.7 \pm 2.9$ & \\
\hline $0421072-630602 \ldots \ldots \ldots$ & 10268 & $\ldots$ & 15.561 & 1.281 & 2.128 & 2004 Dec 12 & GS & $\mathrm{L} 4^{\mathrm{c}}$ & $13.09 \pm 0.22$ & $31.1 \pm 3.3$ & \\
\hline $0436501-180326 \ldots \ldots \ldots .$. & 10307 & $\ldots$ & 13.667 & 0.677 & 1.110 & 2002 Sep 25 & $\mathrm{KP} 4 \mathrm{~m}$ & M7 & $10.73 \pm 0.25$ & $38.6 \pm 4.5$ & \\
\hline $0518461-275645 \ldots \ldots \ldots$. & 10381 & $\ldots$ & 15.279 & 0.957 & 1.651 & 2002 Sep 28 & $\mathrm{KP} 4 \mathrm{~m}$ & L0: & $11.73 \pm 0.26$ & $51.3 \pm 6.3$ & \\
\hline 0518599-282837A........ & 10383 & $\ldots$ & 16.050 & 1.224 & 1.866 & & Keck I & L6.5 $\mathrm{pec}^{\mathrm{d}}$ & $13.9 \pm 0.3$ & $36 \pm 5$ & 4,5 \\
\hline 0518599-282837B....... & $\ldots$ & $\ldots$ & $\ldots$ & $\ldots \ldots$ & $\ldots$ & $\ldots$ & $\ldots$ & $\ldots$ & $14.0 \pm 0.3$ & $36 \pm 5$ & 4,6 \\
\hline $0534584-151143 \ldots \ldots \ldots .$. & 10396 & $\ldots$ & 13.151 & 0.636 & 1.154 & 2002 Sep 25 & KP $4 \mathrm{~m}$ & M9 & $11.47 \pm 0.14$ & $21.7 \pm 1.4$ & \\
\hline $0536199-192039 \ldots \ldots \ldots$. & 10397 & $\ldots$ & 15.791 & 1.107 & 1.919 & 2005 Jan 10 & GS & L1: & $12.00 \pm 0.29$ & $57.3 \pm 7.8$ & \\
\hline $0605019-234226 \ldots \ldots \ldots$. & 10499 & $\ldots$ & 14.505 & 0.778 & 1.344 & 2002 Sep 27 & $\mathrm{KP} 4 \mathrm{~m}$ & L0: & $11.73 \pm 0.26$ & $35.9 \pm 4.4$ & \\
\hline $0639559-741844 \ldots \ldots \ldots$ & 13147 & $\ldots$ & 15.794 & 1.105 & 1.778 & 2004 Dec 11, 2004 Dec 30 & GS & L5 & $13.55 \pm 0.24$ & $28.0 \pm 3.3$ & \\
\hline $0739438+130507 \ldots \ldots \ldots .$. & 10662 & $\mathrm{BD}+13$ 1727B?/LSPM J0739+1305 b & 13.957 & 0.613 & 1.214 & 2002 Sep 26 & $\mathrm{KP} 4 \mathrm{~m}$ & M8 & $11.16 \pm 0.18$ & $36.3 \pm 3.1$ & 7 \\
\hline $0821501+453201 \ldots \ldots \ldots$. & 10715 & $\ldots$ & 13.469 & 0.720 & 1.142 & $2004 \mathrm{Feb} 10$ & KP $4 \mathrm{~m}$ & M7.5 & $10.96 \pm 0.21$ & $31.7 \pm 3.1$ & \\
\hline $0953212-101420 \ldots \ldots \ldots$. & 10857 & $\ldots$ & 13.445 & 0.814 & 1.314 & 2003 Apr 22 & $\mathrm{CT} 4 \mathrm{~m}$ & L0 & $11.73 \pm 0.13$ & $22.0 \pm 1.4$ & \\
\hline $1011395+201903 \ldots \ldots \ldots \ldots$ & 10886 & $\ldots$ & 13.860 & 0.783 & 1.186 & 2003 Apr 22 & $\mathrm{CT} 4 \mathrm{~m}$ & M8 & $11.16 \pm 0.18$ & $34.7 \pm 2.9$ & \\
\hline $1028404-143843 \ldots \ldots \ldots$. & 10910 & & 13.091 & 0.643 & 1.064 & 2002 Jan 25, 2003 Apr 21 & CT $4 \mathrm{~m}$ & $\mathrm{M} 7^{\mathrm{e}}$ & $10.73 \pm 0.25$ & $29.6 \pm 3.5$ & 8 \\
\hline $1128255+783101 \ldots \ldots \ldots .$. & 10992 & LSPM J1128+7831 & 13.397 & 0.619 & 1.007 & 2002 Jul 8 & $\mathrm{KP} 2.1 \mathrm{~m}$ & M7: & $10.73 \pm 0.50$ & $34.1 \pm 7.9$ & 7,9 \\
\hline $1147048+142009$.......... & 11012 & LSPM J1147+1420 & 13.296 & 0.637 & 1.041 & $2002 \mathrm{Jul} 7$ & $\mathrm{KP} 2.1 \mathrm{~m}$ & M7 & $10.73 \pm 0.25$ & $32.5 \pm 3.8$ & 7 \\
\hline $1216216+445634 \ldots \ldots \ldots .$. & 11053 & $\ldots$ & 16.763 & 1.293 & 2.113 & 2004 Nov 11 & GN & L5 & $13.55 \pm 0.24$ & $43.8 \pm 6.4$ & \\
\hline $1221506-084319 \ldots \ldots \ldots$ & 11062 & $\ldots$ & 13.534 & 0.613 & 1.014 & $2002 \mathrm{Jul} 7$ & $\mathrm{KP} 2.1 \mathrm{~m}$ & M8 & $11.16 \pm 0.18$ & $29.9 \pm 2.5$ & \\
\hline $1231214+495923 \ldots \ldots \ldots .$. & 11076 & $\ldots$ & 14.639 & 0.949 & 1.488 & $2004 \mathrm{Feb} 11$ & $\mathrm{KP} 4 \mathrm{~m}$ & $\mathrm{~L} 2$ & $12.31 \pm 0.17$ & $29.2 \pm 2.3$ & \\
\hline
\end{tabular}


TABLE 3-Continued

\begin{tabular}{|c|c|c|c|c|c|c|c|c|c|c|c|}
\hline \multirow[b]{2}{*}{ 2MASSI Designation ${ }^{\mathrm{a}}$} & \multirow[b]{2}{*}{ 2MUCD No. } & \multirow[b]{2}{*}{ OtHER NAMES } & \multicolumn{3}{|c|}{ 2MASS } & \multirow[b]{2}{*}{ OBs. Date (UT) } & \multirow[b]{2}{*}{ Telescope } & \multirow{2}{*}{$\begin{array}{c}\text { Optical } \\
\text { Spectral Type }\end{array}$} & \multirow[b]{2}{*}{$M_{J}$} & \multirow{2}{*}{$\begin{array}{c}d \\
(\mathrm{pc})\end{array}$} & \multirow[b]{2}{*}{ Other ReFs. } \\
\hline & & & $J$ & $J-H$ & $J-K_{S}$ & & & & & & \\
\hline $1303239+360249$............ & 11117 & $\ldots$ & 13.646 & 0.779 & 1.136 & 2002 Apr 10 & APO & M8 & $11.16 \pm 0.18$ & $31.5 \pm 2.6$ & \\
\hline $1312070+393744 \ldots \ldots \ldots .$. & 11130 & $\ldots$ & 14.146 & 0.741 & 1.250 & 2002 Apr 10 & APO & L0: & $11.73 \pm 0.26$ & $30.4 \pm 3.7$ & \\
\hline $1323521+301433 \ldots \ldots \ldots$ & 11139 & $\ldots$ & 13.681 & 0.608 & 1.103 & 2002 Apr 10 & APO & M8.5 & $11.32 \pm 0.15$ & $29.7 \pm 2.2$ & \\
\hline $1332234+154219 \ldots \ldots \ldots$ & 11152 & $\ldots$ & 13.509 & 0.671 & 1.054 & 2002 Jul 7 & $\mathrm{KP} 2.1 \mathrm{~m}$ & M7 & $10.73 \pm 0.25$ & $35.9 \pm 4.2$ & \\
\hline $1336406+374323 \ldots \ldots \ldots$ & 11156 & $\ldots$ & 14.397 & 0.778 & 1.313 & $2002 \mathrm{Jul} 10,2002 \mathrm{Jul} 11$ & APO & $\mathrm{L} 1^{\mathrm{e}}$ & $12.00 \pm 0.14$ & $30.2 \pm 2.0$ & \\
\hline $1337311+493836 \ldots \ldots \ldots$ & 11157 & $\ldots$ & 13.739 & 0.668 & 1.165 & $\begin{array}{l}2002 \text { May } 14 \\
2003 \text { Jul } 10\end{array}$ & $\begin{array}{l}\mathrm{APO} \\
\mathrm{KP} 4 \mathrm{~m}\end{array}$ & L0 & $11.73 \pm 0.13$ & $25.2 \pm 1.6$ & \\
\hline $1357096+554449 \ldots \ldots \ldots$ & 11179 & $\ldots$ & 14.152 & 0.823 & 1.304 & 2002 Apr 10 & $\mathrm{APO}$ & M9 & $11.47 \pm 0.14$ & $34.5 \pm 2.3$ & \\
\hline $1357149-143852 \ldots \ldots \ldots$ & 11180 & DENIS-P J1357-1438 & 12.852 & 0.654 & 1.107 & 2003 Mar 13 & $\mathrm{KP} 2.1 \mathrm{~m}$ & M7 & $10.73 \pm 0.25$ & $26.5 \pm 3.1$ & 10 \\
\hline $1404449+463429 \ldots \ldots \ldots$ & 11190 & $\ldots$ & 14.352 & 0.814 & 1.301 & $2002 \mathrm{Jul} 11$ & APO & L0: & $11.73 \pm 0.26$ & $33.4 \pm 4.1$ & \\
\hline $1405040+291831 \ldots \ldots \ldots$. & 11191 & $\ldots$ & 13.451 & 0.684 & 1.009 & $2002 \mathrm{Jul} 7$ & $\mathrm{KP} 2.1 \mathrm{~m}$ & M7 & $10.73 \pm 0.25$ & $34.9 \pm 4.1$ & \\
\hline $1412227+235410 \ldots \ldots \ldots \ldots$ & 11196 & $\ldots$ & 13.771 & 0.706 & 1.099 & $2002 \mathrm{Jul} 7$ & $\mathrm{KP} 2.1 \mathrm{~m}$ & M9 & $11.47 \pm 0.14$ & $28.9 \pm 1.9$ & \\
\hline $1415202+463659 \ldots \ldots \ldots$ & 11199 & $\ldots$ & 14.185 & 0.776 & 1.241 & 2002 May 30 & APO & M9 & $11.47 \pm 0.14$ & $35.0 \pm 2.3$ & \\
\hline $1434582-233557 \ldots \ldots \ldots$ & 11219 & CE 455 & 12.900 & 0.614 & 1.025 & 2002 Jul 6 & $\mathrm{KP} 2.1 \mathrm{~m}$ & M7 & $10.73 \pm 0.25$ & $27.1 \pm 3.2$ & 11 \\
\hline $1440303+123334 \ldots \ldots \ldots$. & 11231 & $\ldots$ & 14.427 & 0.848 & 1.316 & 2002 Jul 11 & APO & M9 & $11.47 \pm 0.14$ & $39.1 \pm 2.6$ & \\
\hline $1441045+271932 \ldots \ldots \ldots$. & 11233 & $\ldots$ & 13.022 & 0.609 & 1.033 & 2002 Jul 5 & $\mathrm{KP} 2.1 \mathrm{~m}$ & M7 & $10.73 \pm 0.25$ & $28.7 \pm 3.4$ & \\
\hline $1453230+154308 \ldots \ldots \ldots .$. & 11259 & $\ldots$ & 13.222 & 0.636 & 1.019 & 2002 Jul 5 & $\mathrm{KP} 2.1 \mathrm{~m}$ & M7.5 & $10.96 \pm 0.21$ & $28.3 \pm 2.8$ & \\
\hline $1453484+373316 \ldots \ldots \ldots$ & 11260 & $\ldots$ & 13.170 & 0.638 & 1.034 & 2002 Jul 5 & $\mathrm{KP} 2.1 \mathrm{~m}$ & M7 & $10.73 \pm 0.25$ & $30.7 \pm 3.6$ & \\
\hline $1510295+361948 \ldots \ldots \ldots$. & 11300 & $\ldots$ & 13.963 & 0.736 & 1.174 & $2002 \mathrm{Jul} 10$ & APO & M9 & $11.47 \pm 0.14$ & $31.6 \pm 2.1$ & \\
\hline $1536191+330514 \ldots \ldots \ldots$. & 11351 & $\ldots$ & 13.663 & 0.676 & 1.056 & $2002 \mathrm{Jul} 5$ & $\mathrm{KP} 2.1 \mathrm{~m}$ & M7 & $10.73 \pm 0.25$ & $38.5 \pm 4.5$ & \\
\hline \multirow[t]{2}{*}{$1550084+145517 \ldots \ldots \ldots}$. & 11452 & $\ldots$ & 14.746 & 0.934 & 1.520 & 2002 Jul 11 & $\mathrm{APO}$ & L2: & $12.31 \pm 0.34$ & $30.7 \pm 4.8$ & \\
\hline & & & 14.746 & 0.934 & 1.520 & 2003 Apr 21 & CT $4 \mathrm{~m}$ & L2: & $12.31 \pm 0.34$ & $30.7 \pm 4.8$ & \\
\hline $1556502+520656 \ldots \ldots \ldots$ & 11474 & $\ldots$ & 13.883 & 0.707 & 1.134 & 2002 Jul 10 & APO & M7 & $10.73 \pm 0.25$ & $42.6 \pm 5.0$ & \\
\hline $1557327+175238 \ldots \ldots \ldots$. & 11478 & $\ldots$ & 13.536 & 0.718 & 1.087 & $2002 \mathrm{Jul} 4$ & $\mathrm{KP} 2.1 \mathrm{~m}$ & M7.5 & $10.96 \pm 0.21$ & $32.7 \pm 3.2$ & \\
\hline $1607152+312525 \ldots \ldots \ldots$ & 11511 & $\ldots$ & 12.745 & 0.696 & 1.018 & $2002 \mathrm{Jul} 4$ & $\mathrm{KP} 2.1 \mathrm{~m}$ & M6 & $10.12 \pm 0.37$ & $33.5 \pm 5.8$ & \\
\hline $1608246+195747 \ldots \ldots \ldots \ldots$ & 11515 & $\ldots$ & 13.521 & 0.712 & 1.142 & 2002 May 30 & APO & M9 & $11.47 \pm 0.14$ & $25.8 \pm 1.7$ & \\
\hline $1612413+173028 \ldots \ldots \ldots$. & 11528 & $\ldots$ & 13.678 & 0.591 & 1.096 & 2002 Jul 7 & $\mathrm{KP} 2.1 \mathrm{~m}$ & M7 & $10.73 \pm 0.25$ & $38.8 \pm 4.5$ & \\
\hline $1613455+170827 \ldots \ldots \ldots$. & 11531 & $\ldots$ & 13.438 & 0.757 & 1.290 & 2002 May 30 & APO & M9.5 & $11.60 \pm 0.13$ & $23.3 \pm 1.5$ & \\
\hline $1617003+131349 \ldots \ldots \ldots$ & 11549 & $\ldots$ & 13.328 & 0.569 & 1.010 & $2002 \mathrm{Jul} 4$ & $\mathrm{KP} 2.1 \mathrm{~m}$ & M7 & $10.73 \pm 0.25$ & $33.0 \pm 3.8$ & \\
\hline $1645220+300407 \ldots \ldots \ldots .$. & 11658 & $\ldots$ & 15.217 & 1.019 & 1.669 & 2004 Sep 19 & GN & L3 & $12.67 \pm 0.20$ & $32.3 \pm 3.0$ & \\
\hline $1711135+232633 \ldots \ldots \ldots$. & 11684 & $\ldots$ & 14.512 & 0.865 & 1.460 & 2002 Sep 27 & $\mathrm{KP} 4 \mathrm{~m}$ & L0: & $11.73 \pm 0.26$ & $36.0 \pm 4.4$ & \\
\hline $1717045+150953 \ldots \ldots \ldots$ & 11690 & $\ldots$ & 13.612 & 0.659 & 1.112 & 2002 Sep 25 & $\mathrm{KP} 4 \mathrm{~m}$ & M7 & $10.73 \pm 0.25$ & $37.6 \pm 4.4$ & \\
\hline $1923381-330841 \ldots \ldots \ldots$ & 11842 & $\ldots$ & 13.278 & 0.609 & 1.039 & $2002 \mathrm{Jul} 4$ & $\mathrm{KP} 2.1 \mathrm{~m}$ & M7 & $10.73 \pm 0.25$ & $32.3 \pm 3.8$ & \\
\hline $2025196-255048 \ldots \ldots \ldots$ & 12003 & $\ldots$ & 14.075 & 0.693 & 1.210 & 2002 Jul 11 & APO & M8 & $11.16 \pm 0.18$ & $38.4 \pm 3.2$ & \\
\hline \multirow[t]{2}{*}{$2026158-294312 \ldots \ldots \ldots$} & 12009 & $\ldots$ & 14.800 & 0.867 & 1.441 & 2002 Sep 27 & $\mathrm{KP} 4 \mathrm{~m}$ & L1: & $12.00 \pm 0.29$ & $36.3 \pm 4.8$ & \\
\hline & & & & & & 2003 Apr 21 & CT $4 \mathrm{~m}$ & & & & \\
\hline $2035203-311008 \ldots \ldots \ldots .$. & 12026 & $\ldots$ & 13.195 & 0.608 & 1.060 & $2002 \mathrm{Jul} 4$ & $\mathrm{KP} 2.1 \mathrm{~m}$ & M7 & $10.73 \pm 0.25$ & $31.1 \pm 3.6$ & \\
\hline $2041428-350644 \ldots \ldots \ldots$ & 12037 & $\ldots$ & 14.863 & 0.895 & 1.480 & 2002 Sep 26 & $\mathrm{KP} 4 \mathrm{~m}$ & L2: & $12.31 \pm 0.34$ & $32.4 \pm 5.0$ & \\
\hline $2047247+142152 \ldots \ldots \ldots \ldots$ & 12044 & $\ldots$ & 13.006 & 0.713 & 1.158 & $2002 \mathrm{Jul} 4$ & $\mathrm{KP} 2.1 \mathrm{~m}$ & M7.5 & $10.96 \pm 0.21$ & $25.6 \pm 2.5$ & \\
\hline $2123311-234518 \ldots \ldots \ldots .$. & 12077 & $\ldots$ & 13.584 & 0.707 & 1.044 & $2002 \mathrm{Jul} 4$ & $\mathrm{KP} 2.1 \mathrm{~m}$ & M7.5 & $10.96 \pm 0.21$ & $33.4 \pm 3.3$ & \\
\hline $2132114+134158 \ldots \ldots \ldots .$. & 13177 & $\ldots$ & 15.785 & 1.201 & 1.951 & 2004 Sep 20 & GN & L6 & $14.02 \pm 0.30$ & $22.5 \pm 3.2$ & \\
\hline $2151254-244100 \ldots \ldots \ldots$ & 12091 & $\ldots$ & 15.775 & 1.197 & 2.102 & 2004 Nov 11 & GS & L3 & $12.67 \pm 0.20$ & $41.7 \pm 4.1$ & \\
\hline $2158045-155009 \ldots \ldots \ldots$ & 12101 & $\ldots$ & 14.949 & 1.033 & 1.801 & 2002 Sep 26 & $\mathrm{KP} 4 \mathrm{~m}$ & L4: & $13.09 \pm 0.44$ & $23.5 \pm 4.8$ & 12 \\
\hline $2242531+254257 \ldots \ldots \ldots$. & 13206 & $\ldots$ & 14.795 & 1.041 & 1.773 & 2004 Nov 6 & GN & L3 & $12.67 \pm 0.20$ & $26.6 \pm 2.5$ & \\
\hline
\end{tabular}


TABLE 3-Continued

\begin{tabular}{|c|c|c|c|c|c|c|c|c|c|c|c|}
\hline \multirow[b]{2}{*}{ 2MASSI Designation ${ }^{\mathrm{a}}$} & \multirow[b]{2}{*}{ 2MUCD No. } & \multirow[b]{2}{*}{ OtHeR NAMES } & \multicolumn{3}{|c|}{ 2MASS } & \multirow[b]{2}{*}{ Obs. Date (UT) } & \multirow[b]{2}{*}{ Telescope } & \multirow{2}{*}{$\begin{array}{c}\text { Optical } \\
\text { Spectral Type }\end{array}$} & \multirow[b]{2}{*}{$M_{J}$} & \multirow{2}{*}{$\begin{array}{c}d \\
(\mathrm{pc})\end{array}$} & \multirow[b]{2}{*}{ OTHER REFS. } \\
\hline & & & $J$ & $J-H$ & $J-K_{S}$ & & & & & & \\
\hline $2249091+320549 \ldots \ldots \ldots \ldots \ldots \ldots$ & 13209 & $\ldots$ & 15.489 & 1.133 & 1.896 & 2004 Sep 21 & GN & L5 & $13.55 \pm 0.24$ & $24.4 \pm 2.8$ & \\
\hline $2308099-313122 \ldots \ldots \ldots \ldots \ldots \ldots . .$. & 12172 & $\ldots$ & 13.666 & 0.709 & 1.073 & 2002 Jul 11 & APO & M7 & $10.73 \pm 0.25$ & $38.6 \pm 4.5$ & \\
\hline $2310185-175909$.................... & 12177 & SSSPM $2310-1759$ & 14.397 & 0.819 & 1.388 & 2002 Sep 25 & $\mathrm{KP} 4 \mathrm{~m}$ & L0: & $11.73 \pm 0.26$ & $34.1 \pm 4.1$ & 13 \\
\hline $2323134-024435 \ldots \ldots \ldots \ldots \ldots$ & 12183 & $\ldots$ & 13.582 & 0.680 & 1.122 & $2002 \mathrm{Jul} 5$ & $\mathrm{KP} 2.1 \mathrm{~m}$ & M8.5 & $11.32 \pm 0.15$ & $28.3 \pm 2.1$ & \\
\hline $2329479-160755 \ldots \ldots \ldots \ldots \ldots \ldots . .$. & 12189 & $\ldots$ & 13.334 & 0.609 & 1.088 & 2002 Jul 5 & $\mathrm{KP} 2.1 \mathrm{~m}$ & M9 & $11.47 \pm 0.14$ & $23.6 \pm 1.5$ & \\
\hline $2330225-034718$.................... & 12190 & $\ldots$ & 14.526 & 0.797 & 1.419 & 2002 Sep 26 & $\mathrm{KP} 4 \mathrm{~m}$ & L1: & $12.00 \pm 0.29$ & $32.0 \pm 4.2$ & \\
\hline $2337166-093324$..................... & 12201 & $\cdots$ & 13.411 & 0.698 & 1.129 & $2002 \mathrm{Jul} 5$ & $\mathrm{KP} 2.1 \mathrm{~m}$ & M7.5 & $10.96 \pm 0.21$ & $30.9 \pm 3.0$ & \\
\hline $2341286-113335 \ldots \ldots \ldots \ldots \ldots \ldots \ldots$ & 12208 & $\cdots$ & 13.609 & 0.677 & 1.068 & 2002 Jul 6 & $\mathrm{KP} 2.1 \mathrm{~m}$ & M8 & $11.16 \pm 0.18$ & $31.0 \pm 2.6$ & \\
\hline $2344062-073328$.................. & 12212 & $\ldots$ & 14.850 & 0.986 & 1.621 & 2002 Sep 26 & $\mathrm{KP} 4 \mathrm{~m}$ & L4.5 & $13.32 \pm 0.23$ & $20.2 \pm 2.2$ & \\
\hline $2346547-315353$.................... & 12215 & SIPS 2346-3153 & 13.296 & 0.640 & 1.091 & 2002 Jul 5 & $\mathrm{KP} 2.1 \mathrm{~m}$ & M8 & $11.16 \pm 0.18$ & $26.8 \pm 2.2$ & 3 \\
\hline $2352050-110043 \ldots \ldots \ldots \ldots \ldots \ldots$ & 12221 & $\ldots$ & 12.868 & 0.693 & 1.146 & 2002 Jul 6 & $\mathrm{KP} 2.1 \mathrm{~m}$ & M7 & $10.73 \pm 0.25$ & $26.7 \pm 3.1$ & \\
\hline $2353594-083331$.................... & 12223 & DENIS-P J2353-0833 & 13.062 & 0.705 & 1.117 & 2002 Sep 25 & $\mathrm{KP} 4 \mathrm{~m}$ & M8.5 & $11.32 \pm 0.15$ & $22.3 \pm 1.6$ & 10 \\
\hline
\end{tabular}

Noте.-Table 3 is also available in machine-readable form in the electronic edition of the Astronomical Journal.

a The 2MASS designation is 2MASSI Jhhmmss[.]s \pm ddmmss.

Ultracool common proper-motion companion to a star as discussed in $\S 5.5$.

${ }^{c}$ Spectrum shows lithium absorption line, as shown in Fig. 2 and discussed in $\S 5.1$.

Probable L/T binary (Cruz et al. 2004; Burgasser et al. 2006b). Combined-light optical spectral type from J. Kirkpatrick et al. (2007a, in preparation).

Variable and/or flaring object that will be discussed in detail in Schmidt et al. (2006).

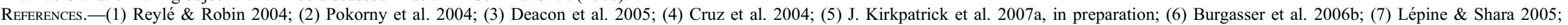

(8) Schmidt et al. 2006; (9) Lépine 2005; (10) Phan-Bao et al. 2001; (11) Ruiz et al. 2001; (12) J. Kirkpatrick et al. 2007b, in preparation; (13) Lodieu et al. 2002. 
TABLE 4

Early-to-Mid-Type M Dwarfs Discovered outside $20 \mathrm{pc}$

\begin{tabular}{|c|c|c|c|c|c|c|c|c|c|c|}
\hline \multirow[b]{2}{*}{ 2MASSI Designation ${ }^{\mathrm{a}}$} & \multirow[b]{2}{*}{ Other NAMES } & \multicolumn{3}{|c|}{ 2MASS } & \multirow[b]{2}{*}{ Obs. Date (UT) } & \multirow[b]{2}{*}{ Telescope } & \multirow{2}{*}{$\begin{array}{c}\text { Optical } \\
\text { SPECTRAL Type }\end{array}$} & \multirow[b]{2}{*}{$M_{J}$} & \multirow{2}{*}{$\begin{array}{c}d \\
(\mathrm{pc})\end{array}$} & \multirow[b]{2}{*}{ OtheR REFS. } \\
\hline & & $J$ & $J-H$ & $J-K_{S}$ & & & & & & \\
\hline $0017185-040606 \ldots \ldots$ & $\ldots$ & 12.485 & 0.642 & 1.025 & $2002 \mathrm{Jul} 7$ & $\mathrm{KP} 2.1 \mathrm{~m}$ & M6.5 & $10.5 \pm 0.3$ & $25 \pm 4$ & \\
\hline $0107590-200423 \ldots \ldots$. & $\ldots$ & 12.817 & 0.614 & 1.024 & 2002 Sep 27 & KP 4 m & M6 & $10.1 \pm 0.4$ & $35 \pm 6$ & \\
\hline $0355403-112310 \ldots \ldots \ldots$ & $\ldots$ & 12.947 & 0.645 & 1.010 & 2002 Sep 27 & $\mathrm{KP} 4 \mathrm{~m}$ & M6 & $10.1 \pm 0.4$ & $37 \pm 6$ & \\
\hline $0411063+124748 \ldots \ldots .$. & $\ldots$ & 12.777 & 0.652 & 1.015 & 2002 Sep 28 & $\mathrm{KP} 4 \mathrm{~m}$ & M6 & $10.1 \pm 0.4$ & $34 \pm 6$ & \\
\hline $0510239-280053 \ldots \ldots$ & $\ldots$ & 12.964 & 0.646 & 1.010 & 2002 Sep 28 & $\mathrm{KP} 4 \mathrm{~m}$ & M4 & $8.5 \pm 0.7$ & $60-100$ & \\
\hline $0544167-204909 \ldots \ldots$ & $\ldots$ & 14.394 & 1.002 & 1.515 & 2002 Sep 25 & $\mathrm{KP} 4 \mathrm{~m}$ & M5 & $8.5 \pm 0.7$ & $110-180$ & \\
\hline $0818332-003628 \ldots \ldots$. & $\ldots$ & 16.639 & 1.302 & 2.110 & 2004 Dec 12 & GS & M6 & $10.1 \pm 0.4$ & $202 \pm 38$ & \\
\hline $0941115+425403 \ldots \ldots \ldots$ & & 16.753 & 1.288 & 2.101 & 2004 Nov 7 & GN & M5 & $9.3 \pm 0.4$ & $312 \pm 59$ & \\
\hline $0959560+200234 \ldots \ldots \ldots$ & LHS 2215 & 12.244 & 0.629 & 1.048 & 2001 Nov 3 & $\mathrm{KP} 2.1 \mathrm{~m}$ & $\mathrm{M} 6.5^{\mathrm{b}}$ & $10.46 \pm 0.31$ & $22.8 \pm 3.2$ & $1,2,3,4,5$ \\
\hline $1144050+604348 \ldots \ldots \ldots$ & & 12.290 & 0.598 & 1.015 & $2002 \mathrm{Jul} 8$ & $\mathrm{KP} 2.1 \mathrm{~m}$ & M6 & $10.1 \pm 0.4$ & $27 \pm 5$ & \\
\hline $1222143+565559 \ldots \ldots \ldots$ & LSPM J1222+5655 & 13.662 & 0.697 & 1.099 & 2002 Jul 8 & $\mathrm{KP} 2.1 \mathrm{~m}$ & M6 & $10.1 \pm 0.4$ & $51 \pm 9$ & 6 \\
\hline $1239285+134142 \ldots \ldots .$. & LSPM J1239+1341 & 13.595 & 0.705 & 1.042 & $2002 \mathrm{Jul} 7$ & $\mathrm{KP} 2.1 \mathrm{~m}$ & M6 & $10.1 \pm 0.4$ & $50 \pm 9$ & 6 \\
\hline $1242271+445140 \ldots \ldots \ldots$ & LSPM J1242+4451 & 13.592 & 0.615 & 1.088 & $2002 \mathrm{Jul} 8$ & $\mathrm{KP} 2.1 \mathrm{~m}$ & M5 & $8.5 \pm 0.7$ & $75-130$ & 6 \\
\hline $1312393+183559 \ldots \ldots \ldots$ & LSPM J1312+1835 & 13.238 & 0.654 & 1.115 & 2002 Apr 10 & APO & M5 & $9.6 \pm 0.3$ & $54 \pm 7$ & 6 \\
\hline $1411392+602447 \ldots \ldots \ldots$ & & 13.461 & 0.684 & 1.016 & 2002 Jul 7 & $\mathrm{KP} 2.1 \mathrm{~m}$ & M6 & $10.1 \pm 0.4$ & $47 \pm 8$ & \\
\hline $1414153-141822 \ldots \ldots$. & & 13.499 & 0.559 & 1.014 & $2002 \mathrm{Jul} 6$ & $\mathrm{KP} 2.1 \mathrm{~m}$ & M6 & $10.1 \pm 0.4$ & $47 \pm 8$ & \\
\hline $1436418-153048 \ldots \ldots$. & $\ldots$ & 13.128 & 0.626 & 1.010 & $2002 \mathrm{Jul} 6$ & $\mathrm{KP} 2.1 \mathrm{~m}$ & M5 & $8.5 \pm 0.7$ & $65-115$ & \\
\hline $1450366+472357 \ldots \ldots \ldots$ & LSPM J1450+4723 & 13.374 & 0.663 & 1.012 & $2002 \mathrm{Jul} 5$ & $\mathrm{KP} 2.1 \mathrm{~m}$ & M6 & $10.1 \pm 0.4$ & $45 \pm 8$ & 6 \\
\hline $1529456+821532 \ldots \ldots \ldots$ & $\ldots$ & 13.437 & 0.610 & 1.032 & $2002 \mathrm{Jul} 5$ & $\mathrm{KP} 2.1 \mathrm{~m}$ & M5 & $6.6 \pm 0.5$ & $244 \pm 53$ & \\
\hline $1628170+133420 \ldots \ldots .$. & LSPM J1628+1334 & 11.674 & 0.662 & 1.004 & $2002 \mathrm{Jul} 4$ & $\mathrm{KP} 2.1 \mathrm{~m}$ & M5 & $7.4 \pm 0.5$ & $72 \pm 15$ & 6,7 \\
\hline $1631136+192200 \ldots \ldots .$. & $\ldots$ & 13.457 & 0.724 & 1.052 & $2002 \mathrm{Jul} 4$ & $\mathrm{KP} 2.1 \mathrm{~m}$ & M5 & $9.7 \pm 0.4$ & $56 \pm 11$ & \\
\hline $1734419+123105 \ldots \ldots \ldots$ & LHS 2980 & 13.095 & 0.715 & 1.031 & $2002 \mathrm{Jul} 4$ & $\mathrm{KP} 2.1 \mathrm{~m}$ & M1 & $6.4 \pm 0.1$ & $222 \pm 15$ & 1 \\
\hline $2002066-023314 \ldots \ldots$. & $\ldots$ & 10.502 & 0.779 & 1.093 & $2002 \mathrm{Jul} 5$ & $\mathrm{KP} 2.1 \mathrm{~m}$ & M3 & $7.1 \pm 0.2$ & $48 \pm 4$ & \\
\hline $2003438-144917 \ldots \ldots$. & $\ldots$ & 13.580 & 0.718 & 1.029 & $2002 \mathrm{Jul} 4$ & KP $2.1 \mathrm{~m}$ & M5 & $9.5 \pm 0.2$ & $65 \pm 5$ & \\
\hline $2040269-152316 \ldots \ldots$. & $\ldots$ & 13.656 & 0.761 & 1.089 & $2002 \mathrm{Jul} 4$ & KP $2.1 \mathrm{~m}$ & M2 & $7.2 \pm 0.2$ & $200 \pm 22$ & \\
\hline $2107247-335733 \ldots \ldots$. & DENIS-P J2107-3357 & 12.213 & 0.613 & 1.056 & $2002 \mathrm{Jul} 7$ & KP $2.1 \mathrm{~m}$ & M6 & $10.1 \pm 0.4$ & $26 \pm 5$ & 8,9 \\
\hline $2208546-244911 \ldots \ldots . .$. & $\ldots$ & 12.897 & 0.707 & 1.016 & $2002 \mathrm{Jul} 4$ & $\mathrm{KP} 2.1 \mathrm{~m}$ & M6 & $10.1 \pm 0.4$ & $36 \pm 6$ & \\
\hline $2215171-045919 \ldots \ldots$ & LP 699-64 & 13.441 & 0.688 & 1.004 & $2002 \mathrm{Jul} 4$ & $\mathrm{KP} 2.1 \mathrm{~m}$ & M6 & $10.1 \pm 0.4$ & $46 \pm 8$ & 10 \\
\hline 2229444-192324 _....... & & 13.512 & 0.696 & 1.025 & $2002 \mathrm{Jul} 4$ & $\mathrm{KP} 2.1 \mathrm{~m}$ & M3 & $7.0 \pm 0.1$ & $197 \pm 13$ & \\
\hline $2309142-353159 \ldots \ldots$ & LP 985-98 & 12.035 & 0.684 & 1.049 & 2002 Jul 3 & $\mathrm{KP} 2.1 \mathrm{~m}$ & M5 & $9.5 \pm 0.2$ & $33 \pm 2$ & 10 \\
\hline $2336142-093606 \ldots \ldots$ & $\ldots$ & 13.393 & 0.635 & 1.094 & $2002 \mathrm{Jul} 5$ & $\mathrm{KP} 2.1 \mathrm{~m}$ & M6.5 & $10.5 \pm 0.3$ & $39 \pm 5$ & \\
\hline $2338541-124618 \ldots \ldots \ldots$ & $\ldots$ & 12.181 & 0.557 & 1.014 & $2002 \mathrm{Jul} 5$ & $\mathrm{KP} 2.1 \mathrm{~m}$ & M6.5 & $10.5 \pm 0.3$ & $22 \pm 3$ & \\
\hline $2340477+462318 \ldots \ldots \ldots$ & $\ldots$ & 13.320 & 0.680 & 1.001 & $2002 \mathrm{Jul} 6$ & $\mathrm{KP} 2.1 \mathrm{~m}$ & M6 & $10.1 \pm 0.4$ & $44 \pm 8$ & \\
\hline $2353081-082916 \ldots \ldots$. & LP 763-14 & 13.274 & 0.651 & 1.017 & $2002 \mathrm{Jul} 6$ & $\mathrm{KP} 2.1 \mathrm{~m}$ & M5 & $9.3 \pm 0.4$ & $62 \pm 11$ & 10 \\
\hline
\end{tabular}

Note.-Table 4 is also available in machine-readable form in the electronic edition of the Astronomical Journal.

a The 2MASS designation is 2MASSI Jhhmmss[.]s \pm ddmmss.

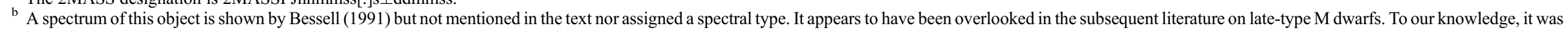
first formally assigned a spectral type by Reid et al. (2003b [Paper VII]) using the same data presented here.

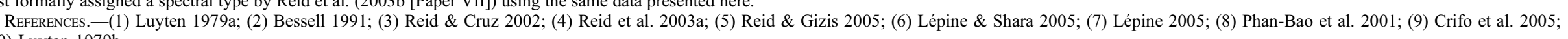
(10) Luyten 1979b. 
TABLE 5

Spectroscopically Confirmed Giants

\begin{tabular}{|c|c|c|c|c|c|c|}
\hline \multirow[b]{2}{*}{ 2MASSI Designation ${ }^{a}$} & \multicolumn{3}{|c|}{ 2MASS } & \multirow[b]{2}{*}{ Obs. Date (UT) } & \multirow[b]{2}{*}{ TELESCOPE } & \multirow[b]{2}{*}{ Optical Spectral Type } \\
\hline & $J$ & $J-H$ & $J-K_{S}$ & & & \\
\hline $0057017+450949$ & 10.002 & 0.776 & 1.202 & 2002 Jul 8 & $\mathrm{KP} 2.1 \mathrm{~m}$ & M8 III \\
\hline $0426258+154502 \ldots \ldots \ldots \ldots \ldots \ldots$ & 11.860 & 0.695 & 1.021 & 2002 Sep 26 & KP 4 m & M5 III \\
\hline $0557509-135950$..................... & 12.873 & 0.741 & 1.135 & 2002 Sep 27 & $\mathrm{KP} 4 \mathrm{~m}$ & M6 pec III ${ }^{\mathrm{b}}$ \\
\hline 0712435+395831 ….................. & 9.156 & 0.743 & 1.119 & 2002 Sep 25 & KP 4 m & K5 III \\
\hline $1119051+700609 \ldots \ldots \ldots \ldots \ldots \ldots . .$. & 10.276 & 0.741 & 1.096 & 2002 Jul 8 & $\mathrm{KP} 2.1 \mathrm{~m}$ & M4 III \\
\hline $1340371-011604 \ldots \ldots \ldots \ldots \ldots \ldots$ & 13.492 & 0.619 & 1.106 & $2002 \mathrm{Jul} 7$ & $\mathrm{KP} 2.1 \mathrm{~m}$ & $<$ K5 III \\
\hline 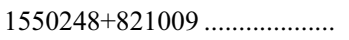 & 9.667 & 0.778 & 1.095 & $2002 \mathrm{Jul} 10$ & APO & M7 III \\
\hline 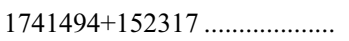 & 9.173 & 0.758 & 1.071 & 2002 Sep 25 & $\mathrm{KP} 4 \mathrm{~m}$ & M3 III \\
\hline $1750217+132703 \ldots \ldots \ldots \ldots \ldots \ldots$ & 9.128 & 0.759 & 1.063 & 2002 Jul 6 & $\mathrm{KP} 2.1 \mathrm{~m}$ & M7 III \\
\hline 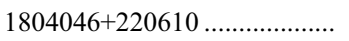 & 9.260 & 0.788 & 1.098 & $2002 \mathrm{Jul} 3$ & $\mathrm{KP} 2.1 \mathrm{~m}$ & M3 III \\
\hline 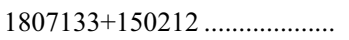 & 9.108 & 0.799 & 1.109 & 2002 Sep 25 & $\mathrm{KP} 4 \mathrm{~m}$ & M3 III \\
\hline 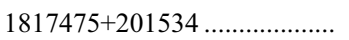 & 9.825 & 0.798 & 1.118 & 2002 Sep 25 & $\mathrm{KP} 4 \mathrm{~m}$ & K5 III \\
\hline 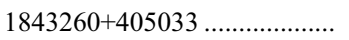 & 9.275 & 0.780 & 1.144 & 2002 Jul 3 & $\mathrm{KP} 2.1 \mathrm{~m}$ & M3 III \\
\hline $1923327-305502$ …................ & 9.222 & 0.756 & 1.083 & 2002 Jul 3 & $\mathrm{KP} 2.1 \mathrm{~m}$ & M5 III \\
\hline 1927415-323251 …................ & 9.008 & 0.769 & 1.109 & 2002 Jul 5 & $\mathrm{KP} 2.1 \mathrm{~m}$ & M5 III \\
\hline 1936154-343109 …................. & 10.837 & 0.780 & 1.111 & 2002 Sep 25 & $\mathrm{KP} 4 \mathrm{~m}$ & M0 III \\
\hline $1941178-262716 \ldots \ldots \ldots \ldots \ldots \ldots . .$. & 10.525 & 0.835 & 1.246 & 2002 Jul 5 & $\mathrm{KP} 2.1 \mathrm{~m}$ & M5 III \\
\hline $1959007-223323 \ldots \ldots \ldots \ldots \ldots \ldots$ & 9.147 & 0.720 & 1.038 & 2002 Jul 5 & $\mathrm{KP} 2.1 \mathrm{~m}$ & M1 III \\
\hline $2000171-270537$..................... & 9.096 & 0.771 & 1.260 & 2002 Jul 5 & $\mathrm{KP} 2.1 \mathrm{~m}$ & M7 III \\
\hline $2002404-294746$...................... & 9.157 & 0.720 & 1.070 & 2003 Jul 9 & $\mathrm{KP} 4 \mathrm{~m}$ & M3 III \\
\hline $2005582-012730$ & 9.314 & 0.915 & 1.481 & 2002 Jul 3 & $\mathrm{KP} 2.1 \mathrm{~m}$ & M6 III \\
\hline $2007596-043924$...................... & 9.576 & 0.914 & 1.456 & 2002 Jul 3 & $\mathrm{KP} 2.1 \mathrm{~m}$ & M5 III \\
\hline 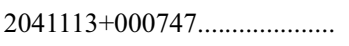 & 9.220 & 0.726 & 1.026 & 2002 Sep 26 & $\mathrm{KP} 4 \mathrm{~m}$ & M0 III \\
\hline $2102375+184551 \ldots \ldots \ldots \ldots \ldots \ldots . .$. & 9.674 & 0.796 & 1.203 & 2003 Apr 20 & CT $4 \mathrm{~m}$ & M7 III \\
\hline 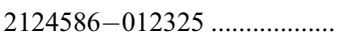 & 9.975 & 0.782 & 1.121 & 2002 Jul 3 & $\mathrm{KP} 2.1 \mathrm{~m}$ & M3 III \\
\hline $2133408+292531 \ldots \ldots \ldots \ldots \ldots \ldots \ldots$ & 9.257 & 0.789 & 1.093 & 2003 Jul 9 & $\mathrm{KP} 4 \mathrm{~m}$ & M0 III \\
\hline 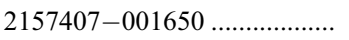 & 13.662 & 0.773 & 1.136 & 2002 Sep 25 & $\mathrm{KP} 4 \mathrm{~m}$ & M4 III \\
\hline 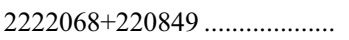 & 9.698 & 0.717 & 1.158 & 2003 Jul 9 & $\mathrm{KP} 4 \mathrm{~m}$ & M8 III \\
\hline $2233559+403935 \ldots \ldots \ldots \ldots \ldots \ldots . .$. & 9.792 & 0.785 & 1.122 & 2003 Jul 9 & $\mathrm{KP} 4 \mathrm{~m}$ & M0 III \\
\hline 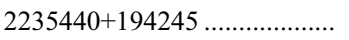 & 13.501 & 0.703 & 1.030 & $2002 \mathrm{Jul} 4$ & $\mathrm{KP} 2.1 \mathrm{~m}$ & K5 III \\
\hline $2237158+372132 \ldots \ldots \ldots \ldots \ldots \ldots$ & 9.006 & 0.788 & 1.111 & $2003 \mathrm{Jul} 10$ & $\mathrm{KP} 4 \mathrm{~m}$ & M3 III \\
\hline $2238182+411355$....................... & 10.260 & 0.800 & 1.100 & 2002 Sep 25 & $\mathrm{KP} 4 \mathrm{~m}$ & M0 III \\
\hline $2350294+451749 \ldots \ldots \ldots \ldots \ldots \ldots \ldots$ & 10.167 & 0.833 & 1.222 & 2002 Jul 6 & $\mathrm{KP} 2.1 \mathrm{~m}$ & M4 III \\
\hline
\end{tabular}

a The 2MASS designation is 2MASSI Jhhmmss[.]s \pm ddmmss.

${ }^{b}$ Spectrum displays $\mathrm{H} \alpha$ in emission.

TABLE 6

Spectroscopically Confirmed Carbon Stars

\begin{tabular}{|c|c|c|c|c|c|}
\hline \multirow[b]{2}{*}{ 2MASSI Designation ${ }^{\mathrm{a}}$} & \multicolumn{3}{|c|}{ 2MASS } & \multirow[b]{2}{*}{ Obs. Date (UT) } & \multirow[b]{2}{*}{ Telescope } \\
\hline & $J$ & $J-H$ & $J-K_{S}$ & & \\
\hline $0008170-753727 \ldots \ldots \ldots \ldots \ldots$ & 13.826 & 1.259 & 2.224 & 2006 Jan 15 & CT $4 \mathrm{~m}$ \\
\hline 0027523-705231 ….............. & 13.368 & 1.382 & 2.320 & 2003 Nov 9 & CT $1.5 \mathrm{~m}$ \\
\hline 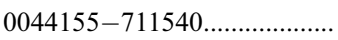 & 13.398 & 1.189 & 2.006 & 2003 Nov 9 & CT $1.5 \mathrm{~m}$ \\
\hline $0046008-752112 \ldots \ldots \ldots \ldots \ldots \ldots$ & 13.182 & 1.071 & 1.922 & 2003 Nov 9 & CT $1.5 \mathrm{~m}$ \\
\hline $0107522-692136 \ldots \ldots \ldots \ldots \ldots \ldots$ & 13.153 & 1.288 & 2.177 & 2003 Nov 9 & CT $1.5 \mathrm{~m}$ \\
\hline $0121433-740346 \ldots \ldots \ldots \ldots \ldots \ldots$ & 13.688 & 1.488 & 2.604 & 2006 Jan 15 & $\mathrm{CT} 4 \mathrm{~m}$ \\
\hline $0126348-703947$...................... & 12.817 & 1.478 & 2.494 & 2003 Nov 9 & CT $1.5 \mathrm{~m}$ \\
\hline $0148078-715521 \ldots \ldots \ldots \ldots \ldots \ldots$ & 12.471 & 1.271 & 2.199 & 2003 Nov 10 & CT $1.5 \mathrm{~m}$ \\
\hline $0156260+512521 \ldots \ldots \ldots \ldots \ldots \ldots$ & 9.395 & 1.143 & 1.713 & 2003 Jul 9 & $\mathrm{KP} 4 \mathrm{~m}$ \\
\hline 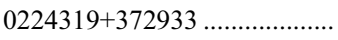 & 11.498 & 1.619 & 2.775 & $2003 \mathrm{Jul} 9$ & $\mathrm{KP} 4 \mathrm{~m}$ \\
\hline 0554397-144658 ...................... & 12.731 & 0.767 & 1.083 & 2002 Sep 27 & $\mathrm{KP} 4 \mathrm{~m}$ \\
\hline 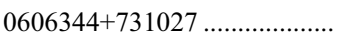 & 9.397 & 0.986 & 1.519 & 2002 Sep 25 & $\mathrm{KP} 4 \mathrm{~m}$ \\
\hline 1015259-020431 ….............. & 14.045 & 1.184 & 2.058 & 2003 Apr 22 & CT $4 \mathrm{~m}$ \\
\hline $1504553+354757 \ldots \ldots \ldots \ldots \ldots \ldots$ & 12.000 & 1.322 & 2.323 & 2003 Mar 14 & $\mathrm{KP} 2.1 \mathrm{~m}$ \\
\hline $1941285-323338$...................... & 10.865 & 0.959 & 1.412 & 2002 Sep 25 & $\mathrm{KP} 4 \mathrm{~m}$ \\
\hline $2002292-245258 \ldots \ldots \ldots \ldots \ldots \ldots$ & 9.199 & 0.774 & 1.077 & $2002 \mathrm{Jul} 3$ & $\mathrm{KP} 2.1 \mathrm{~m}$ \\
\hline $2252361+474125 \ldots \ldots \ldots \ldots \ldots \ldots$ & 9.624 & 1.073 & 1.633 & $2003 \mathrm{Jul} 9$ & $\mathrm{KP} 4 \mathrm{~m}$ \\
\hline
\end{tabular}

${ }^{\text {a }}$ The 2MASS designation is 2MASSI Jhhmmss[.]s \pm ddmmss. 
TABLE 7

Objects with New Spectra

\begin{tabular}{|c|c|c|c|c|c|c|c|c|c|c|c|}
\hline \multirow[b]{2}{*}{ 2MASSI Designation ${ }^{\mathrm{a}}$} & \multirow[b]{2}{*}{ 2MUCD No. } & \multirow[b]{2}{*}{ OtHER NAMES } & \multicolumn{3}{|c|}{ 2MASS } & \multirow[b]{2}{*}{ OBs. Date (UT) } & \multirow[b]{2}{*}{ Telescope } & \multirow[b]{2}{*}{ Optical Spectral Type } & \multirow[b]{2}{*}{$M_{J}$} & \multirow{2}{*}{$\begin{array}{c}d \\
(\mathrm{pc})\end{array}$} & \multirow[b]{2}{*}{ Other ReFs } \\
\hline & & & $J$ & $J-H$ & $J-K_{S}$ & & & & & & \\
\hline $0141032+180450 \ldots \ldots \ldots$ & 10085 & $\ldots$ & 13.822 & 0.772 & 1.315 & 2004 Feb 11 & $\mathrm{KP} 4 \mathrm{~m}$ & L1 & $12.00 \pm 0.14$ & $23.1 \pm 1.6$ & 1 \\
\hline $0326422-210205 \ldots \ldots$. & 10184 & $\ldots$ & 16.111 & 1.337 & 2.226 & 2005 Oct 10 & GS & $\mathrm{L} 4^{\mathrm{b}}$ & $13.09 \pm 0.22$ & $40.1 \pm 4.5$ & 2 \\
\hline $0428509-225322 \ldots \ldots$ & 10286 & $\ldots$ & 13.579 & 0.882 & 1.459 & 2004 Feb 11 & KP $4 \mathrm{~m}$ & L1 & $12.00 \pm 0.14$ & $20.7 \pm 1.4$ & 3 \\
\hline $1003191-010507 \ldots \ldots$. & 10873 & LHS 5165/DENIS-P J1003-0105 & 12.352 & 0.667 & 1.085 & 2003 Mar 15 & $\mathrm{KP} 2.1 \mathrm{~m}$ & M7 & $10.73 \pm 0.25$ & $21.1 \pm 2.5$ & 4,5 \\
\hline $1011002+424503 \ldots \ldots \ldots$ & 10885 & TVLM 263-71765 & 13.357 & 0.657 & 1.056 & 2004 Feb 10 & KP 4 m & M7.5 & $10.96 \pm 0.21$ & $30.1 \pm 2.9$ & 6 \\
\hline $1515009+484739 \ldots \ldots .$. & 11314 & $\ldots$ & 14.060 & 0.991 & 1.495 & $\begin{array}{l}2002 \text { Jul } 10 \\
2004 \text { Feb } 10\end{array}$ & $\begin{array}{l}\text { APO } \\
\text { KP } 4 \text { m }\end{array}$ & L6 & $14.02 \pm 0.30$ & $10.2 \pm 1.4$ & 1 \\
\hline
\end{tabular}

a The 2MASS designation is 2MASSI Jhhmmss[.]s \pm ddmmss.

. 2 and discussed in $\S 5.1$

References.-(1) Wilson 2002; (2) Cruz et al. 2003; (3) Kendall et al. 2003; (4) Gizis 2002; (5) Phan-Bao et al. 2001; (6) Tinney et al. 1995.

TABLE 8

Low-Gravity Objects

\begin{tabular}{|c|c|c|c|c|c|c|c|c|c|c|c|c|}
\hline \multirow[b]{2}{*}{ 2MASSI DeSIGNATION ${ }^{\mathrm{a}}$} & \multirow[b]{2}{*}{ 2MUCD No. } & \multirow[b]{2}{*}{ Other NAME } & \multicolumn{3}{|c|}{ 2MASS } & \multirow[b]{2}{*}{ Obs. Date (UT) } & \multirow[b]{2}{*}{ TELESCOPE } & \multirow{2}{*}{$\begin{array}{c}\text { Optical } \\
\text { Spectral Type }\end{array}$} & \multirow{2}{*}{$\begin{array}{c}\mu \\
\left(\text { mas yr }^{-1}\right)\end{array}$} & \multirow{2}{*}{$\begin{array}{l}\text { Position ANGLE } \\
\text { (deg) }\end{array}$} & \multirow[b]{2}{*}{$\mu$ ReF. } & \multirow[b]{2}{*}{ OTHER REFS } \\
\hline & & & $J$ & $J-H$ & $J-K_{S}$ & & & & & & & \\
\hline $0241115-032658 \ldots \ldots \ldots \ldots \ldots \ldots \ldots$ & 10141 & $\ldots$ & 15.831 & 1.019 & 1.807 & $\begin{array}{l}2002 \text { Sep } 27 \\
2005 \text { Oct } 10\end{array}$ & $\begin{array}{l}\text { KP } 4 \mathrm{~m} \\
\text { GS }\end{array}$ & (L1) & $57 \pm 18$ & 88 & 1 & \\
\hline $0436278-411446 \ldots \ldots \ldots \ldots \ldots \ldots \ldots$ & 10306 & DENIS-P J0436-4114 & 13.105 & 0.689 & 1.043 & $\begin{array}{l}2003 \text { Nov } 8 \\
2006 \text { Jan } 15\end{array}$ & $\begin{array}{l}\text { CT } 1.5 \mathrm{~m} \\
\text { CT } 4 \mathrm{~m}\end{array}$ & $(\mathrm{M} 8)^{\mathrm{c}}$ & $22 \pm 18$ & 80 & 2 & 1,2 \\
\hline $0443376+000205 \ldots \ldots \ldots \ldots \ldots \ldots$ & 10320 & SDSS $0443+0002$ & 12.517 & 0.713 & 1.350 & 2002 Jan 28 & CT $1.5 \mathrm{~m}$ & $(\mathrm{M} 9)^{\mathrm{d}}$ & $159 \pm 78$ & 146 & 1 & 3,4 \\
\hline $1615425+495321$ & 11538 & $\ldots$ & 16.732 & 1.441 & 2.413 & 2004 Sep 12 & GN & (L4) & $115 \pm 83$ & 287 & 1 & \\
\hline $2213449-213607$.......................... & 12120 & $\ldots$ & 15.392 & 0.975 & 1.647 & $\begin{array}{l}2002 \text { Sep } 27 \\
2005 \text { Sep } 8\end{array}$ & $\begin{array}{l}\text { KP } 4 \mathrm{~m} \\
\text { GS }\end{array}$ & (L1) & $\cdots$ & $\cdots$ & & \\
\hline
\end{tabular}

Note. - Spectra are plotted in Figs. 4-7.

The 2MASS designation is 2MASSI Jhhmmss[.]s \pm ddmmss.

The spectral types should be regarded as preliminary since all of these objects have low-gravity spectral features and do not fit into the dwarf spectral sequence as it is currently defined.

c These new spectral data supplement the photometric data of Phan-Bao et al. (2003) listed in Paper V.

d This is a reclassification based on a reanalysis of the spectrum presented in Paper V.

References.-Proper motion: (1) This paper; (2) Phan-Bao et al. 2003. Other: (1) Phan-Bao et al. 2003; (2) Crifo et al. 2005; (3) Wilson 2002 ; (4) Hawley et al. 2002. 
J143517.20-004612.9 (L0), and SDSS J225529.09-003433.4 (L0:). PC $0025+0447$ is recognized as a highly unusual object that is young, has low-gravity features, and has persistent, extremely strong $\mathrm{H} \alpha$ emission $(\mathrm{EW} \sim 100 \AA$ ). The two SDSS objects are faint $(J>15.6)$ and the photometric errors in SDSS 1435-00 are substantial $(\sim 0.1)$. We propose that either these three objects are unusual in a similar as-yet-unrecognized way, or, more likely, that the uncertainties in the 2MASS photometry, due to either systematic errors or coincidence, have resulted in a similar blue $\left(H-K_{S}\right)$ color. No correction is applied to the luminosity functions based on these three objects.

\subsubsection{Other Excluded Objects}

Two additional objects in the UCDt sample are excluded by the $(J-H) /\left(H-K_{S}\right)$ selection criteria: GJ 1048B (L1) with $(J-H)=0.69,\left(H-K_{S}\right)=0.67$, and SDSS J144600.60+ 002452.0 (L6) with $(J-H)=1.38,\left(H-K_{S}\right)=0.58$. The $\left(H-K_{S}\right)$ color of GJ $1048 \mathrm{~B}$ is unusually red for its spectral type and is probably due to its proximity to GJ 1048A, resulting in $\sim 0.1$ mag photometric uncertainties. For an L6, SDSS 1446+00 appears unusually red in $(J-H)$, and the 2MASS photometry is fairly robust, with uncertainties ranging from 0.035 in the $H$ band to $0.082 \mathrm{mag}$ in the $J$ band. However, the 2MASS flag $n$ det equals 264566 , indicating that there were only two detections at the $J$ band out of six possible, four detections out of five possible at the $H$ band, and six out of six at the $K_{S}$ band. Thus, with only two detections, the quoted $J$ magnitude is highly suspect. Using the transformation given in the 2MASS Explanatory Supplement ${ }^{24}$ we convert the UKIRT MKO photometry listed in Geballe et al. (2002) to the 2MASS system and find $J H K_{S}$ colors that are not anomalous when compared to other late- $\mathrm{L}$ dwarfs. A more accurate 2MASS $J$ magnitude is probably 15.6 rather than the 15.9 listed in both the 2MASS Second Release PSC and the All-Sky PSC. No correction is applied to the luminosity functions based on these two objects.

\subsubsection{Volume Completeness}

We show the space densities for two sets of 4 pc thick spherical shells for five absolute magnitude bins in Figure 15. [Note that these bins are coarser than those used in $\Phi\left(M_{J}\right)$ and $\Phi\left(M_{K_{S}}\right)$, and the last two bins overlap.] The distance at which the sample begins to be incomplete is indicated by a downturn in the measured space densities. The $2 \mathrm{MU} 2$ sample appears to be complete to $20 \mathrm{pc}$ at all magnitudes except for the two faintest $(14<$ $\left.M_{J}<15\right)$. This is likely due to our incompleteness at the latest L types, and, as discussed above, we take our measurement of the luminosity functions to be a lower limit at the relevant magnitudes.

\subsection{Malmquist Bias}

We have adopted a unique value of $M_{J}$ for each spectral type, $M_{\mathrm{obs}}$, for which the true situation is a dispersion of absolute magnitudes about some average for each spectral type. As a result, the sample is biased toward more luminous objects at a given spectral type. The intrinsically less luminous objects (where the true intrinsic absolute magnitude $M_{0}>M_{\text {obs }}$ ) are systematically excluded because using $M_{\mathrm{obs}}$ overestimates their distance, and, thus, they are more likely to fall outside of the distance limit. Similarly, a greater number of overluminous objects are included in the sample because their distances are underestimated. This is classical Malmquist bias, and we must correct the estimated

\footnotetext{
${ }^{24}$ Available at http://www.ipac.caltech.edu/2mass/releases/allsky/doc/sec6_ 4b.html.
}

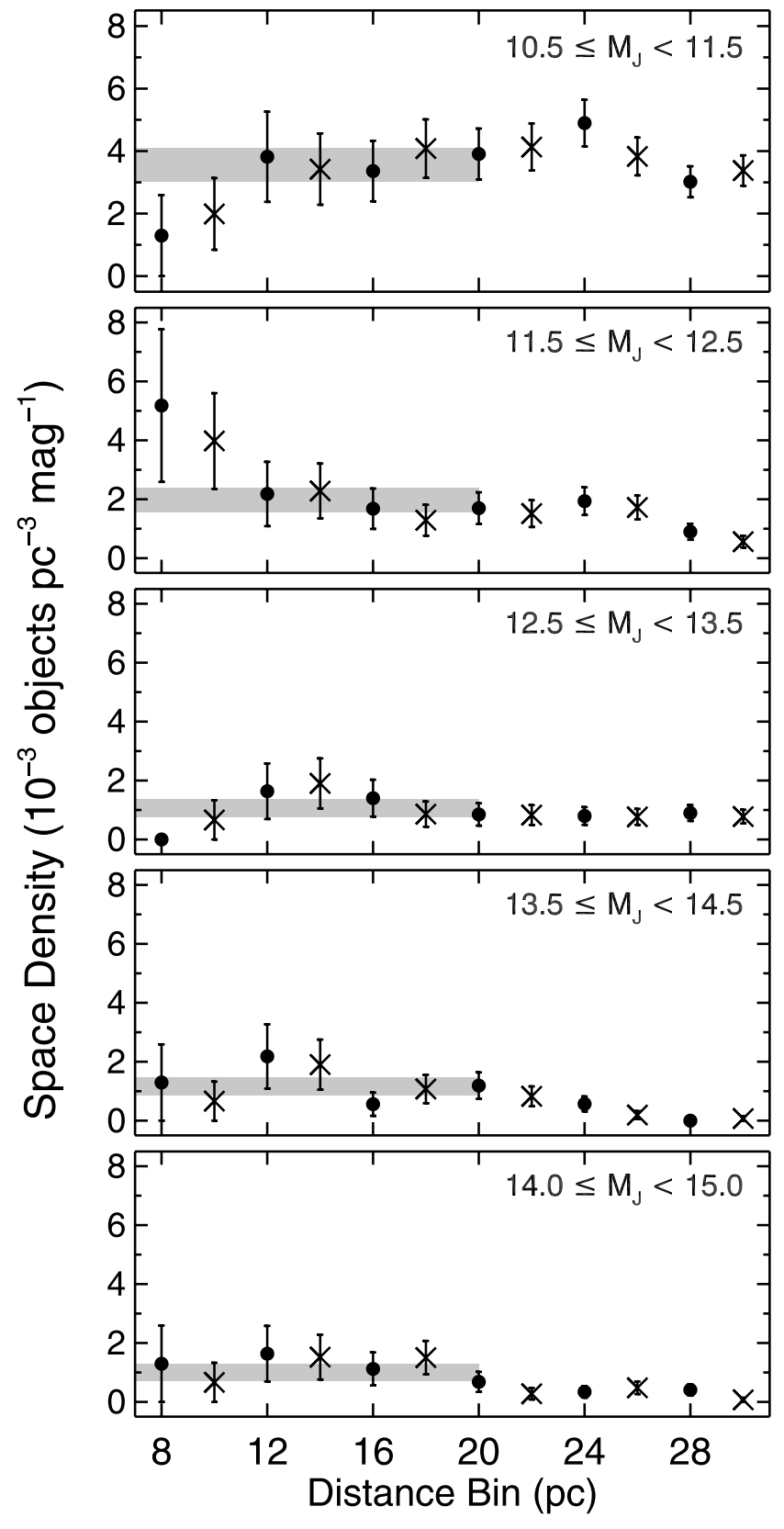

FIG. 15.- Malmquist-corrected space densities for two sets of spherical shells for the 2MU2 sample as a function of $M_{J}$. The densities are shown for spherical shells with inner and outer radii of $0-8,8-12,12-16,16-20,20-24$, and 24$28 \mathrm{pc}$ (circles) and $0-10,10-14,14-18,18-22,22-26$, and 26-30 pc (crosses). The density for each shell is plotted at the distance of the outer radius (e.g., the density for the $8-12 \mathrm{pc}$ shell is plotted at $12 \mathrm{pc}$ ). The shaded bar indicates the overall density for each $M_{J}$ bin and the associated Poisson uncertainty. These $M_{J}$ bins are coarser than those used in $\Phi\left(M_{J}\right)$ and $\Phi\left(M_{K_{S}}\right), 1$ mag wide instead of $0.5 \mathrm{mag}$, and the two faintest overlap due to the odd number of bins.

absolute magnitude for those 69 objects in the sample without trigonometric parallax data (Malmquist 1920).

Since we expect nearby M and L dwarfs to be uniformly distributed throughout the solar neighborhood, we can use Malmquist's formula to correct the absolute magnitudes,

$$
M_{0}=M_{\mathrm{obs}}-1.38 \sigma^{2}
$$

where $\sigma$ is the uncertainty in $M_{\mathrm{obs}}$. The uncertainty in $M_{\mathrm{obs}}$ depends partly on the scatter in the $M_{J}$-spectral-type relation but mostly on the uncertainties in the spectral-type estimates. Combining these, 
TABLE 9

The 20 pe 2MU2 Sample Used to Estimate the Luminosity Function

\begin{tabular}{|c|c|c|c|c|c|c|c|c|c|c|c|c|}
\hline \multirow[b]{2}{*}{ 2MASSI Designation ${ }^{\mathrm{a}}$} & \multirow[b]{2}{*}{ 2MUCD No. } & \multirow[b]{2}{*}{ OTHER NAMES } & \multicolumn{3}{|c|}{ 2MASS } & \multirow{2}{*}{$\begin{array}{c}\text { Optical } \\
\text { SPeCtral TyPe }^{\mathrm{b}}\end{array}$} & \multirow[b]{2}{*}{$M_{J}$} & \multirow[b]{2}{*}{$M_{K_{S}}$} & \multirow{2}{*}{$\begin{array}{c}d \\
(\mathrm{pc})^{\mathrm{c}}\end{array}$} & \multirow{2}{*}{$\begin{array}{c}d \\
\text { SourCE }^{\mathrm{c}}\end{array}$} & \multirow{2}{*}{$\begin{array}{l}\text { Discovery } \\
\text { Refs. }\end{array}$} & \multirow{2}{*}{$\begin{array}{l}\text { Parallax } \\
\text { Refs. }\end{array}$} \\
\hline & & & $J$ & $J-H$ & $J-K_{S}$ & & & & & & & \\
\hline $0019262+461407 \ldots \ldots \ldots \ldots . .$. & 10013 & $\ldots$ & 12.609 & 0.676 & 1.135 & M8 & $11.16 \pm 0.18$ & $10.02 \pm 0.18$ & $19.5 \pm 1.6$ & ST & 1 & \\
\hline $0019457+521317 \ldots \ldots \ldots \ldots . .$. & 10014 & & 12.820 & 0.748 & 1.204 & M9 & $11.47 \pm 0.14$ & $10.26 \pm 0.15$ & $18.7 \pm 1.2$ & ST & 1 & \\
\hline $0024246-015819 \ldots \ldots \ldots \ldots . .$. & 10018 & BRI $0021-0214$ & 11.860 & 0.740 & 1.280 & M9.5 & $11.55 \pm 0.10$ & $10.27 \pm 0.10$ & $11.55 \pm 0.53$ & Parallax & 2 & 1 \\
\hline $0024442-270825 B \ldots \ldots \ldots \ldots$. & 10019 & LHS $1070 \mathrm{~B}^{\mathrm{d}}$ & 9.262 & 0.730 & 1.030 & M8.5 & $11.64 \pm 0.06$ & $10.49 \pm 0.05$ & $7.71 \pm 0.14$ & Parallax & $3,4,5$ & 2,3 \\
\hline $0024442-270825 \mathrm{C} \ldots \ldots \ldots \ldots$ & $\ldots$ & LHS $1070 \mathrm{C}$ & $\ldots$ & $\ldots$ & $\ldots$ & L0 & $11.97 \pm 0.08$ & $10.81 \pm 0.07$ & $7.71 \pm 0.14$ & Parallax & $3,4,5$ & 2,3 \\
\hline $0027559+221932 \mathrm{~A} \ldots \ldots \ldots \ldots$ & 10022 & LP $349-25 A$ & 10.608 & 0.638 & 1.047 & M8: & $11.16 \pm 0.35$ & $10.13 \pm 0.24$ & $10.3 \pm 1.7$ & ST & 6,7 & \\
\hline $0027559+221932 \mathrm{~B} \ldots \ldots \ldots \ldots$ & & LP 349-25B & & & & M9: & $11.47 \pm 0.28$ & $10.39 \pm 0.25$ & $10.3 \pm 1.7$ & ST & 8 & \\
\hline $0109511-034326 \ldots \ldots \ldots \ldots . .$. & 10068 & LP $647-13$ & 11.695 & 0.774 & 1.277 & M9 & $11.47 \pm 0.14$ & $10.51 \pm 0.06$ & $11.1 \pm 0.7$ & ST & $1,6,9$ & \\
\hline $0123112-692138 \ldots \ldots \ldots \ldots . .$. & 13056 & $\ldots$ & 12.335 & 0.619 & 1.034 & M8 & $11.16 \pm 0.18$ & $10.12 \pm 0.18$ & $17.2 \pm 1.4$ & ST & 10 & \\
\hline $0144353-071614 \ldots \ldots \ldots \ldots . .$. & 10088 & $\ldots$ & 14.187 & 1.183 & 1.904 & L5 & $13.55 \pm 0.24$ & $11.65 \pm 0.24$ & $13.4 \pm 1.5$ & ST & $1,11,12$ & \\
\hline $0148386-302439 \ldots \ldots \ldots \ldots . .$. & 10091 & $\ldots$ & 12.282 & 0.641 & 1.038 & M7.5 & $10.96 \pm 0.21$ & $9.93 \pm 0.21$ & $18.4 \pm 1.8$ & ST & 1 & \\
\hline $0205293-115930 \mathrm{~A}^{\mathrm{e}} \ldots \ldots \ldots$. & 10096 & DENIS-P J0205.4-1159A & 14.581 & 0.991 & 1.599 & L7: ${ }^{\mathrm{e}}$ & $13.85 \pm 0.07$ & $12.26 \pm 0.13$ & $19.8 \pm 0.6$ & Parallax & 13 & 4 \\
\hline $0205293-115930 \mathrm{~B} \ldots \ldots \ldots \ldots$. & & DENIS-P J0205.4-1159B & & & & L7: ${ }^{\mathrm{e}}$ & $13.85 \pm 0.07$ & $12.26 \pm 0.16$ & $19.8 \pm 0.6$ & Parallax & 14 & 4 \\
\hline $0213288+444445 \ldots \ldots \ldots \ldots . .$. & 10102 & & 13.512 & 0.740 & 1.269 & $\mathrm{~L} 1.5$ & $12.15 \pm 0.15$ & $10.88 \pm 0.16$ & $18.7 \pm 1.4$ & $\mathrm{ST}$ & 1 & \\
\hline $0248410-165121 \ldots \ldots \ldots \ldots . .$. & 10149 & LP 771-21/BR 0246-1703 & 12.557 & 0.701 & 1.148 & M8 & $11.50 \pm 0.19$ & $10.36 \pm 0.19$ & $16.23 \pm 1.42$ & Parallax & 6,15 & 5 \\
\hline $0251148-035245 \ldots \ldots \ldots \ldots . .$. & 10151 & $\ldots$ & 13.082 & 0.821 & 1.429 & L3 & $12.67 \pm 0.20$ & $11.24 \pm 0.20$ & $12.1 \pm 1.1$ & ST & 1,16 & \\
\hline $0255035-470050 \ldots \ldots \ldots \ldots$ & 10158 & DENIS-P J0255-4700 & 13.225 & 1.036 & 1.698 & L8 & $14.77 \pm 0.30$ & $13.07 \pm 0.30$ & $4.9 \pm 0.3$ & ST & 17,18 & \\
\hline $0318540-342129 \ldots \ldots \ldots \ldots$ & 10176 & & 15.530 & 1.221 & 2.055 & L7 & $14.45 \pm 0.30$ & $12.39 \pm 0.31$ & $16.5 \pm 2.3$ & ST & 18 & \\
\hline $0320596+185423 \ldots \ldots \ldots \ldots \ldots$ & 10179 & LP $412-31$ & 11.744 & 0.701 & 1.172 & $M 8^{f}$ & $10.93 \pm 0.04$ & $9.76 \pm 0.04$ & $14.51 \pm 0.13$ & Parallax & 6,19 & 3,4 \\
\hline $0331302-304238 \ldots \ldots \ldots \ldots . .$. & 10186 & LP $888-18$ & 11.371 & 0.672 & 1.095 & M7.5 & $10.96 \pm 0.21$ & $9.87 \pm 0.21$ & $12.1 \pm 1.2$ & $\mathrm{ST}$ & $1,6,9$ & \\
\hline $0339352-352544 \ldots \ldots \ldots \ldots . .$. & 10201 & LP 944-20/BRI 0337-3535 & 10.748 & 0.731 & 1.223 & M9 & $12.27 \pm 0.05$ & $11.05 \pm 0.05$ & $4.97 \pm 0.10$ & Parallax & 6 & 5 \\
\hline $0340094-672405 \ldots \ldots \ldots \ldots . .$. & 10202 & $\ldots$ & 14.740 & 1.172 & 1.799 & L7:: & $14.45 \pm 0.90$ & $12.65 \pm 0.90$ & $11.4 \pm 4.7$ & ST & 10 & \\
\hline $0351000-005244^{g} \ldots \ldots \ldots \ldots$ & 10213 & LHS 1604 & 11.262 & 0.670 & 1.071 & M7.5 & $10.43 \pm 0.06^{\mathrm{g}}$ & $9.36 \pm 0.06$ & $14.66 \pm 0.39$ & Parallax & $1,3,20$ & 2 \\
\hline $0417374-080000 \ldots \ldots \ldots \ldots . . .$. & 10261 & & 12.166 & 0.654 & 1.112 & M7.5 & $10.96 \pm 0.21$ & $9.85 \pm 0.21$ & $17.4 \pm 1.7$ & ST & 1 & \\
\hline $0423485-041403^{\mathrm{h}} \ldots \ldots \ldots \ldots$ & 10276 & SDSS J0423-0414 ${ }^{\mathrm{h}}$ & 14.452 & 1.010 & 1.516 & L7: ${ }^{\mathrm{h}}$ & $14.04 \pm 0.07$ & $12.45 \pm 0.12$ & $15.17 \pm 0.39$ & Parallax & 21 & 6 \\
\hline 0429184-312356А ............ & 10287 & $\ldots$ & 10.887 & 0.680 & 1.086 & M7.5: & $10.96 \pm 0.42$ & $9.89 \pm 0.32$ & $11.4 \pm 1.1$ & ST & 1 & \\
\hline $0429184-312356 \mathrm{~B} \ldots \ldots \ldots \ldots . .$. & $\ldots$ & $\ldots$ & $\ldots$ & $\ldots$ & $\ldots$ & L1: & $12.00 \pm 0.29$ & $10.87 \pm 0.33$ & $11.4 \pm 1.1$ & ST & 22,23 & \\
\hline $0435161-160657 \ldots \ldots \ldots \ldots . .$. & 10302 & LP $775-31$ & 10.396 & 0.616 & 1.060 & M7 & $10.73 \pm 0.25$ & $9.67 \pm 0.26$ & $8.6 \pm 1.0$ & ST & $1,6,9$ & \\
\hline $0439010-235308 \ldots \ldots \ldots \ldots . .$. & 10312 & $\ldots$ & 14.413 & 1.045 & 1.606 & L6.5 & $14.24 \pm 0.30$ & $12.64 \pm 0.30$ & $10.8 \pm 1.5$ & ST & 1 & \\
\hline $0440232-053008 \ldots \ldots \ldots \ldots . .$. & 10316 & LP $655-48$ & 10.681 & 0.696 & 1.124 & M7 & $10.73 \pm 0.25$ & $9.61 \pm 0.26$ & $9.8 \pm 1.1$ & ST & $1,6,9$ & \\
\hline $0443376+000205 \ldots \ldots \ldots \ldots . .$. & 10320 & SDSS $0443+0002$ & 12.517 & 0.713 & 1.350 & $(\mathrm{M} 9)^{\mathrm{i}}$ & $11.47 \pm 0.28$ & $10.12 \pm 0.28$ & $16.2 \pm 2.1$ & ST & 16,24 & \\
\hline $0445538-304820 \ldots \ldots \ldots \ldots . .$. & 10329 & & 13.409 & 0.835 & 1.425 & $\mathrm{~L} 2$ & $12.31 \pm 0.17$ & $10.88 \pm 0.17$ & $16.6 \pm 1.3$ & ST & 1 & \\
\hline $0517376-334902 \ldots \ldots \ldots \ldots . .$. & 10380 & DENIS-P J0517-3349 & 11.995 & 0.672 & 1.176 & M8 & $11.16 \pm 0.18$ & $9.98 \pm 0.18$ & $14.7 \pm 1.2$ & ST & 1,25 & \\
\hline $0523382-140302 \ldots \ldots \ldots \ldots . .$. & 10390 & $\ldots$ & 13.117 & 0.896 & 1.486 & $\mathrm{~L} 2.5$ & $12.48 \pm 0.18$ & $11.00 \pm 0.19$ & $13.4 \pm 1.1$ & ST & 1,16 & \\
\hline $0544115-243301 \ldots \ldots \ldots \ldots . .$. & 10444 & $\ldots$ & 12.518 & 0.656 & 1.079 & M8 & $11.16 \pm 0.18$ & $10.08 \pm 0.18$ & $18.7 \pm 1.6$ & ST & 10 & \\
\hline $0652307+471034 \ldots \ldots \ldots \ldots . .$. & 10601 & $\ldots$ & 13.545 & 1.175 & 1.858 & L4.5 & $13.32 \pm 0.23$ & $11.46 \pm 0.24$ & $11.1 \pm 1.2$ & ST & 1 & \\
\hline $0700366+315726 \mathrm{~A} \ldots \ldots \ldots \ldots . . .$. & 10617 & $\ldots$ & 12.922 & 0.967 & 1.614 & L3: & $12.80 \pm 0.06$ & $11.28 \pm 0.22$ & $12.20 \pm 0.30$ & Parallax & 26 & 7 \\
\hline $0700366+315726 \mathrm{~B} \ldots \ldots \ldots \ldots$ & $\ldots$ & $\ldots$ & $\ldots$ & $\ldots$ & $\ldots$ & L6: & $14.00 \pm 0.06$ & $12.16 \pm 0.53$ & $12.20 \pm 0.30$ & Parallax & 23 & 7 \\
\hline $0741068+173845 \ldots \ldots \ldots \ldots . .$. & 10666 & LHS 1937 & 11.995 & 0.633 & 1.026 & M7 & $10.73 \pm 0.25$ & $9.71 \pm 0.26$ & $17.9 \pm 2.1$ & ST & 3,27 & \\
\hline $0746425+200032 \mathrm{~A} \ldots \ldots \ldots \ldots$ & 10668 & $\ldots$ & 11.742 & 0.743 & 1.255 & L0.5: & $11.82 \pm 0.03$ & $10.61 \pm 0.11$ & $12.21 \pm 0.04$ & Parallax & 28 & 4 \\
\hline $0746425+200032 \mathrm{~B} \ldots \ldots \ldots \ldots$ & $\ldots$ & $\ldots$ & $\ldots$ & $\ldots$ & $\ldots$ & L2: & $12.36 \pm 0.04$ & $11.05 \pm 0.18$ & $12.21 \pm 0.04$ & Parallax & 29 & 4 \\
\hline $0752239+161215 \ldots \ldots \ldots \ldots . .$. & 10673 & LP 423-31 & 10.831 & 0.639 & 1.012 & M7 & $10.73 \pm 0.25$ & $9.72 \pm 0.26$ & $10.5 \pm 1.2$ & ST & 1,6 & \\
\hline $0818580+233352 \ldots \ldots \ldots \ldots \ldots$ & 10710 & $\ldots$ & 12.137 & 0.635 & 1.007 & M7 & $10.73 \pm 0.25$ & $9.73 \pm 0.26$ & $19.1 \pm 2.2$ & ST & 7 & \\
\hline $0825196+211552 \ldots \ldots \ldots \ldots . .$. & 10721 & $\ldots$ & 15.116 & 1.328 & 2.071 & L7.5 & $14.98 \pm 0.05$ & $12.91 \pm 0.04$ & $10.66 \pm 0.11$ & Parallax & 28 & 4 \\
\hline
\end{tabular}


TABLE 9-Continued

\begin{tabular}{|c|c|c|c|c|c|c|c|c|c|c|c|c|}
\hline \multirow[b]{2}{*}{ 2MASSI Designation ${ }^{a}$} & \multirow[b]{2}{*}{ 2MUCD No. } & \multirow[b]{2}{*}{ Other Names } & \multicolumn{3}{|c|}{ 2MASS } & \multirow{2}{*}{$\begin{array}{c}\text { Optical } \\
\text { SPECTRAL Type }\end{array}$} & \multirow[b]{2}{*}{$M_{J}$} & \multirow[b]{2}{*}{$M_{K_{S}}$} & \multirow{2}{*}{$\begin{array}{c}d \\
(\mathrm{pc})^{\mathrm{c}}\end{array}$} & \multirow{2}{*}{$\begin{array}{c}d \\
\text { SoURCE }^{\mathrm{c}}\end{array}$} & \multirow{2}{*}{$\begin{array}{l}\text { Discovery } \\
\text { Refs. }\end{array}$} & \multirow{2}{*}{$\begin{array}{c}\text { Parallax } \\
\text { Refs. }\end{array}$} \\
\hline & & & $J$ & $J-H$ & $J-K_{S}$ & & & & & & & \\
\hline 0835425-081923 …........... & 10742 & $\ldots$ & 13.149 & 1.195 & 1.993 & L5 & $13.55 \pm 0.24$ & $11.56 \pm 0.24$ & $8.3 \pm 0.9$ & ST & 1 & \\
\hline 0847287-153237 ................ & 10764 & & 13.519 & 0.892 & 1.465 & L2 & $12.31 \pm 0.17$ & $10.84 \pm 0.17$ & $17.5 \pm 1.4$ & ST & 1 & \\
\hline 0853362-032932 ….............. & 10776 & LHS 2065 & 11.185 & 0.717 & 1.213 & M9 & $11.51 \pm 0.04$ & $10.29 \pm 0.04$ & $8.53 \pm 0.11$ & Parallax & 3,30 & 3,8 \\
\hline 0859254-194926 .................... & 10789 & & 15.505 & 1.067 & 1.778 & $L 7:^{j}$ & $14.45 \pm 0.50$ & $12.67 \pm 0.51$ & $16.3 \pm 3.8$ & ST & 1 & \\
\hline 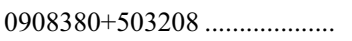 & 10802 & & 14.564 & 1.098 & 1.646 & $\mathrm{~L} 7^{\mathrm{j}}$ & $14.45 \pm 0.30$ & $12.80 \pm 0.30$ & $10.5 \pm 1.5$ & ST & 1,31 & \\
\hline $1006319-165326 \ldots \ldots \ldots \ldots \ldots \ldots .$. & 10877 & LP 789-23 & 12.041 & 0.620 & 1.041 & M7.5 & $10.96 \pm 0.21$ & $9.92 \pm 0.21$ & $16.4 \pm 1.6$ & ST & $1,6,25$ & \\
\hline $1010148-040649$ ………........ & 10880 & & 15.503 & 1.108 & 1.908 & $\mathrm{~L}^{\mathrm{j}}$ & $14.45 \pm 0.30$ & $12.54 \pm 0.31$ & $16.2 \pm 2.3$ & ST & 1 & \\
\hline $1016347+275149$ & 10892 & LHS 2243 & 11.951 & 0.657 & 1.005 & M8 & $11.16 \pm 0.18$ & $10.15 \pm 0.18$ & $14.4 \pm 1.2$ & ST & 3,19 & \\
\hline $1024099+181553$ & 10906 & & 12.242 & 0.664 & 1.037 & M8 & $11.16 \pm 0.18$ & $10.12 \pm 0.18$ & $16.5 \pm 1.4$ & ST & 7 & \\
\hline $1043075+222523$ & 10926 & & 15.947 & 1.202 & 1.956 & L8 & $14.77 \pm 0.50$ & $12.81 \pm 0.51$ & $17.2 \pm 4.0$ & ST & 10 & \\
\hline $1045240-014957$...................... & 10929 & SDSS J1045-0149 & 13.129 & 0.759 & 1.319 & L1 & $12.00 \pm 0.14$ & $10.68 \pm 0.15$ & $16.8 \pm 1.1$ & ST & $1,24,32$ & \\
\hline $1058478-154817 \ldots \ldots \ldots \ldots \ldots .$. & 10949 & DENIS-P J1058-1548 & 14.184 & 0.944 & 1.673 & L3 & $12.99 \pm 0.05$ & $11.32 \pm 0.05$ & $17.33 \pm 0.30$ & Parallax & 13 & 4 \\
\hline $1104012+195921 \ldots \ldots \ldots \ldots \ldots \ldots$. & 10954 & & 14.462 & 0.984 & 1.486 & L4 & $13.09 \pm 0.22$ & $11.61 \pm 0.23$ & $18.8 \pm 2.0$ & ST & 1 & \\
\hline $1108307+683017$ & 10960 & & 13.139 & 0.912 & 1.539 & L0.5 & $11.86 \pm 0.14$ & $10.32 \pm 0.14$ & $18.0 \pm 1.1$ & ST & 7 & \\
\hline $1121492-131308 \mathrm{~A} \ldots \ldots \ldots \ldots \ldots .$. & 10980 & LHS $2397 \mathrm{aA}$ & 11.929 & 0.672 & 1.206 & M8: & $11.17 \pm 0.06$ & $10.01 \pm 0.05$ & $14.45 \pm 0.29$ & Parallax & 3,33 & $2,5,8$ \\
\hline $1121492-131308 \mathrm{~B} \ldots \ldots \ldots \ldots \ldots \ldots$ & $\ldots$ & LHS $2397 \mathrm{aB}$ & $\ldots$ & $\ldots$ & $\ldots$ & L7.5: & $15.00 \pm 0.06$ & $12.78 \pm 0.11$ & $14.45 \pm 0.29$ & Parallax & 34 & $2,5,8$ \\
\hline $1124048+380805$ & 10984 & $\ldots$ & 12.710 & 0.682 & 1.138 & M8.5 & $11.32 \pm 0.15$ & $10.18 \pm 0.16$ & $19.0 \pm 1.4$ & ST & 1 & \\
\hline $1213033-043243 \ldots \ldots \ldots \ldots \ldots \ldots$ & 11044 & $\ldots$ & 14.672 & 0.995 & 1.669 & L5 & $13.55 \pm 0.24$ & $11.89 \pm 0.24$ & $16.7 \pm 1.8$ & ST & 1 & \\
\hline $1224522-123835 \ldots \ldots \ldots \ldots \ldots \ldots \ldots$ & 11068 & BR $1222-1221$ & 12.564 & 0.733 & 1.193 & M9 & $11.40 \pm 0.14$ & $10.21 \pm 0.14$ & $17.06 \pm 1.11$ & Parallax & 19,35 & 5 \\
\hline $1246517+314811 \ldots \ldots \ldots \ldots \ldots \ldots . .$. & 11095 & LHS 2632 & 12.255 & 0.667 & 1.022 & M7.5 & $10.96 \pm 0.21$ & $9.94 \pm 0.21$ & $18.1 \pm 1.8$ & $\mathrm{ST}$ & $3,19,36$ & \\
\hline $1253124+403403$ & 11109 & LHS 2645 & 12.177 & 0.620 & 1.004 & M7.5 & $10.96 \pm 0.21$ & $9.96 \pm 0.22$ & $17.5 \pm 1.7$ & ST & 3,37 & \\
\hline 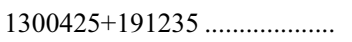 & 11115 & & 12.710 & 0.641 & 1.105 & $\mathrm{~L} 1^{\mathrm{k}}$ & $12.00 \pm 0.14$ & $10.90 \pm 0.15$ & $13.9 \pm 0.9$ & ST & 7 & \\
\hline 1305401-254106А ..................... & 11122 & Kelu-1A & 13.417 & 1.030 & 1.691 & L2: & $12.54 \pm 0.09$ & $10.84 \pm 0.26$ & $18.66 \pm 0.70$ & Parallax & 38 & 4 \\
\hline 1305401-254106B ……............ & $\ldots$ & Kelu-1B & $\ldots$ & $\ldots$ & $\ldots$ & L4: & $13.17 \pm 0.09$ & $11.50 \pm 0.52$ & $18.66 \pm 0.70$ & Parallax & 39,40 & 4 \\
\hline $1309218-233035$...................... & 11127 & CE 303 & 11.769 & 0.682 & 1.103 & M8 & $11.16 \pm 0.18$ & $10.05 \pm 0.18$ & $13.3 \pm 1.1$ & ST & $1,32,41$ & \\
\hline $1332244-044112 \ldots \ldots \ldots \ldots \ldots \ldots$ & 11153 & & 12.342 & 0.591 & 1.046 & M7.5 & $10.96 \pm 0.21$ & $9.92 \pm 0.21$ & $18.9 \pm 1.8$ & ST & 1 & \\
\hline $1356414+434258 \ldots \ldots \ldots \ldots \ldots \ldots . .$. & 11177 & LP 220-13 & 11.704 & 0.673 & 1.070 & M7 & $10.73 \pm 0.25$ & $9.66 \pm 0.25$ & $15.6 \pm 1.8$ & ST & 1,6 & \\
\hline $1403223+300754 \ldots \ldots \ldots \ldots \ldots \ldots . .$. & 11188 & $\ldots$ & 12.691 & 0.683 & 1.065 & M8.5 & $11.32 \pm 0.15$ & $10.25 \pm 0.16$ & $18.8 \pm 1.4$ & ST & 7 & \\
\hline $1411213-211950 \ldots \ldots \ldots \ldots \ldots \ldots . .$. & 11194 & $\ldots$ & 12.442 & 0.619 & 1.122 & M9 & $11.47 \pm 0.14$ & $10.34 \pm 0.14$ & $15.7 \pm 1.0$ & ST & 1 & \\
\hline $1438082+640836 \ldots \ldots \ldots \ldots \ldots \ldots \ldots$ & 11224 & & 12.923 & 0.895 & 1.350 & M9.5 & $11.60 \pm 0.13$ & $10.25 \pm 0.14$ & $18.4 \pm 1.1$ & ST & 1 & \\
\hline $1440229+133923$ & 11230 & LSPM J1440+1339 & 12.379 & 0.635 & 1.088 & M8 & $11.16 \pm 0.18$ & $10.07 \pm 0.18$ & $17.6 \pm 1.5$ & ST & 10,42 & \\
\hline $1456383-280947$...................... & 11264 & LHS 3003 & 9.957 & 0.630 & 1.040 & M7 & $10.83 \pm 0.05$ & $9.79 \pm 0.05$ & $6.37 \pm 0.12$ & Parallax & $3,19,43,44,45$ & 3,5 \\
\hline $1506544+132106 \ldots \ldots \ldots \ldots \ldots \ldots \ldots$ & 11291 & $\ldots$ & 13.414 & 1.002 & 1.666 & L3 & $12.67 \pm 0.20$ & $11.01 \pm 0.20$ & $14.1 \pm 1.3$ & ST & 7 & \\
\hline $1507277-200043$...................... & 11294 & $\ldots$ & 11.718 & 0.668 & 1.064 & M7.5 & $10.96 \pm 0.21$ & $9.90 \pm 0.21$ & $14.2 \pm 1.4$ & ST & 1 & \\
\hline 1507476-162738 …………...... & 11296 & $\ldots$ & 12.822 & 0.920 & 1.520 & L5 & $13.50 \pm 0.03$ & $11.98 \pm 0.03$ & $7.33 \pm 0.03$ & Parallax & 29 & 4 \\
\hline $1515009+484739$ & 11314 & $\ldots$ & 14.060 & 0.991 & 1.495 & L6 & $14.01 \pm 0.30$ & $12.53 \pm 0.30$ & $10.2 \pm 1.4$ & ST & 1,16 & \\
\hline $1521010+505323 \ldots \ldots \ldots \ldots \ldots \ldots . . .$. & 11323 & $\ldots$ & 11.997 & 0.655 & 1.075 & M7.5 & $10.96 \pm 0.21$ & $9.89 \pm 0.21$ & $16.1 \pm 1.6$ & ST & 1 & \\
\hline $1534570-141848 \ldots \ldots \ldots \ldots \ldots$ & 11346 & $\ldots$ & 11.390 & 0.659 & 1.079 & M7 & $10.73 \pm 0.25$ & $9.65 \pm 0.26$ & $13.5 \pm 1.6$ & ST & 10,33 & \\
\hline $1546054+374946 \ldots \ldots \ldots \ldots \ldots \ldots$ & 11439 & $\ldots$ & 12.437 & 0.644 & 1.018 & M7.5 & $10.96 \pm 0.21$ & $9.95 \pm 0.22$ & $19.7 \pm 1.9$ & ST & 7 & \\
\hline $1658037+702701 \ldots \ldots \ldots \ldots \ldots \ldots$ & 11668 & $\ldots$ & 13.309 & 0.766 & 1.390 & L1 & $11.97 \pm 0.04$ & $10.58 \pm 0.04$ & $18.55 \pm 0.24$ & Parallax & 7 & 4 \\
\hline 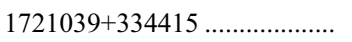 & 11694 & & 13.584 & 0.662 & 1.110 & $\mathrm{~L}^{\mathrm{k}}$ & $12.67 \pm 0.20$ & $11.56 \pm 0.20$ & $15.2 \pm 1.4$ & ST & 1 & \\
\hline 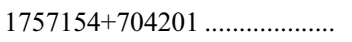 & 11735 & LP $44-162$ & 11.446 & 0.604 & 1.073 & M7.5 & $10.96 \pm 0.21$ & $9.89 \pm 0.21$ & $12.5 \pm 1.2$ & ST & 6,7 & \\
\hline 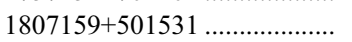 & 11756 & & 12.963 & 0.814 & 1.356 & L1.5 & $12.15 \pm 0.15$ & $10.79 \pm 0.16$ & $14.6 \pm 1.1$ & ST & 1,16 & \\
\hline $1835379+325954 \ldots \ldots \ldots \ldots \ldots \ldots \ldots$ & 11792 & LSR J1835+3259 & 10.273 & 0.691 & 1.119 & M8.5 & $11.51 \pm 0.03$ & $10.39 \pm 0.04$ & $5.67 \pm 0.02$ & Parallax & 46,47 & 9 \\
\hline $1843221+404021^{1} \ldots \ldots \ldots \ldots \ldots \ldots$ & 11800 & LHS 3406 & 11.299 & 0.632 & 1.030 & $\mathrm{M} 8^{1}$ & $10.55 \pm 0.04$ & $9.52 \pm 0.04$ & $14.14 \pm 0.16$ & Parallax & 1,3 & 8 \\
\hline
\end{tabular}


TABLE 9-Continued

\begin{tabular}{|c|c|c|c|c|c|c|c|c|c|c|c|c|}
\hline \multirow[b]{2}{*}{ 2MASSI Designation ${ }^{a}$} & \multirow[b]{2}{*}{ 2MUCD No. } & \multirow[b]{2}{*}{ Other NAMES } & \multicolumn{3}{|c|}{ 2MASS } & \multirow{2}{*}{$\begin{array}{c}\text { Optical } \\
\text { Spectral Type }\end{array}$} & \multirow[b]{2}{*}{$M_{J}$} & \multirow[b]{2}{*}{$M_{K_{S}}$} & \multirow{2}{*}{$\begin{array}{c}d \\
(\mathrm{pc})^{\mathrm{c}}\end{array}$} & \multirow{2}{*}{$\begin{array}{c}d \\
\text { SoURCE }^{\mathrm{c}}\end{array}$} & \multirow{2}{*}{$\begin{array}{l}\text { Discovery } \\
\text { Refs. }\end{array}$} & \multirow{2}{*}{$\begin{array}{l}\text { Parallax } \\
\text { Refs. }\end{array}$} \\
\hline & & & $J$ & $J-H$ & $J-K_{S}$ & & & & & & & \\
\hline $2002507-052152$. & 11946 & $\ldots$ & 15.320 & 1.093 & 1.959 & L6 & $14.02 \pm 0.30$ & $12.06 \pm 0.31$ & $18.2 \pm 2.6$ & ST & 10 & \\
\hline 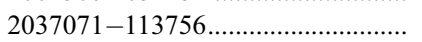 & 12027 & $\ldots$ & 12.284 & 0.665 & 1.024 & M8 & $11.16 \pm 0.18$ & $10.13 \pm 0.18$ & $16.8 \pm 1.4$ & ST & 1 & \\
\hline $2057540-025230$ & 12054 & $\ldots$ & 13.123 & 0.850 & 1.375 & L1.5 & $12.15 \pm 0.15$ & $10.77 \pm 0.16$ & $15.7 \pm 1.1$ & ST & 1 & \\
\hline $2104149-103736$ & 12059 & $\ldots$ & 13.846 & 0.887 & 1.491 & L2.5 & $12.48 \pm 0.18$ & $10.99 \pm 0.19$ & $18.7 \pm 1.6$ & ST & 1,18 & \\
\hline $2224438-015852$ & 12128 & - & 14.052 & 1.249 & 2.035 & L4.5 & $13.75 \pm 0.04$ & $11.72 \pm 0.04$ & $11.35 \pm 0.14$ & Parallax & 29 & 4,6 \\
\hline $2237325+392239$ & 12145 & G216-7B & 13.346 & 0.664 & 1.192 & M9.5 & $11.96 \pm 0.09$ & $10.77 \pm 0.09$ & $18.89 \pm 0.69$ & Parallax & 48 & 10 \\
\hline 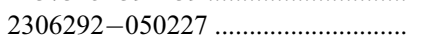 & 12171 & $\ldots$ & 11.372 & 0.654 & 1.084 & M8 & $11.16 \pm 0.18$ & $10.07 \pm 0.18$ & $11.0 \pm 0.9$ & ST & 7 & \\
\hline 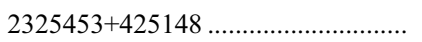 & 13227 & $\ldots$ & 15.512 & 1.052 & 1.705 & L8 & $14.77 \pm 0.30$ & $13.06 \pm 0.31$ & $14.1 \pm 2.0$ & ST & 10 & \\
\hline $2349489+122438 \ldots \ldots \ldots \ldots \ldots \ldots \ldots \ldots \ldots$ & 12217 & LP 523-55 & 12.615 & 0.663 & 1.053 & M8 & $11.16 \pm 0.18$ & $10.10 \pm 0.18$ & $19.6 \pm 1.6$ & ST & 6,7 & \\
\hline $2351504-253736$ & 12220 & $\ldots$ & 12.458 & 0.731 & 1.170 & M8 & $11.16 \pm 0.18$ & $9.99 \pm 0.18$ & $18.2 \pm 1.5$ & ST & 10 & \\
\hline
\end{tabular}

NotE.-Table 9 is also available in machine-readable form in the electronic edition of the Astronomical Journal.

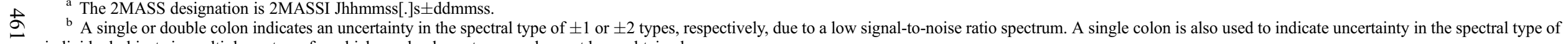
individual objects in multiple systems for which resolved spectroscopy has not been obtained.

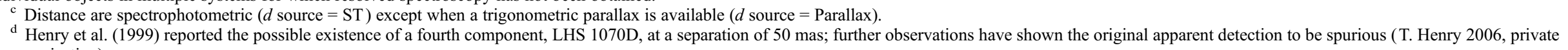
communication).
${ }^{\mathrm{e}}$ Bouy et al. (2005) reported a $\mathrm{T}$ dwarf as a possible third component to the system. The $\mathrm{T}$ dwarf is not included in the luminosity function estimate.

e Bouy et al. (2005) reported a T dwarf as a possible third component to the system. The
${ }^{f}$ Variable and/or flaring object that will be discussed in detail in Schmidt et al. (2006).

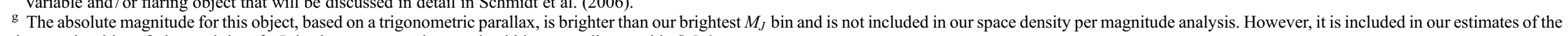
total space densities of ultracool dwarfs. It is also a suspected unresolved binary, as discussed in $\S 5.6$.

${ }^{h}$ This object has been resolved into an L/T binary by Burgasser et al. (2005b). The T dwarf is not included in the luminosity function estimate.

i This object displays features of low gravity, and a spectral type of M9: was used to estimate $M_{J}$.

${ }_{\mathrm{j}}^{\mathrm{j}}$ Change in spectral type from Paper V.

${ }^{\mathrm{k}}$ Suspected low-metallicity dwarf as discussed in $\S 5.4$.

Suspected low-metallicity dwarf as discussed in $\S 5.4$.
Suspected unresolved binary as discussed in $\S 5.6$.

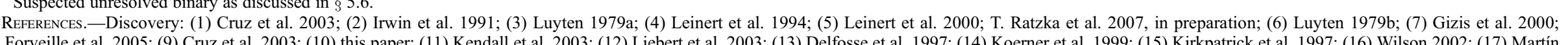

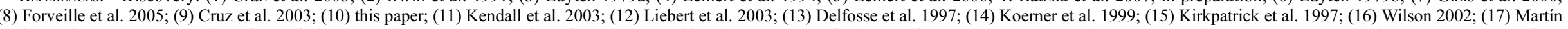

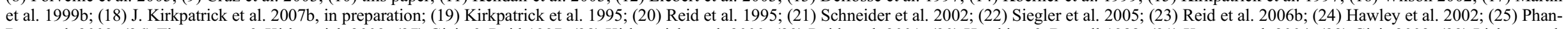

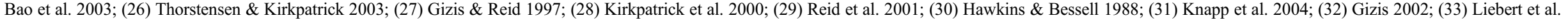

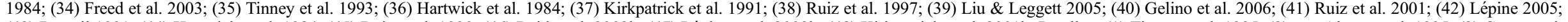

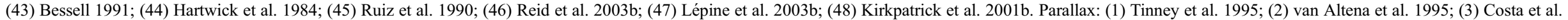
2005; (4) Dahn et al. 2002; (5) Tinney 1996; (6) Vrba et al. 2004; (7) Thorstensen \& Kirkpatrick 2003; (8) Monet et al. 1992; (9) Reid et al. 2003b; (10) Perryman 1997. 
TABLE 10

Objects with Distance Estimates within $1 \sigma$ of 20 pc

\begin{tabular}{|c|c|c|c|c|c|c|c|c|c|c|}
\hline \multirow[b]{2}{*}{ 2MASSI Designation ${ }^{\mathrm{a}}$} & \multirow[b]{2}{*}{ 2MUCD No. } & \multirow[b]{2}{*}{ OTHER NAMES } & \multicolumn{3}{|c|}{ 2MASS } & \multirow[b]{2}{*}{ Optical Spectral Type ${ }^{b}$} & \multirow[b]{2}{*}{$M_{J}$} & \multirow{2}{*}{$\begin{array}{c}d \\
(\mathrm{pc})^{\mathrm{c}}\end{array}$} & \multirow{2}{*}{$\begin{array}{c}d \\
\text { SoURCE }^{\mathrm{c}}\end{array}$} & \multirow[b]{2}{*}{ REFS. } \\
\hline & & & $J$ & $J-H$ & $J-K_{S}$ & & & & & \\
\hline 0050244-153818 ……................. & 10045 & SIPS $0050-1538$ & 13.765 & 0.673 & 1.126 & L1 & $12.00 \pm 0.29$ & $22.5 \pm 3.0$ & ST & 1,2 \\
\hline 0120491-074103 ……................ & 10076 & $\ldots$ & 12.976 & 0.684 & 1.122 & M8 & $11.16 \pm 0.35$ & $23.1 \pm 3.8$ & ST & 3 \\
\hline $0131183+380155 \ldots \ldots \ldots \ldots \ldots \ldots \ldots$ & 13061 & $\ldots$ & 14.694 & 1.022 & 1.666 & L4 & $13.09 \pm 0.44$ & $20.9 \pm 4.3$ & ST & 2 \\
\hline $0240295+283257 \ldots \ldots \ldots \ldots \ldots \ldots \ldots$ & 10138 & $\ldots$ & 12.679 & 0.701 & 1.103 & M7.5 & $10.96 \pm 0.21$ & $22.0 \pm 2.2$ & ST & 4 \\
\hline 0314401-045031 ………............. & 10172 & $\ldots$ & 12.656 & 0.653 & 1.035 & M7.5 & $10.96 \pm 0.21$ & $21.8 \pm 2.1$ & ST & 3 \\
\hline $0428509-225322 \ldots \ldots \ldots \ldots \ldots \ldots \ldots$ & 10286 & $\ldots$ & 13.579 & 0.882 & 1.459 & $\mathrm{~L} 1$ & $12.00 \pm 0.14$ & $20.7 \pm 1.4$ & ST & 5 \\
\hline $0614528+453655 \ldots \ldots \ldots \ldots \ldots \ldots \ldots$ & 10529 & $\ldots$ & 13.003 & 0.755 & 1.234 & M9 & $11.47 \pm 0.14$ & $20.3 \pm 1.3$ & ST & 3 \\
\hline $0810586+142039$ & 10699 & $\ldots$ & 12.714 & 0.669 & 1.104 & M8 & $11.16 \pm 0.18$ & $20.5 \pm 1.7$ & ST & 3 \\
\hline 0850017-192418 …….................. & 10768 & $\ldots$ & 12.816 & 0.682 & 1.189 & M8 & $11.16 \pm 0.18$ & $21.5 \pm 1.8$ & ST & 3 \\
\hline 0952219-192431В ........................ & 10855 & $\ldots$ & 11.877 & 0.595 & 1.031 & M7: & $10.73 \pm 0.50$ & $25.6 \pm 7.2$ & ST & $3,4,6$ \\
\hline $1003191-010507$............................ & 10873 & LHS 5165/DENIS-P J1003-0105 & 12.352 & 0.667 & 1.085 & M7 & $10.73 \pm 0.25$ & $21.1 \pm 2.5$ & ST & $7,8,9$ \\
\hline $1017075+130839$ & 10893 & $\cdots$ & 14.111 & 0.879 & 1.435 & $\mathrm{~L} 2$ & $12.31 \pm 0.34$ & $22.9 \pm 3.6$ & ST & 3 \\
\hline $1019568+732408 \ldots \ldots \ldots \ldots \ldots \ldots \ldots \ldots$ & 10900 & $\cdots$ & 12.884 & 0.637 & 1.100 & M8.5 & $11.32 \pm 0.15$ & $20.6 \pm 1.5$ & ST & 3 \\
\hline $1141440-223215 \ldots \ldots \ldots \ldots \ldots \ldots \ldots$ & 11005 & $\ldots$ & 12.651 & 0.657 & 1.090 & M7.5 & $10.96 \pm 0.21$ & $21.8 \pm 2.1$ & ST & 3 \\
\hline $1152426+243807$......................... & 11018 & $\cdots$ & 13.033 & 0.766 & 1.286 & M9 & $11.47 \pm 0.14$ & $20.6 \pm 1.4$ & ST & 3 \\
\hline 1228152-154734A ....................... & 11073 & DENIS-P J1228.2-1547A & 14.375 & 1.012 & 1.570 & L5: & $13.60 \pm 0.10$ & $20.2 \pm 0.8$ & Parallax & 10,11 \\
\hline $1228152-154734 \mathrm{~B} \ldots \ldots \ldots \ldots \ldots \ldots \ldots$ & 11073 & DENIS-P J1228.2-1547B & & & & L5: & $13.60 \pm 0.10$ & $20.2 \pm 0.8$ & Parallax & 12,13 \\
\hline $1326298-003831 \ldots \ldots \ldots \ldots \ldots \ldots \ldots$ & 11143 & SDSS $1326-0038$ & 16.110 & 1.066 & 1.879 & L8: & $14.60 \pm 0.29$ & $20.0 \pm 2.5$ & Parallax & $14,15,16$ \\
\hline $1448033+155414 \ldots \ldots \ldots \ldots \ldots \ldots \ldots \ldots$ & 11248 & LHS 2980 & 12.481 & 0.629 & 1.010 & M7 & $10.73 \pm 0.25$ & $22.4 \pm 2.6$ & ST & 17 \\
\hline $1553199+140033$........................... & 11462 & $\cdots$ & 13.019 & 0.750 & 1.172 & M9 & $11.47 \pm 0.14$ & $20.5 \pm 1.3$ & ST & 4 \\
\hline 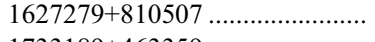 & 11595 & $\cdots$ & 13.042 & 0.709 & 1.168 & M9 & $11.47 \pm 0.14$ & $20.7 \pm 1.4$ & ST & 4 \\
\hline $1733189+463359 \ldots \ldots \ldots \ldots \ldots \ldots \ldots \ldots$ & 11712 & $\cdots$ & 13.214 & 0.806 & 1.357 & M9.5 & $11.60 \pm 0.13$ & $21.0 \pm 1.3$ & ST & 4 \\
\hline 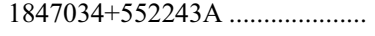 & 11803 & $\ldots$ & 11.919 & 0.641 & 1.021 & M7: & $10.73 \pm 0.50$ & $23.2 \pm 5.4$ & ST & 3 \\
\hline 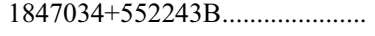 & 11803 & $\ldots$ & 11.919 & 0.641 & 1.021 & M7.5: & $10.96 \pm 0.42$ & $23.2 \pm 5.4$ & ST & 18 \\
\hline 2014035-201621 ……............... & 11977 & $\ldots$ & 12.527 & 0.664 & 1.092 & M7.5 & $10.96 \pm 0.21$ & $20.5 \pm 2.0$ & ST & 3 \\
\hline $2132114+134158 \ldots \ldots \ldots \ldots \ldots \ldots \ldots$ & 13177 & $\ldots$ & 15.785 & 1.201 & 1.951 & L6 & $14.02 \pm 0.30$ & $22.5 \pm 3.2$ & ST & 2 \\
\hline 2158045-155009 …………........ & 12101 & $\ldots$ & 14.949 & 1.033 & 1.801 & L4: & $13.09 \pm 0.44$ & $23.5 \pm 4.8$ & ST & 2,19 \\
\hline $2234139+235955 \ldots \ldots \ldots \ldots \ldots \ldots \ldots \ldots$ & 12139 & $\cdots$ & 13.176 & 0.823 & 1.341 & M9.5 & $11.60 \pm 0.13$ & $20.7 \pm 1.3$ & ST & 4 \\
\hline $2235490+184029$ & 12142 & LP 460-44 & 12.458 & 0.632 & 1.125 & M7 & $10.73 \pm 0.25$ & $22.1 \pm 2.6$ & ST & 4,20 \\
\hline 2331016-040619А........................ & 12191 & $\cdots$ & 12.937 & 0.648 & 1.007 & M8: & $11.16 \pm 0.35$ & $23.3 \pm 3.8$ & ST & 4 \\
\hline 2331016-040619В ……................ & 12191 & $\cdots$ & $\cdots$ & $\cdots$ & $\cdots$ & L7: & $14.45 \pm 0.50$ & $23.3 \pm 3.8$ & ST & 21,22 \\
\hline $2334394+193304$ & 12194 & $\cdots$ & 12.769 & 0.695 & 1.128 & M8 & $11.16 \pm 0.18$ & $21.0 \pm 1.8$ & ST & 4 \\
\hline $2344062-073328$ & 12212 & $\ldots$ & 14.850 & 0.985 & 1.621 & L4.5 & $13.32 \pm 0.23$ & $20.2 \pm 2.2$ & ST & 2 \\
\hline
\end{tabular}

Noте.-Table 10 is also available in machine-readable form in the electronic edition of the Astronomical Journal.

a The 2MASS designation is 2MASSI Jhhmmss[.]s \pm ddmmss.

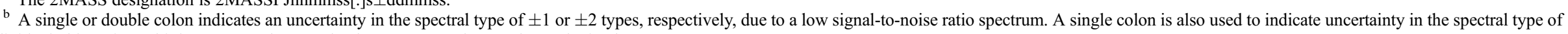
individual objects in multiple systems when resolved spectroscopy has not been obtained.

c Distance are spectrophotometric $(d$ source $=\mathrm{ST})$ except when a trigonometric parallax is available $(d$ source $=$ Parallax $)$.

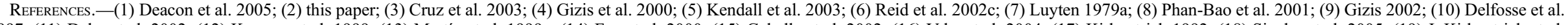

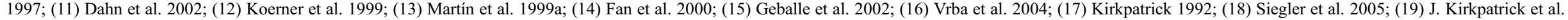
2007b, in preparation; (20) Luyten 1979b; (21) Close et al. 2002; (22) Close et al. 2003. 

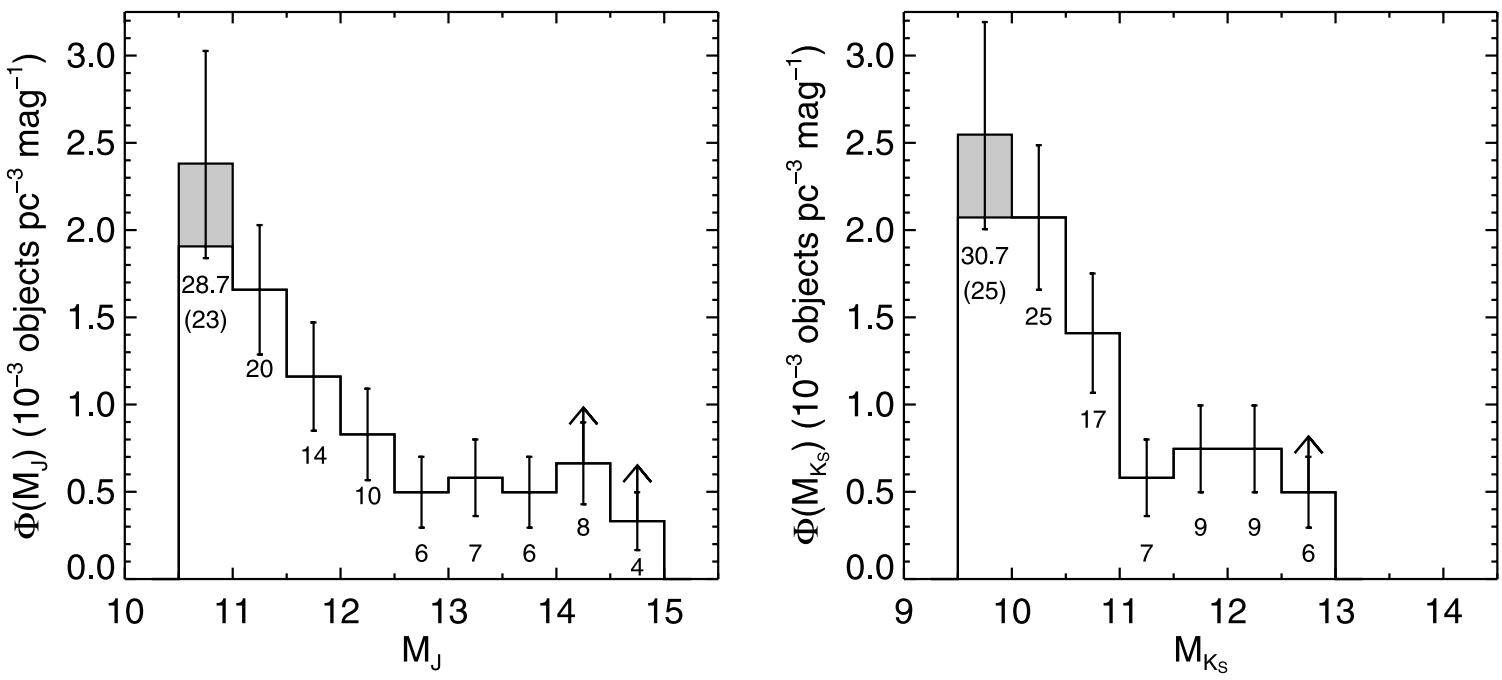

FIG. 16.-Malmquist-corrected near-infrared luminosity function with Poisson uncertainties (unshaded) and the correction for missing M7 dwarfs (shaded; see § 6.2.2). Each bin is labeled with the number of objects used to calculate the space density. The space densities are listed in Table 11. Our measurement is a lower limit for $14<M_{J}<15$ and $12.5<M_{K_{S}}<13$ because the 2MU2 sample is incomplete for L7 and L8 dwarfs and does not include early- and mid-T dwarfs.

we derive uncertainties of $0.13 \mathrm{mag}<\sigma<0.42 \mathrm{mag}$ resulting in corrections of 0.02 to 0.24 mag. These corrections are applied to our measured $\Phi\left(M_{J}\right)$ and $\Phi\left(M_{K_{S}}\right)$.

\subsection{Unresolved Binary Systems}

Unrecognized binarity is likely to affect the statistical properties of the $20 \mathrm{pc} 2 \mathrm{MU} 2$ sample. The sample includes members of 10 known multiple systems. Eight of those systems have trigonometric parallax data. Four are systems in which one component falls beyond the spectral-type limits of the present sample. Those systems are LHS 1070 (2M 0024-27), in which the primary is an M5.5 dwarf, G216-7 (2M 2237+29), in which G216-7A is an M0 dwarf, SDSS J042348.57-041403.5, in which the secondary is an early-type T dwarf (Burgasser et al. 2005b), and DENIS-P J020529.0-115925, in which a possible T-type component has been reported (Bouy et al. 2005).

More than half of the sources in the $20 \mathrm{pc} 2 \mathrm{MU} 2$ sample have either high spatial resolution ground-based observations (Koerner et al. 1999; Close et al. 2003; Siegler et al. 2005) or imaging by the Hubble Space Telescope (Reid et al. 2001, 2006a; Gizis et al. 2003). Of the 55 systems that have been targeted, nine are resolved as close binaries, yielding an observed binary fraction of $17_{-4}^{+6 \%}$, consistent with prior analysis (Gizis et al. 2003; Bouy et al. 2003; Burgasser et al. 2006b). (See Reid et al. [2006a] for a thorough discussion of the binary frequency of our $20 \mathrm{pc}$ sample.) Applying this binary fraction to the 36 unobserved objects suggests that there are approximately five currently unresolved binaries included in our measurement of $\Phi\left(M_{J}\right)$ and $\Phi\left(M_{K_{S}}\right)$. Indeed, as discussed in $\S 5.6$, we already suspect two objects to be unresolved binaries based on their overluminosity.

Burgasser (2004b) has modeled multiplicity corrections for a wide range of scenarios for a magnitude-limited observational sample. The vast majority (34/36) of sources that lack high-resolution imaging also lack trigonometric parallax data. As a result, these sources are effectively a magnitude-limited sample, and Burgasser's analysis is appropriate. In the most extreme case of a binary fraction of $50 \%$, the correction is only $20 \%$ to the late- $\mathrm{L}$ dwarfs. Under more likely circumstances ( binary fraction $\sim 20 \%$ ), the space density of late-M dwarfs is overestimated by $5 \%$ while the density of late- $\mathrm{L}$ dwarfs is underestimated by about $8 \%$. We also note that $\sim 5 \%$ of the sources in our sample may prove to be unrecognized spectroscopic ( $\Delta<2 \mathrm{AU}$ ) binaries (Reid et al. 2002c; Basri \& Reiners 2006). Nonetheless, we expect the effects on $\Phi\left(M_{J}\right)$ and $\Phi\left(M_{K_{S}}\right)$ due to unrecognized binarity to be small $(<10 \%)$ and we do not apply any corrections for them.

\subsection{The Luminosity Function}

We show the Malmquist-corrected $J$ - and $K_{S}$-band luminosity functions derived from the $20 \mathrm{pc} 2 \mathrm{MU} 2$ sample in Figure 16 and list the measured space densities in Table 11. As outlined earlier in this section ( $\S 6.2 .2$ ), the color-magnitude criteria used to define the sample led to incomplete sampling at spectral types $\mathrm{M} 7 / \mathrm{M} 8$ and L7/L8. We have made an explicit allowance for the

TABLE 11

The 20 pe Ultracool Dwarf $J$ - and $K_{S}$-Band Luminosity Functions

\begin{tabular}{|c|c|c|}
\hline Magnitude & $N_{\text {tot }}$ & $\begin{array}{c}\rho_{\text {tot }} \\
\left(10^{-3} \text { dwarfs } \mathrm{pc}^{-3}\right)\end{array}$ \\
\hline \multicolumn{3}{|c|}{$J$ Band } \\
\hline $10.75 \ldots \ldots \ldots$ & $28.7^{\mathrm{a}}$ & $2.38 \pm 0.64^{\mathrm{a}}$ \\
\hline 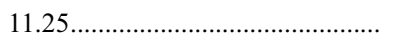 & 20 & $1.66 \pm 0.37$ \\
\hline $11.75 \ldots \ldots \ldots \ldots \ldots \ldots \ldots \ldots \ldots \ldots . .$. & 14 & $1.16 \pm 0.31$ \\
\hline 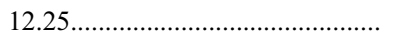 & 10 & $0.83 \pm 0.26$ \\
\hline $12.75 \ldots \ldots \ldots \ldots \ldots$ & 6 & $0.50 \pm 0.20$ \\
\hline 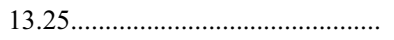 & 7 & $0.58 \pm 0.22$ \\
\hline 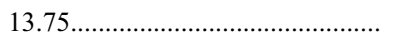 & 6 & $0.50 \pm 0.20$ \\
\hline 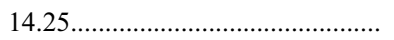 & 8 & $>0.66 \pm 0.23$ \\
\hline 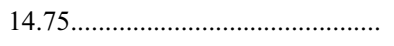 & 4 & $>0.33 \pm 0.17$ \\
\hline \multicolumn{3}{|c|}{$K_{S}$ Band } \\
\hline 9.75 & $30.7^{\mathrm{a}}$ & $2.55 \pm 0.41^{\mathrm{a}}$ \\
\hline $10.25 \ldots \ldots \ldots \ldots \ldots \ldots \ldots \ldots \ldots \ldots \ldots \ldots . .$. & 25 & $2.07 \pm 0.41$ \\
\hline 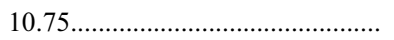 & 17 & $1.41 \pm 0.34$ \\
\hline 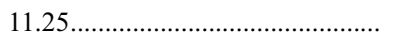 & 7 & $0.58 \pm 0.22$ \\
\hline $11.75 \ldots \ldots \ldots \ldots$ & 9 & $0.75 \pm 0.25$ \\
\hline $12.25 \ldots \ldots \ldots \ldots \ldots \ldots$ & 9 & $0.75 \pm 0.25$ \\
\hline 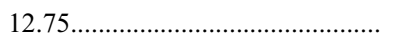 & 6 & $>0.50 \pm 0.20$ \\
\hline
\end{tabular}

Notes.-Values for the space densities plotted in Fig. 16. The absolute magnitudes listed are the center of a 0.5 mag wide bin.

${ }^{\text {a }}$ Corrected value as discussed in $\S$ 6.2.2. 


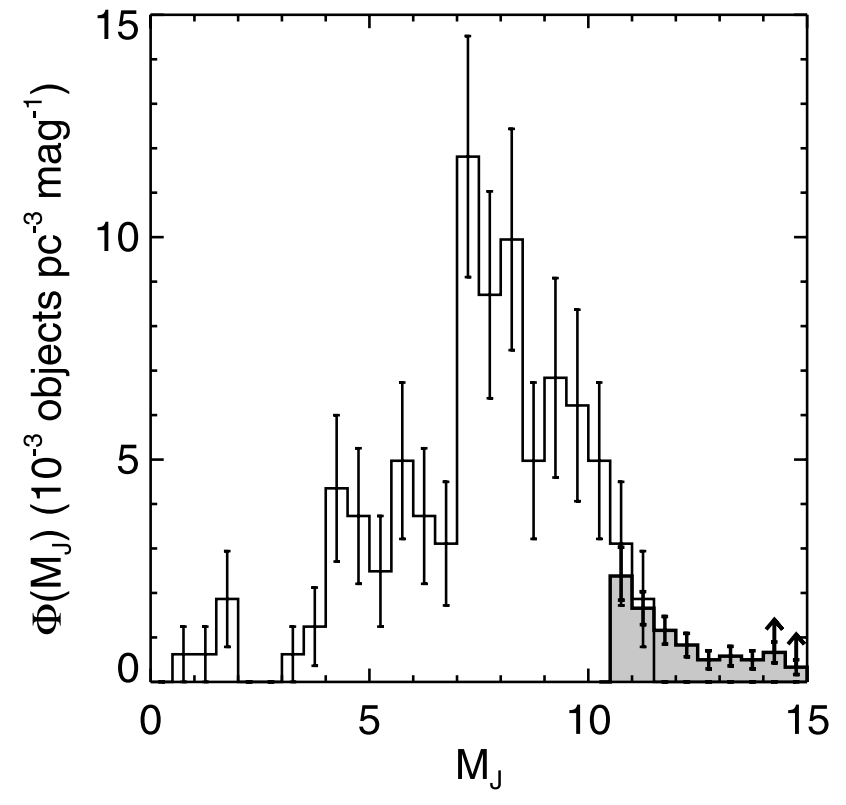

FIG. 17. - J-band luminosity function of the $8 \mathrm{pc}$ (Reid et al. 2003a; unshaded) and $20 \mathrm{pc} 2 \mathrm{MU} 2$ (shaded) samples. The plotted densities are listed in Tables 11 and 12 .

incompleteness at $\mathrm{M} 7$, increasing the space density at $M_{J}=$ $10.75 \pm 0.25$ by $41 \%$.

The correction factors are more difficult to assess at the latest spectral types, however. Due to the incompleteness at late-L types, we take our measured $\Phi\left(M_{K_{S}}\right)$ as a lower limit in the faintest magnitude bin $\left(12.5<M_{K_{S}}<13\right)$. In addition to late-L dwarfs, $\mathrm{T}$ dwarfs contribute to the faintest two bins $\left(14<M_{J}<15\right)$ of $\Phi\left(M_{J}\right)$. Trigonometric parallax results show that brown dwarfs brighten from $M_{J} \sim 15$ at spectral type T0 to $M_{J} \sim 14.5$ at type T3/ T4 (Dahn et al. 2002; Tinney et al. 2003; Vrba et al. 2004). This behavior is probably related to the clearing of dust clouds within the atmosphere, allowing the $\tau=1$ photospheric level to descend to greater physical depths (and higher temperatures) at wavelengths near $1.2 \mu \mathrm{m}$ (Burgasser et al. 2002; Knapp et al. 2004). In any case, our survey was not designed to identify field $\mathrm{T}$ dwarfs, and our measured space densities must represent a lower limit to $\Phi\left(M_{J}\right)$ at $M_{J}>14$.

\section{DISCUSSION}

Integrating our results for the luminosity function, we derive a space density of $(8.7 \pm 0.8) \times 10^{-3} \mathrm{pc}^{-3}$ for M7-L8 dwarfs. Ultracool M dwarfs, spectral types M7 to M9.5, contribute a density of $(4.9 \pm 0.6) \times 10^{-3} \mathrm{pc}^{-3}$. We find the space density of $\mathrm{L}$ dwarfs to be $\geq(3.8 \pm 0.6) \times 10^{-3} \mathrm{pc}^{-3}$, with L0-L3 dwarfs contributing $(1.7 \pm 0.4) \times 10^{-3} \mathrm{pc}^{-3}$ and late-type $\mathrm{L}$ dwarfs responsible for at least $(2.2 \pm 0.4) \times 10^{-3} \mathrm{pc}^{-3}$. Our results are in good agreement with the space density of $4.5 \times 10^{-3} \mathrm{pc}^{-3}$ derived for ultracool M dwarfs by Gizis et al. (2000) but exceed by almost a factor of 2 their initial estimate of the local L dwarf density. Since we are using a significantly larger sample than Gizis et al., our measured densities are likely more accurate.

Putting these results in a wider context, Figure 17 superimposes $\Phi\left(M_{J}\right)$ as derived from our ultracool sample against results for the $8 \mathrm{pc}$ sample (Reid et al. 2003a). As discussed in Reid et al. (2004) $95 \%$ of the 8 pc stars have reliable trigonometric parallaxes; the $J$-band photometry is taken either from $2 \mathrm{MASS}$ or, for bright stars, drawn from the literature and transformed, if
TABLE 12

The 8 pc $J$-Band Luminosity Function

\begin{tabular}{|c|c|c|c|c|}
\hline $\begin{array}{l}M_{J} \\
\text { (1) }\end{array}$ & $\begin{array}{c}N_{\text {sys }} \\
(2)\end{array}$ & $\begin{array}{c}N_{\mathrm{sec}} \\
\text { (3) }\end{array}$ & $\begin{array}{c}\rho_{\text {sys }} \\
\left(10^{-3} \text { dwarfs pc }{ }^{-3}\right) \\
\text { (4) }\end{array}$ & $\begin{array}{c}\rho_{\text {tot }} \\
\left(10^{-3} \text { dwarfs pc } \mathrm{pc}^{-3}\right) \\
(5)\end{array}$ \\
\hline 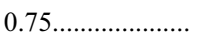 & 1 & 0 & 0.62 & 0.62 \\
\hline $1.25 \ldots \ldots \ldots \ldots \ldots \ldots$ & 1 & 0 & 0.62 & 0.62 \\
\hline $1.75 \ldots \ldots \ldots \ldots \ldots \ldots$ & 3 & 0 & 1.87 & 1.87 \\
\hline 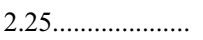 & 0 & 0 & $\ldots$ & $\ldots$ \\
\hline $2.75 \ldots \ldots \ldots \ldots \ldots \ldots \ldots$ & 0 & 0 & $\ldots$ & $\ldots$ \\
\hline $3.25 \ldots \ldots \ldots \ldots \ldots \ldots$ & 1 & 0 & 0.62 & 0.62 \\
\hline $3.75 \ldots \ldots \ldots \ldots \ldots \ldots$ & 2 & 0 & 1.24 & 1.24 \\
\hline 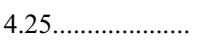 & 6 & 1 & 3.73 & 4.35 \\
\hline 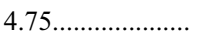 & 6 & 0 & 3.73 & 3.73 \\
\hline 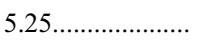 & 3 & 1 & 1.87 & 2.49 \\
\hline $5.75 \ldots \ldots \ldots \ldots \ldots \ldots$ & 3 & 5 & 1.87 & 4.97 \\
\hline 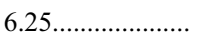 & 6 & 0 & 3.73 & 3.73 \\
\hline 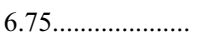 & 5 & 0 & 3.11 & 3.11 \\
\hline $7.25 \ldots \ldots \ldots \ldots \ldots \ldots$ & 15 & 4 & 9.33 & 11.81 \\
\hline 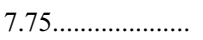 & 10 & 4 & 6.22 & 8.70 \\
\hline 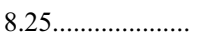 & 12 & 4 & 7.46 & 9.95 \\
\hline 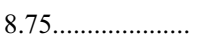 & 6 & 2 & 3.73 & 4.97 \\
\hline 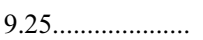 & 11 & 2 & 5.60 & 6.84 \\
\hline 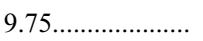 & 6 & 5 & 3.11 & 6.22 \\
\hline $10.25 \ldots \ldots \ldots \ldots \ldots \ldots$ & 3 & 5 & 1.87 & 4.97 \\
\hline 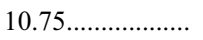 & 3 & 2 & 1.87 & 3.11 \\
\hline $11.25 \ldots \ldots \ldots \ldots \ldots \ldots$ & 1 & 2 & 0.62 & 1.87 \\
\hline
\end{tabular}

Note.-Col. (2) lists the number of systems (single stars and primaries), col. (3) gives the number of secondary components, col. (4) lists the corresponding system space density, and col. (5) lists the total space density, including the contribution from secondaries. The resulting $\Phi\left(M_{J}\right)$ (total space density) is shown in Fig. 17.

necessary, to the CIT system. The derived space densities are listed in Table 12. While the 8 pc sample includes only a handful of ultracool dwarfs, it is clear that the two data sets are in excellent agreement, with the new data confirming the sharp decline in number densities at $M_{J}>10$. In order to get an idea of the space densities in the $I$ band, $M_{I} \sim 13$ for $\mathrm{M} 7$, and $(I-J)$ ranges from 2.4 to 4 for M7-L8 (Dahn et al. 2002).

The overall morphology of the $J$-band luminosity function reflects the convolution of the underlying mass function, $\Psi(M)$, and the $M_{J}$-mass relation. Qualitatively, $\Phi\left(M_{J}\right)$ increases for $0<$ $M_{J}<7$ since the mass function increases with decreasing mass. At fainter magnitudes, $\Phi\left(M_{J}\right)$ turns over not because $\Psi(M)$ changes drastically, but because the slope of the $M_{J}$-mass relation changes; while $\left(\delta\right.$ mass $\left./ \delta M_{J}\right) \sim 0.4 M_{\odot} \mathrm{mag}^{-1}$ for $M_{J}<7$, $\delta$ mass $/ \delta M_{J} \sim 0.07 M_{\odot} \mathrm{mag}^{-1}$ for $7<M_{J}<10$ (Delfosse et al. 2000).

Our analysis shows that $\Phi\left(M_{J}\right)$ declines sharply with decreasing luminosity beyond $M_{J}=10$, reaching a minimum at $M_{J} \sim 13$, roughly corresponding to spectral type L4. Formally, our data indicate that the space densities remain approximately constant at fainter magnitudes; however, since our measurements are lower limits, $\Phi\left(M_{J}\right)$ likely increases at $M_{J}>14$.

Our field survey has uncovered both very low mass stars and substellar mass brown dwarfs from the local population. Assuming a typical age of 2-5 Gyr for a field dwarf, even the most massive brown dwarfs have cooled to L-dwarf temperatures. Moreover, theoretical models (e.g., Burrows et al. 2001) indicate that the hydrogen-burning limit (for solar abundances) corresponds to a temperature of $\sim 1700 \mathrm{~K}$, or spectral type $\sim$ L4 (Golimowski et al. 2004). Thus, early-type L dwarfs include a mix of stars and brown 
dwarfs, with brown dwarfs acquiring increasing dominance from L0 to L4 until they are the sole contributors at $M_{J} \gtrsim 13.5$.

As discussed elsewhere (Chabrier 2003; Burgasser 2004b; Allen et al. 2005), the morphology of the luminosity function is due to this mix of stars and brown dwarfs in the ultracool regime and is qualitatively in accord with theoretical expectations. The drop in number density from M7 to L4 reflects a further contraction in $\delta$ mass $/ \delta M_{J}$. The population of the very lowest mass stars that appear as L dwarfs span an extremely small range in mass $\left(0.075<M_{\odot}<0.085\right)$ and, as a result, are rare. The brown dwarfs in this effective temperature regime are relatively young and are at the high-mass extreme, near the hydrogen-burning limit.

Brown dwarfs dominate the counts beyond the plateau in $\Phi\left(M_{J}\right)$ at $M_{J} \sim 13.5(\sim \mathrm{L} 4)$, and the upturn in number densities reflects the slowdown in cooling rates at lower temperatures. For example, a $0.07 M_{\odot}$ brown dwarf takes $2.7 \mathrm{Gyr}$ to evolve down the $\mathrm{L}$ dwarf sequence, but remains a (cooling) $\mathrm{T}$ dwarf $\left(T_{\mathrm{eff}} \approx\right.$ $1400-600 \mathrm{~K}$ ) for $\sim 30 \mathrm{Gyr}$, or more than two Hubble times; a low-mass, $0.025 M_{\odot}$ brown dwarf spends only $120 \mathrm{Myr}$ as an L dwarf, but 1.5 Gyr as a T dwarf (Burrows et al. 2001).

As noted in $\S 6.5$, early-type $\mathrm{T}$ dwarfs also contribute to the $\Phi\left(M_{J}\right)$ at magnitudes fainter than $M_{J}=14$. Evolutionary models suggest that there may be as many as 80 T0-T5 dwarfs within 20 pc (Burgasser 2004b), as compared with the 12 late-type $\mathrm{L}$ dwarfs contributing to our luminosity function at $M_{J}>14$. Incorporating those cooler dwarfs in the analysis is likely to lead to a steeply increasing $\Phi\left(M_{J}\right)$.

Clearly, one of the aims in deriving the luminosity function for ultracool dwarfs is setting constraints on the mass function, usually parameterized as a power law, $d N / d M \propto M^{-\alpha}$, where the Salpeter value is $\alpha=2.5$. Schultheis et al. (2006) have recently proposed that $\alpha>2$ at low masses (implying that brown dwarfs and low-mass stars essentially account for dark matter). This is in contrast to values of $\alpha \sim 1$ derived in most other analysis, such as Reid et al. (2002a). ${ }^{25}$ A full discussion of this issue is beyond the scope of the present paper. However, we note that the Schultheis et al. analysis rests on matching models against the $V$-band luminosity function for nearby stars and is therefore very weakly constrained for $0.1 M_{\odot}<M<0.15 M_{\odot}$ and essentially unconstrained at lower masses. Burgasser (2004b) and Allen et al. (2005) have shown that our initial results presented in Paper V (which are in general agreement with the luminosity function derived here) are consistent with a range of values of $\alpha<1.5$. While this upper limit is not fully satisfying, it is in disagreement with the steeply rerising mass function as proposed by Schultheis et al.

\section{SUMMARY}

We have mined the 2MASS Second Incremental Data Release PSC for ultracool dwarfs within 20 pc of the Sun. Extensive spectroscopic follow-up has led to the discovery of $\sim 100 \mathrm{~L}$ dwarfs and

\footnotetext{
25 Note that Schultheis et al. (2006) are incorrect in suggesting that the nearby star sample employed in this analysis has significant incompleteness.
}

200 late-M dwarfs; over 50 of these are within 20 pc (Cruz et al. 2003 and this paper), doubling the local census of ultracool dwarfs. In these data we have also uncovered several wide binaries, a handful of young objects, and two slightly metal-poor L dwarfs. Combining our data with previously known nearby late-type dwarfs, we have used 99 objects in $\Phi\left(M_{J}\right)$ and $\Phi\left(M_{K_{S}}\right)$ systems to estimate the $J$ - and $K_{S}$-band luminosity functions of ultracool dwarfs in the solar neighborhood. This work has provided the first robust estimate of the luminosity function of late-type stars and brown dwarfs. We have measured the density of late-M dwarfs (M7-M9.5) to be $(4.9 \pm 0.6) \times 10^{-3} \mathrm{pc}^{-3}$ and the density of $\mathrm{L}$ dwarfs to be at least $(3.8 \pm 0.6) \times 10^{-3} \mathrm{pc}^{-3}$. Reliable discrimination between different models for the underlying mass function must await observational surveys that probe brown dwarfs at temperatures below $\sim 600 \mathrm{~K}$, likely near the boundary between $\mathrm{T}$ and $\mathrm{Y}$ dwarfs.

We would like to acknowledge the numerous NOAO telescope operators and support staff at Kitt Peak, Cerro Tololo, Cerro Panchon, and Mauna Kea that made this work possible and endured our busy observing program: S. Adams, A. Alvarez, T. Beck, M. Bergmann, R. Carrasco, G. Doppmann, E. Eastburn, A. Fhima, B. Gillespie, P. Gomez, A. Guerra, M. HainautRouelle, H. Halbedel, D. Harmer, K. Labrie, L. Macri, H. Mathis, A. Matulonis, D. Maturana, S. Pizarro, P. Prado, K. Roth, K. Volk, and D. Willmarth. We also thank the NOAO Telescope Allocation committees for their enduring support of this project. We acknowledge Finlay Mungall for observing assistance. K. L. C. is supported by an NSF Astronomy and Astrophysics Postdoctoral Fellowship under AST 04-01418. This research was partially supported by a grant from the NASA/NSF NStars initiative, administered by the Jet Propulsion Laboratory, Pasadena, CA. This publication makes use of data products from the Two Micron All Sky Survey, which is a joint project of the University of Massachusetts and Infrared Processing and Analysis Center, California Institute of Technology, funded by the National Aeronautics and Space Administration and the National Science Foundation; and from the NASA/IPAC Infrared Science Archive, which is operated by the Jet Propulsion Laboratory, California Institute of Technology, under contract with the National Aeronautics and Space Administration. This research is based on observations obtained with the Apache Point Observatory $3.5 \mathrm{~m}$ telescope, which is owned and operated by the Astrophysical Research Consortium. This research is also based on observations obtained at the Gemini Observatory, which is operated by the Association of Universities for Research in Astronomy, Inc., under a cooperative agreement with the NSF on behalf of the Gemini partnership: the National Science Foundation (United States), the Particle Physics and Astronomy Research Council (United Kingdom), the National Research Council (Canada), CONICYT (Chile), the Australian Research Council (Australia), CNPq (Brazil), and CONICET (Argentina). This research has made use of the SIMBAD database, operated at CDS, Strasbourg, France.
Ackerman, A. S., \& Marley, M. S. 2001, ApJ, 556, 872

Allen, P. R., Koerner, D. W., Reid, I. N., \& Trilling, D. E. 2005, ApJ, 625, 385 Basri, G., \& Reiners, A. 2006, AJ, 132, 663

Bessell, M. S. 1991, AJ, 101, 662

Borysow, A., Jorgensen, U. G., \& Zheng, C. 1997, A\&A, 324, 185

Bouy, H., Brandner, W., Martín, E. L., Delfosse, X., Allard, F., \& Basri, G. 2003, AJ, 126, 1526

\section{REFERENCES}

Bouy, H., Martín, E. L., Brandner, W., \& Bouvier, J. 2005, AJ, 129, 511

Burgasser, A. J. 2002, Ph.D. thesis, Caltech

2004a, ApJ, 614, L73

2004b, ApJS, 155, 191

Burgasser, A. J., Cruz, K. L., \& Kirkpatrick, J. D. 2006a, ApJ, submitted

Burgasser, A. J., Kirkpatrick, J. D., Cruz, K. L., Reid, I. N., Leggett, S. K., Liebert, J., Burrows, A., \& Brown, M. E. 2006b, ApJS, 166, 585 
Burgasser, A. J., Kirkpatrick, J. D., Liebert, J., \& Burrows, A. 2003a, ApJ, 594, 510 Burgasser, A. J., Kirkpatrick, J. D., \& Lowrance, P. J. 2005a, AJ, 129, 2849 Burgasser, A. J., Marley, M. S., Ackerman, A. S., Saumon, D., Lodders, K., Dahn, C. C., Harris, H. C., \& Kirkpatrick, J. D. 2002, ApJ, 571, L151

Burgasser, A. J., Reid, I. N., Leggett, S. K., Kirkpatrick, J. D., Liebert, J., \& Burrows, A. 2005b, ApJ, 634, L177

Burgasser, A. J., Reid, I. N., Siegler, N., Close, L., Allen, P., Lowrance, P., \& Gizis, J. 2007, in Planets and Protostars V, ed. B. Reipurth, D. Jewitt, \& K. Keil (Tucson: Univ. Arizona Press), 427

Burgasser, A. J., et al. 2003b, ApJ, 592, 1186

Burrows, A., Hubbard, W. B., Lunine, J. I., \& Liebert, J. 2001, Rev. Mod. Phys., 73, 719

Chabrier, G. 2003, PASP, 115, 763

Chabrier, G., \& Baraffe, I. 2000, ARA\&A, 38, 337

Chabrier, G., Baraffe, I., Allard, F., \& Hauschildt, P. 2000, ApJ, 542, 464

Chiu, K., Fan, X., Leggett, S. K., Golimowski, D. A., Zheng, W., Geballe, T. R., Schneider, D. P., \& Brinkmann, J. 2006, AJ, 131, 2722

Close, L. M., Siegler, N., Freed, M., \& Biller, B. 2003, ApJ, 587, 407

Close, L. M., Siegler, N., Potter, D., Brandner, W., \& Liebert, J. 2002, ApJ, 567, L53

Costa, E., Méndez, R. A., Jao, W.-C., Henry, T. J., Subasavage, J. P., Brown, M. A., Ianna, P. A., \& Bartlett, J. 2005, AJ, 130, 337

Crifo, F., Phan-Bao, N., Delfosse, X., Forveille, T., Guibert, J., Martín, E. L., \& Reylé, C. 2005, A\&A, 441, 653

Cruz, K. L., Burgasser, A. J., Reid, I. N., \& Liebert, J. 2004, ApJ, 604, L61

Cruz, K. L., \& Reid, I. N. 2002, AJ, 123, 2828

Cruz, K. L., Reid, I. N., Liebert, J., Kirkpatrick, J. D., \& Lowrance, P. J. 2003, AJ, 126, 2421 (Paper V)

Cushing, M. C., \& Vacca, W. D. 2006, AJ, 131, 1797

Dahn, C. C., et al. 2002, AJ, 124, 1170

Deacon, N. R., Hambly, N. C., \& Cooke, J. A. 2005, A\&A, 435, 363

Delfosse, X., Forveille, T., Ségransan, D., Beuzit, J.-L., Udry, S., Perrier, C., \& Mayor, M. 2000, A\&A, 364, 217

Delfosse, X., et al. 1997, A\&A, 327, L25

Downes, R., Webbink, R. F., \& Shara, M. M. 1997, PASP, 109, 345

Fan, X., et al. 2000, AJ, 119, 928

Forveille, T., et al. 2005, A\&A, 435, L5

Freed, M., Close, L. M., \& Siegler, N. 2003, ApJ, 584, 453

Garcia, B. 1989, Bull. Cent. Donnees Stellaires, 36, 27

Geballe, T. R., et al. 2002, ApJ, 564, 466

Gelino, C. R., Kulkarni, S. R., \& Stephens, D. C. 2006, PASP, 118, 611

Gelino, C. R., Marley, M. S., Holtzman, J. A., Ackerman, A. S., \& Lodders, K. 2002, ApJ, 577, 433

Gizis, J. E. 2002, ApJ, 575, 484

Gizis, J. E., Monet, D. G., Reid, I. N., Kirkpatrick, J. D., Liebert, J., \& Williams, R. J. 2000, AJ, 120, 1085

Gizis, J. E., \& Reid, I. N. 1997, PASP, 109, 849

Gizis, J. E., Reid, I. N., Knapp, G. R., Liebert, J., Kirkpatrick, J. D., Koerner, D. W., \& Burgasser, A. J. 2003, AJ, 125, 3302

Gizis, J. E., Reid, I. N., \& Monet, D. G. 1999, AJ, 118, 997

Golimowski, D. A., et al. 2004, AJ, 127, 3516

Gorlova, N. I., Meyer, M. R., Rieke, G. H., \& Liebert, J. 2003, ApJ, 593, 1074

Hamuy, M., Suntzeff, N. B., Heathcote, S. R., Walker, A. R., Gigoux, P., \& Phillips, M. M. 1994, PASP, 106, 566

Hartwick, F. D. A., Cowley, A. P., \& Mould, J. R. 1984, ApJ, 286, 269

Hawkins, M. R. S., \& Bessell, M. S. 1988, MNRAS, 234, 177

Hawley, S. L., et al. 2002, AJ, 123, 3409

Henry, T. J., Franz, O. G., Wasserman, L. H., Benedict, G. F., Shelus, P. J., Ianna, P. A., Kirkpatrick, J. D., \& McCarthy, D. W. 1999, ApJ, 512, 864

Høg, E., et al. 2000, A\&A, 355, L27

Hook, I. M., Jørgensen, I., Allington-Smith, J. R., Davies, R. L., Metcalfe, N., Murowinski, R. G., \& Crampton, D. 2004, PASP, 116, 425

Irwin, M., McMahon, R. G., \& Reid, N. 1991, MNRAS, 252, 61P

Kendall, T. R., Mauron, N., Azzopardi, M., \& Gigoyan, K. 2003, A\&A, 403, 929

Kirkpatrick, J. D. 1992, Ph.D. thesis, Univ. Arizona

Kirkpatrick, J. D., Barman, T. S., Burgasser, A. J., McGovern, M. R., McLean, I. S., Tinney, C. G., \& Lowrance, P. J. 2006, ApJ, 639, 1120

Kirkpatrick, J. D., Dahn, C. C., Monet, D. G., Reid, I. N., Gizis, J. E., Liebert, J., \& Burgasser, A. J. 2001a, AJ, 121, 3235

Kirkpatrick, J. D., Henry, T. J., \& Irwin, M. J. 1997, AJ, 113, 1421

Kirkpatrick, J. D., Henry, T. J., \& McCarthy, D. W. 1991, ApJS, 77, 417

Kirkpatrick, J. D., Henry, T. J., \& Simons, D. A. 1995, AJ, 109, 797

Kirkpatrick, J. D., Liebert, J., Cruz, K. L., Gizis, J. E., \& Reid, I. N. 2001b, PASP, 113, 814

Kirkpatrick, J. D., et al. 1999, ApJ, 519, 802 . 2000, AJ, 120, 447

Knapp, G. R., et al. 2004, AJ, 127, 3553
Koerner, D. W., Kirkpatrick, J. D., McElwain, M. W., \& Bonaventura, N. R. 1999, ApJ, 526, L25

Leggett, S. K., et al. 2000, ApJ, 536, L35

Leinert, C., Allard, F., Richichi, A., \& Hauschildt, P. H. 2000, A\&A, 353, 691

Leinert, C., Weitzel, N., Richichi, A., Eckart, A., \& Tacconi-Garman, L. E. 1994, A\&A, 291, L47

Lépine, S. 2005, AJ, 130, 1680

Lépine, S., Rich, R. M., \& Shara, M. M. 2003a, ApJ, 591, L49 2003b, AJ, 125, 1598

Lépine, S., \& Shara, M. M. 2005, AJ, 129, 1483

Liebert, J., Boroson, T. A., \& Giampapa, M. S. 1984, ApJ, 282, 758

Liebert, J., Kirkpatrick, J. D., Cruz, K. L., Reid, I. N., Burgasser, A., Tinney, C. G., \& Gizis, J. E. 2003, AJ, 125, 343

Linsky, J. L. 1969, ApJ, 156, 989

Liu, M. C., \& Leggett, S. K. 2005, ApJ, 634, 616

Liu, M. C., Leggett, S. K., Golimowski, D. A., Chiu, K., Fan, X., Geballe, T. R., Schneider, D. P., \& Brinkmann, J. 2006, ApJ, 647, 1393

Liu, W., Hu, J. Y., Li, Z. Y., \& Cao, L. 1999, ApJS, 122, 257

Lodieu, N., Scholz, R.-D., \& McCaughrean, M. J. 2002, A\&A, 389, L20

Luyten, W. J. 1979a, A Catalogue of Stars with Proper Motions Exceeding 0.5" Annually (2nd ed.; Minneapolis: Univ. Minnesota)

. 1979b, New Luyten Catalog of Stars with Proper Motions Larger than Two Tenths of an Arcsecond (2nd ed.; Minneapolis: Univ. Minnesota)

Maiti, M., Sengupta, S., Parihar, P. S., \& Anupama, G. C. 2005, ApJ, 619, L183

Malmquist, K. G. 1920, Medd. Lunds Astron. Obs. Ser. II, 22

Marley, M. S., Seager, S., Saumon, D., Lodders, K., Ackerman, A. S., Freedman, R. S., \& Fan, X. 2002, ApJ, 568, 335

Martín, E. L., Brandner, W., \& Basri, G. 1999a, Science, 283, 1718

Martín, E. L., Delfosse, X., Basri, G., Goldman, B., Forveille, T., \& Zapatero Osorio, M. R. 1999b, AJ, 118, 2466

Martín, E. L., Rebolo, R., \& Zapatero-Osorio, M. R. 1996, ApJ, 469, 706

Massey, P., \& Gronwall, C. 1990, ApJ, 358, 344

Massey, P., Strobel, K., Barnes, J. V., \& Anderson, E. 1988, ApJ, 328, 315

McGovern, M. R., Kirkpatrick, J. D., McLean, I. S., Burgasser, A. J., Prato, L., \& Lowrance, P. J. 2004, ApJ, 600, 1020

McLean, I. S., McGovern, M. R., Burgasser, A. J., Kirkpatrick, J. D., Prato, L., \& Kim, S. S. 2003, ApJ, 596, 561

Monet, D. G., Dahn, C. C., Vrba, F. J., Harris, H. C., Pier, J. R., Luginbuhl, C. B., \& Ables, H. D. 1992, AJ, 103, 638

Morrison, J. E., Röser, S., McLean, B., Bucciarelli, B., \& Lasker, B. 2001, AJ, 121,1752

Nordström, B., et al. 2004, A\&A, 418, 989

Oke, J. B., \& Gunn, J. E. 1983, ApJ, 266, 713

Perryman, M. A. C., et al. 1997, The Hipparcos and Tycho Catalogues (ESA-SP 1200; Noordwijk: ESA)

Phan-Bao, N., et al. 2001, A\&A, 380, 590

- 2003, A\&A, 401, 959

Pokorny, R. S., Jones, H. R. A., Hambly, N. C., \& Pinfield, D. J. 2004, A\&A, 421, 763

Reid, I. N. 2003, AJ, 126, 2449

Reid, I. N., \& Cruz, K. L. 2002, AJ, 123, 2806

Reid, I. N., \& Gizis, J. E. 2005, PASP, 117, 676

Reid, I. N., Gizis, J. E., \& Hawley, S. L. 2002a, AJ, 124, 2721

Reid, I. N., Gizis, J. E., Kirkpatrick, J. D., \& Koerner, D. W. 2001, AJ, 121, 489

Reid, I. N., Hawley, S. L., \& Gizis, J. E. 1995, AJ, 110, 1838 (PMSU)

Reid, I. N., Kilkenny, D., \& Cruz, K. L. 2002b, AJ, 123, 2822

Reid, I. N., Kirkpatrick, J. D., Liebert, J., Gizis, J. E., Dahn, C. C., \& Monet, D. G. 2002c, AJ, 124, 519

Reid, I. N., Lewitus, E., Allen, P. R., Cruz, K. L., \& Burgasser, A. J. 2006a, AJ, 132,891

Reid, I. N., Lewitus, E., Burgasser, A. J., \& Cruz, K. L. 2006b, ApJ, 639, 1114

Reid, I. N., et al. 1999, ApJ, 521, 613

- 2003a, AJ, 125, 354

2003b, AJ, 126, 3007

2004, AJ, 128, 463

Reiners, A., \& Basri, G. 2006, AJ, 131, 1806

Reylé, C., \& Robin, A. C. 2004, A\&A, 421, 643

Ruiz, M. T., Anguita, C., Maza, J., \& Roth, M. 1990, AJ, 100, 1270

Ruiz, M. T., Leggett, S. K., \& Allard, F. 1997, ApJ, 491, L107

Ruiz, M. T., Wischnjewsky, M., Rojo, P. M., \& Gonzalez, L. E. 2001, ApJS, 133,119

Saumon, D., Bergeron, P., Lunine, J. I., Hubbard, W. B., \& Burrows, A. 1994, ApJ, 424, 333

Schmidt, S. J., Cruz, K. L., Bongiorno, B., Reid, I. N., \& Liebert, J. 2006, AJ, submitted

Schneider, D. P., et al. 2002, AJ, 123, 458

Schultheis, M., Robin, A. C., Reylé, C., McCracken, H. J., Bertin, E., Mellier, Y., \& Le Fèvre, O. 2006, A\&A, 447, 185 
Siegler, N., Close, L. M., Cruz, K. L., Martín, E. L., \& Reid, I. N. 2005, ApJ, 621,1023

Skrutskie, M. F., et al. 2006, AJ, 131, 1163

Thorstensen, J. R., \& Kirkpatrick, J. D. 2003, PASP, 115, 1207

Tinney, C. G. 1996, MNRAS, 281, 644

Tinney, C. G., Burgasser, A. J., \& Kirkpatrick, J. D. 2003, AJ, 126, 975
Tinney, C. G., Mould, J. R., \& Reid, I. N. 1993, AJ, 105, 1045

Tinney, C. G., Reid, I. N., Gizis, J., \& Mould, J. R. 1995, AJ, 110, 3014

van Altena, W. F., Lee, J. T., \& Hoffleit, E. D. 1995, General Catalogue of Trigonometric Parallaxes (4th ed.; New Haven: Yale Univ. Obs.)

Vrba, F. J., et al. 2004, AJ, 127, 2948

Wilson, J. C. 2002, Ph.D. thesis, Cornell Univ. 